THE ASTROPHYSiCal Journal SUPPlement SeRIES, 101:41 -85, 1995 November

(c) 1995. The American Astronomical Society. All rights reserved. Printed in U.S.A.

\title{
A REVISED AND EXTENDED CATALOG OF MAGELLANIC SYSTEM CLUSTERS, ASSOCIATIONS, AND EMISSION NEBULAE. I. SMALL MAGELLANIC CLOUD AND BRIDGE
}

\author{
EDUARDO L. D. BICA ${ }^{1,2}$ AND HENRIQUE R. SCHMITT ${ }^{1,3}$ \\ Departamento de Astronomia, IF-UFRGS, CP 15051, CEP 91501-970, Porto Alegre, RS, Brazil \\ Received 1994 November 7; accepted 1995 May 3
}

\begin{abstract}
A survey of extended objects in the Magellanic System was carried out on the ESO/SERC R and J Sky Survey Atlases. The present work is dedicated to the Small Magellanic Cloud and to the inter-Magellanic Cloud region ("Bridge") totaling 1188 objects, of which 554 are classified as star clusters, 343 are emissionless associations, and 291 are related to emission nebulae. The survey includes cross-identifications among catalogs, and we present 284 new objects. We provide accurate positions, classification, homogeneous sizes, and position angles, as well as information on cluster pairs and hierarchical relation for superimposed objects. Two clumps of extended objects in the Bridge and one at the Small Magellanic Cloud wing tip might be currently forming dwarf spheroidal galaxies.
\end{abstract}

Subject headings: Magellanic Clouds — open clusters and associations: general — surveys

\section{INTRODUCTION}

A unified deep catalog and finding charts of star clusters, stellar associations, and emission nebulae in the Magellanic System are fundamental for systematic future studies of the structure and dynamics of the Clouds and for dating their objects in view of recovering the star formation history. In the 1970s the cataloged objects were mostly contained in the LMC and SMC Atlases (Hodge \& Wright 1967, 1977). Since then, their number has increased enormously; for example, by means of the LMC and SMC catalog of emission nebulae by Davies, Elliot, \& Meaburn (1976), Brück's (1976) catalog of SMC clusters based on the UK Schmidt plates, Hodge's ( 1985) SMC catalog of associations, and Hodge's ( 1986) deep catalog of SMC clusters based on CTIO $4 \mathrm{~m}$ plates of particular regions. Automated techniques are a promising tool for the detection of new objects and have already produced results for LMC clusters in a sector of the UKST Sky Survey field 57 (Bhatia \& MacGillivray 1989), and for associations in the SMC and in the interCloud region (Battinelli 1991; Battinelli \& Demers 1992).

Accurate positions are in general given in new LMC catalogs (e.g., Olszewski et al. 1988; Kontizas et al. 1990), but for the SMC until recently only the ESO catalog (Lauberts 1982) had accurate coordinates. Welch (1991) provided astrometric positions for many SMC objects from the catalogs by Kron (1956), Lindsay (1958), Westerlund \& Glaspey (1971), and Hodge \& Wright (1974). Nevertheless, much is still required: Brück's clusters do not have equatorial coordinates, and the positions of Hodge's (1986) clusters and Henize's (1956) emission nebulae are approximate, whereas those of Davies et al. (1976) refer to the brightest part of the nebulae, which in many objects does not coincide with their geometrical center.

The nomenclature of the objects and their cross-identifica-

\footnotetext{
${ }^{1} \mathrm{CNPq}$ fellow.

${ }^{2}$ bica@ifl.if.ufrgs.br.

${ }^{3}$ Present address: Space Telescope Science Institute, 3700 San Martin Drive, Baltimore, MD 21218; schmitt@stsci.edu.
}

tion and equivalence (or not) in different catalogs are problems that a unified catalog has to face and solve. A unified catalog and charts are also fundamental for future surveys of objects with better scale and/or deeper material, or with automated techniques so that previously cataloged objects do not reappear with new designations.

The present work is an effort to provide cross-identifications, accurate positions, homogeneous classification, sizes, and position angles, as well as remarks on the possible hierarchical and physical connection of objects which are superimposed or are close to one another.

This first part of the catalog is devoted to the SMC and to the inter-Cloud region (the stellar and $\mathrm{H}$ I bridge linking the Clouds; see Irwin, Demers \& Kunkel 1990, Grondin, Demers, $\&$ Kunkel 1992, and references therein). In the course of the present revision, 284 previously noncataloged objects in the SMC and the inter-Magellanic Cloud (the "Bridge") were found. Finding charts including all the objects are also provided. In $\S 2$ we describe the catalog construction and show the results. In $\S 3$ we present the finding charts. In $\S 4$ we discuss the spatial distribution of the different object types and their relation to the $\mathrm{H}$ I distribution and study their size distribution. The conclusions of this work are given in $\S 5$.

\section{CATALOG CONSTRUCTION AND RESULTS}

We show in Table 1 information on the catalogs dealing specifically with nonstellar objects in the Clouds which were revised in the present study and which include the SMC and the Bridge region as far east as $\alpha=3^{\mathrm{h}} 40^{\mathrm{m}}$ (Sky Survey field 31). The table contains acronyms, number of entries, and object types which are basically included in each catalog $(\mathrm{C}, \mathrm{A}$, or $\mathrm{N}$, respectively, for star cluster, association, and emission nebula). The present revision includes the SMC objects in the NGC, IC, and ESO catalogs (Lauberts 1982) and the cluster AM-3 from Madore \& Arp (1979). Some catalogs of bright stars and/or of emission-line stars include some compact star clusters and compact $\mathrm{H}$ II regions; their acronyms were used in 
TABLE 1

SMC AND BRIDGE CATALOGS

\begin{tabular}{llrr}
\hline \multicolumn{1}{c}{ Acronym } & \multicolumn{1}{c}{ Reference } & Entries & types \\
\hline SMC-N & Henize 1956 & 117 & $\mathrm{~N}$ \\
K & Kron 1956 & 69 & $\mathrm{C}$ \\
L & Lindsay 1958 & 116 & $\mathrm{C}$ \\
WG & Westerlund \& Glaspey 1971 & 18 & $\mathrm{C}, \mathrm{A}$ \\
HW & Hodge \& Wright 1974 & 86 & $\mathrm{C}$ \\
SMC-DEM & Davies et al. 1976 & 167 & $\mathrm{~N}$ \\
B & Brück 1976 & 168 & $\mathrm{C}, \mathrm{A}$ \\
SNR & Mathewson et al. 1983 & 5 & $\mathrm{~N}$ \\
SNR & Mathewson et al. 1984 & 6 & $\mathrm{~N}$ \\
H-A & Hodge 1985 & 70 & $\mathrm{~A}$ \\
H86- & Hodge 1986 & 213 & $\mathrm{C}$ \\
SMC-DEM & Meaburn 1986 & 5 & $\mathrm{~N}$ \\
IDK & Irwin et al. 1990 & 5 & $\mathrm{~A}$ \\
B-OB & Battinelli 1991 & 31 & $\mathrm{~A}$ \\
BD & Battinelli \& Demers 1992 & 73 & $\mathrm{~A}$ \\
BS & Bica \& Schmitt (this paper) & 284 & $\mathrm{C}, \mathrm{N}, \mathrm{A}$
\end{tabular}

NOTE.-See $\S 2$ for some additional acronyms for catalogs which are not specific of SMC and Bridge nonstellar objects.

the present study in order not to create new designations ( $\mathrm{S}$ from Henize 1956; L61- from Lindsay 1961; Sk from Sanduleak 1968; MA from Meyssonnier \& Azzopardi 1993). As an example, Sk 157 is a compact star cluster (Heydari-Malayeri, Magain, \& Remy 1989). The MA catalog, which is based on objective-prism spectroscopy, consists mostly of emission-line stars but includes both compact emission nebulae and some large ones as well. All entries in those stellar catalogs and in the extended object catalogs listed in Table 1 were checked in the present study.

The objects were examined and measured on the ESO/ SERC R and J Sky Survey Schmidt films at the Instituto de Física, UFRGS. The $\mathrm{R}$ films are less crowded and allow in most cases the observation of the stellar associations and star clusters embedded in or superimposed on the emission regions. They allow one as well to identify and measure star clusters, associations, and emission nebulae even in the densest parts of the SMC. The blue films $(\mathrm{J})$ are deeper and are useful as an independent check on the appearance of the objects and for measuring the overall extent of the star clusters, in particular of the blue ones. The measurements of coordinates, sizes, and position angles were carried out with a $7 \times$ magnifying lens equipped with a graduated glass providing a length resolution of $0.05 \mathrm{~mm}$ (which converts to less than 4 " on the ESO/SERC Schmidt films), and a precision in position angles of $\pm 5^{\circ}$.

The basic steps in the construction of the catalog were as follows: (i) computer files were created containing the original coordinates, which were precessed to the epoch 1950 when necessary; (ii) the objects were identified on the Schmidt films using all the available information in terms of coordinates, descriptions, identification plates, and finding charts in the original papers and in the SMC Atlas (Hodge \& Wright 1977); (iii) cross-identified equivalent objects from different catalogs were merged into a single catalog line; (iv) transparent overlay charts were generated via computer to the scale of the ESO/ SERC Schmidt films; ( $v$ ) original coordinates were replaced by accurate ones when available in the literature, which together with those of SAO stars were used as reference in order to derive accurate positions for the remaining objects via mea- surements of eventual shifts between the positions on the overlays and on the Schmidt films; (vi) major and minor axes and position angle of the major axis were measured.

The accuracy of the coordinates is primarily dependent on the determination of the object center. For star clusters, this uncertainty is typically $10^{\prime \prime}-15^{\prime \prime}$. For large associations and/or $\mathrm{H}$ II complexes, the determination of the center is less precise, but this becomes irrelevant as compared to their extent. The extent, shape, and position angle of the large objects undoubtedly characterize them on the finding charts $(\S 3)$.

We searched for every entry of the previous catalogs and scanned the plates for new objects. The ESO/SERC fields which were studied in the present work are numbers 13, 28, 29, $30,31,50,51$, and 52 . We only include in the catalog (Table 2) the objects which could be detected on the ESO/SERC Schmidt films as nonstellar (typically larger than 15"). In column (1) we show the Sky Survey field quadrant where the object is best seen, and in column (2) we show the object crossidentification in the different catalogs. We have considered as equivalent objects $\mathrm{H}$ II regions and their embedded stellar associations of comparable extent; some objects were separated in two or more parts, and consequently the designation is complemented with " $n$ " for northern part, "se" for southeastern part, etc. We present 284 new objects ( $24 \%$ of the unified catalog) which are identified by the acronym "BS," ordered approximately in right ascension in two series: BS1 to BS247 and BS248 to BS284. In columns (3) and (4) we give the right ascension and declination, respectively, for the epoch 1950 . In column (5) we indicate the object type. Obvious star clusters and emissionless associations are indicated by $\mathrm{C}$ and $\mathrm{A}$, respectively. This distinction is based primarily on the stellar density, but additional criteria are the magnitude distribution of stars and the occurrence of irregular shape, which characterize associations. The intermediate classes $\mathrm{CA}$ and $\mathrm{AC}$ reflect the fact that some doubt arose in the classification, the first letter suggesting the most probable one. We use "NA" for $\mathrm{H}$ II regions with embedded association, whereas "NC" refers to small H II regions with embedded star clusters and/or high surface brightness compact $\mathrm{H}$ II regions. " $\mathrm{N}$ " is reserved for known supernova remnants (Mathewson et al. 1983, 1984) and for a few objects which are seen as nonstellar in the Schmidt films and are classified as planetary nebulae (Meyssonnier \& Azzopardi 1993). "AN" and "CN" are associations and clusters, respectively, which show some traces of emission. In columns (6) and (7) we show the sizes of the major and minor axes, respectively. In column (8) we give the position angle of the major axis $\left(0^{\circ}=\mathrm{N}, 90^{\circ}=\mathrm{E}\right)$. Finally, in column $(9)$ remarks are given: (i) "mP" and " $\mathrm{mT}$ " indicate member of cluster pair or cluster triplet, respectively. Hatzidimitriou \& Bhatia (1990) list 30 SMC cluster pairs, while the present work contains 40 pairs and two triplets; (ii ) "br*" indicates that a bright star is present; (iii) "att" means attached to; (iv) a hierarchical indication is given for objects embedded in or superimposed on larger ones: "in" suggests a possible physical connection, while "sup" suggests a projection.

We present in Table 3 the entries (ordered in right ascension) from the different extended object catalogs which were not included in Table 2 for the following reasons: stellar appearance, nonexisting, star or stars, asterism, galaxy, suspected galaxy, object duplication in the same catalog, and fi- 
nally, an entry from an emission nebula catalog which is a star cluster not related to emission. We have checked all the corrections suggested in previous catalogs, not always confirming them, and we have found new ones as well. "Not clear" means that we have not been able to confirm the classification of the object on the Schmidt film, which in general applies to a few objects in the H86- catalog that were defined as star clusters in better scale plates. Table 3 contains most of the planetary nebulae known in the SMC because their appearance is stellar on the Schmidt films. Table 3 also contains objects from the $S$, L61-, and MA catalogs which are related to extended objects and/or that we initially suspected to be extended, but turned out to be stellar on the Schmidt films.

The present catalog (Table 2) is not intended to be complete in any sense. The objective was to check and cross-identify the entries in previous catalogs, and to search for new similar objects, in order to create a more complete and homogeneous sample.

\section{FINDING CHARTS}

We present in Figure $1 a$ the overall spatial distribution of the catalog entries (Table 2), and in Figure $1 b$ we present an enlargement of the SMC main body; the codes for the detailed finding charts are indicated. In Figures $2 a-2 s$ we present the finding charts where the objects are labeled with their first designation in Table 2. Each figure shows the right ascension and declination $\left(\alpha_{0}, \delta_{0}\right)$ of the origin of the scale in degrees shown in the axes. North and east are indicated in each figure. The object size is plotted to scale and is oriented according to the position angle from Table 2 . The labels are in general placed rightward (west) of the object; exceptions are in general connected with dashed lines to the respective object for clarity. SAO stars (for reference) are shown as circles.

The accurate positions, orientations, and labels facilitate the identification of the objects on any plate. The charts themselves are useful overlays when copied to a suitable scale on transparencies.

\section{DISCUSSION}

The total number of cataloged extended objects (Table 2) in the SMC and in the Bridge as far east as $3^{\mathrm{h}} 40^{\mathrm{m}}$ is 1188 , of which 554 are star clusters $(\mathrm{C}+\mathrm{CA}+\mathrm{CN}), 343$ are emissionless associations $(A+A C+A N)$, and 291 are related to emission nebulae $(\mathrm{NA}+\mathrm{NC}+\mathrm{N})$. Assuming as the borderline between the SMC and the Bridge $\alpha=2^{\mathrm{h}}$, we obtain 1074 objects in the SMC distributed among clusters:associations:nebulae as 543: 246:285; for the Bridge we obtain a total of 114 objects distributed as 11:97:6. These proportions of object types in the SMC and Bridge possibly reflect different star formation histories, but factors such as lower gas density in the Bridge and a different average mass function may play an important role as well.

The star cluster group spans all ages, except those formed in the last few megayears which were included in the emission nebula group (NC). The emissionless associations basically trace star formation in the range $\sim 6-100 \mathrm{Myr}$, as inferred from their integrated $U B V$ colors when compared to those of star clusters with known age (Bica et al. 1995). Since the emission objects are mostly $\mathrm{H}$ II regions, they basically trace star formation in the last 5-6 Myr (Copetti, Pastoriza, \& Dottori 1985). The supernova remnants (SNRs) from massive progenitors trace as well recent star formation (at least $2 / 3$ of the SNRs in the LMC are clearly related to young population environments; Chu \& Kennicutt 1988). However, the sample certainly contains SNRs from lower mass progenitors and some planetary nebulae, which are objects associated to older stellar populations.

The spatial distributions for the whole object sample (Table 2 ), star clusters, emissionless associations, and objects related to emission are compared in Figures $3 a-3 d$, respectively, to the $\mathrm{H}$ I surface density distribution (Mathewson \& Ford 1984). Including outlying clusters (Fig. $3 b$ ), the extent of the SMC cluster system is $\sim 10^{\circ}(10.6 \mathrm{kpc}$ at a distance of $60 \mathrm{kpc})$, which is comparable to that derived by Irwin (1991) from stellar count isopleths. However, most of the clusters seem to form an elliptical distribution of $\sim 6^{\circ} \times 4^{\circ}$ with major axis in the SW$\mathrm{NE}$ direction, as do the denser parts of the $\mathrm{H}$ I distribution. Only two star clusters $(0.4 \%)$ are projected outside the $5 \times 10^{19}$ atoms $\mathrm{cm}^{-2} \mathrm{H}$ I contour, and the fraction is still very small ( $1.3 \%$ ) for those outside the $20 \times 10^{19}$ atoms cm$~^{-2}$ contour. In Figure $3 c$ only one emissionless association $(0.3 \%)$ lies outside the $40 \times 10^{19}$ atoms $\mathrm{cm}^{-2} \mathrm{H}$ I contour, and only $2.0 \%$ are located outside the $50 \times 10^{19}$ atoms cm $\mathrm{cm}^{-2}$ contour. The spatial distribution of the emission nebulae is more concentrated than that of the emissionless associations, which gives a constraint on the minimum $\mathrm{H}$ I surface density for current star formation. Interestingly, we found on the ESO R film an emission object (BS197; see chart 8 in Figs. $2 o$ and $1 a$ ) which lies well outside the main body of the SMC, near the edge of an isolated $50 \times$ $10^{19}$ atoms $\mathrm{cm}^{-2} \mathrm{H}$ i cloud (Fig. $3 d$ ).

Two clumps of extended objects in the Bridge and one at the SMC wing tip are apparent in Figure $3 a$, where they are labeled as D3, D2, and D1, respectively. Their sizes are $1^{\circ} .3 \times 11^{\circ} .0$ (D3), $1^{\circ} .4 \times 0.8$ (D2), and $2^{\circ} .6 \times 11^{\circ} .2$ (D1). At a distance of $57 \mathrm{kpc}$ (between those of the LMC and SMC), these values expressed in degrees can be read in kiloparsecs. Although the structural parameters of dwarf spheroidals are still a matter of debate, especially for Fornax (Mateo et al. 1991), the clump dimensions are similar to tidal radius estimates for Fornax (2-4 kpc; Hodge 1971, Paltoglou \& Freeman 1987, Eskridge 1988), and for Draco and Ursa Minor ( $\approx 1.1 \mathrm{kpc}$; Lake 1990).

The $\mathrm{H}$ I masses of the clumps can be estimated from $M\left(M_{\odot}\right)=$ $A\langle n\rangle \times 8.0 \times 10^{-15}$, where $A\left(\mathrm{kpc}^{2}\right)$ is the surface area and $\langle n\rangle\left(\right.$ atoms $\left.\mathrm{cm}^{-2}\right)$ is the average $\mathrm{H}$ I surface density in the clump region. We obtained $A=3.0,1.0$, and $1.2 \mathrm{kpc}^{2}$ from the clump sizes, respectively for D1, D2, and D3; we estimated from Figure $3\langle n\rangle \approx 100,80$, and $45 \times 10^{19}$ atoms $\mathrm{cm}^{-2}$, respectively. Finally, we derived $\mathrm{H}$ I masses $M \approx 24.0,6.4$, and $4.3 \times 10^{6}\left(M_{\odot}\right)$ for D1, D2, and D3, respectively. These values are encompassed by mass estimates for dwarf spheroidals: Lake ( 1990 ) found $M=10^{7} M_{\odot}$ for Draco and Ursa Minor, and Pryor \& Kormendy (1990) show that it cannot be a factor 2-3 smaller than this for reasonable models; Mateo et al. (1991) derived $M=6 \times 10^{7}-1 \times 10^{8} M_{\odot}$ for Fornax.

Interesting comparisons can be made of the star cluster content of the clumps and that of the dwarf spheroidals. D1 has 
TABLE 2

REvised AND ExTENDED CATALOG

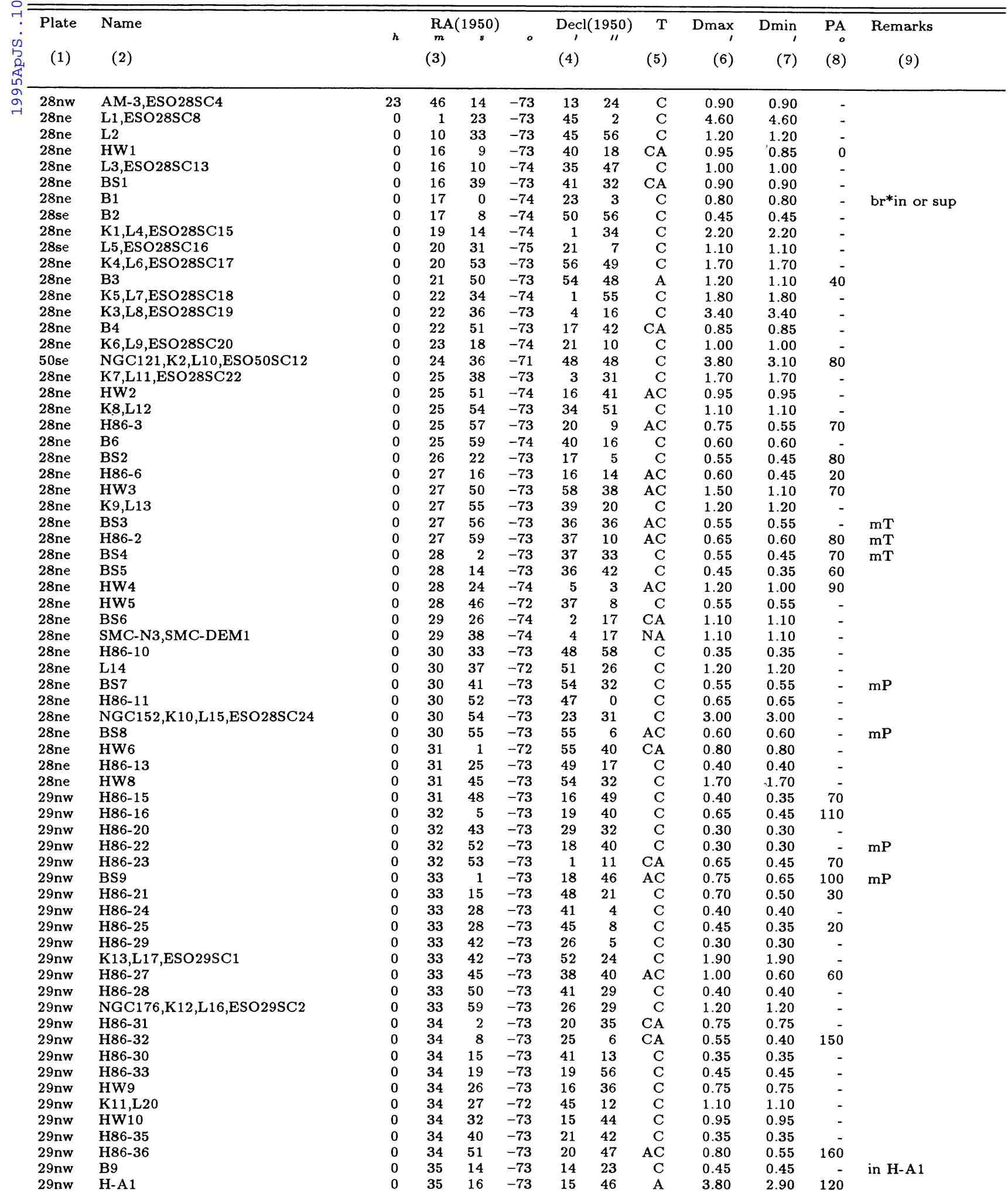


TABLE 2-Continued

\begin{tabular}{|c|c|c|c|c|c|c|c|c|c|c|c|c|}
\hline \multirow{2}{*}{$\begin{array}{l}\text { Plate } \\
\text { (1) }\end{array}$} & \multirow{2}{*}{$\begin{array}{l}\text { Name } \\
\text { (2) }\end{array}$} & \multicolumn{4}{|c|}{ RA(1950) } & \multicolumn{2}{|c|}{$\operatorname{Decl}(1950)$} & \multirow{2}{*}{$\begin{array}{r}\mathrm{T} \\
(5)\end{array}$} & \multirow{2}{*}{$\begin{array}{r}\text { Dmax } \\
(6)\end{array}$} & \multirow{2}{*}{$\begin{array}{r}\text { Dmin } \\
(7)\end{array}$} & PA & Remarks \\
\hline & & $h$ & $\begin{array}{r}m \\
(3)\end{array}$ & & 0 & $\begin{array}{r}\prime \\
(4)\end{array}$ & "I & & & & $\begin{array}{r}0 \\
(8)\end{array}$ & $(9)$ \\
\hline $29 n w$ & K14,L18,SMC-DEM2 & 0 & 35 & 17 & -73 & 16 & 11 & AN & 1.80 & 1.20 & 140 & in $\mathbf{H}-\mathbf{A} 1$ \\
\hline $29 n w$ & $\mathrm{H} 86-38$ & 0 & 35 & 26 & -73 & 18 & 20 & A & 1.20 & 1.20 & - & \\
\hline $29 n w$ & H86-41 & 0 & 35 & 31 & -73 & 14 & 18 & C & 0.55 & 0.40 & 50 & \\
\hline $29 n w$ & HW11 & 0 & 35 & 33 & -73 & 53 & 16 & $\mathrm{C}$ & 1.30 & 1.30 & - & \\
\hline $29 n w$ & BS10 & 0 & 35 & 36 & -73 & 18 & 0 & C & 0.65 & 0.65 & - & \\
\hline $29 n w$ & $\mathrm{H} 86-42$ & 0 & 35 & 43 & -73 & 23 & 46 & C & 0.50 & 0.45 & 80 & \\
\hline $29 n w$ & L19 & 0 & 35 & 45 & -74 & 10 & 57 & C & 1.70 & 1.70 & - & \\
\hline $29 n w$ & B10 & 0 & 35 & 48 & -73 & 29 & 3 & C & 0.80 & 0.80 & - & \\
\hline $29 n w$ & H86-40 & 0 & 35 & 52 & -73 & 45 & 32 & C & 0.45 & 0.35 & 170 & \\
\hline $29 \mathrm{nw}$ & H86-43 & 0 & 35 & 53 & -73 & 23 & 46 & C & 0.55 & 0.55 & - & \\
\hline $29 n w$ & B12 & 0 & 36 & 23 & -73 & 32 & 32 & C & 0.45 & 0.35 & 140 & \\
\hline $29 \mathrm{nw}$ & BS11 & 0 & 36 & 27 & -73 & 39 & 11 & A & 1.20 & 0.50 & 60 & \\
\hline $29 n w$ & B14 & 0 & 36 & 37 & -74 & 5 & 4 & C & 0.60 & 0.45 & 160 & \\
\hline $29 n w$ & H86-47 & 0 & 36 & 52 & -73 & 32 & 46 & $\mathrm{C}$ & 0.30 & 0.30 & - & \\
\hline $29 n w$ & HW12 & 0 & 36 & 55 & -73 & 38 & 56 & $\mathrm{C}$ & 0.80 & 0.80 & - & \\
\hline $29 n w$ & H86-48 & 0 & 36 & 57 & -73 & 40 & 55 & $\mathrm{C}$ & 0.45 & 0.35 & 30 & \\
\hline $29 n w$ & H86-52 & 0 & 37 & 3 & -73 & 16 & 59 & $\mathrm{C}$ & 0.30 & 0.30 & - & \\
\hline $29 n w$ & B16 & 0 & 37 & 4 & -74 & 13 & 18 & C & 0.30 & 0.30 & - & \\
\hline $29 n w$ & BS12 & 0 & 37 & 8 & -74 & 12 & 34 & C & 0.35 & 0.35 & - & \\
\hline $29 n w$ & BS14 & 0 & 37 & 15 & -73 & 31 & 10 & C & 0.55 & 0.55 & - & \\
\hline $29 n w$ & H86-53 & 0 & 37 & 20 & -73 & 10 & 41 & $\mathrm{C}$ & 0.80 & 0.70 & 50 & \\
\hline $29 n w$ & HW12A & 0 & 37 & 28 & -73 & 39 & 33 & $\mathrm{C}$ & 0.55 & 0.45 & 10 & $\mathrm{mP}$ \\
\hline $29 n w$ & H86-55 & 0 & 37 & 30 & -73 & 22 & 52 & C & 0.45 & 0.35 & 80 & \\
\hline $29 n w$ & HW13 & 0 & 37 & 35 & -73 & 42 & 1 & $\mathrm{C}$ & 0.75 & 0.60 & 80 & \\
\hline $29 n w$ & H86-54 & 0 & 37 & 36 & -73 & 39 & 39 & C & 0.55 & 0.55 & - & $\mathrm{mP}$ \\
\hline $29 n w$ & H86-56 & 0 & 37 & 42 & -73 & 12 & 16 & $\mathrm{CA}$ & 1.20 & 0.80 & 140 & \\
\hline $29 n w$ & B15 & 0 & 37 & 46 & -73 & 15 & 7 & $\mathrm{CA}$ & 0.60 & 0.45 & 100 & $\mathrm{mP}$ \\
\hline $29 n w$ & H86-57 & 0 & 37 & 51 & -73 & 15 & 24 & $\mathrm{CA}$ & 0.50 & 0.50 & - & $\mathrm{mP}$ \\
\hline $29 n w$ & H86-58 & 0 & 38 & 10 & -73 & 25 & 2 & C & 0.60 & 0.60 & - & \\
\hline $29 n w$ & BS13 & 0 & 38 & 12 & -73 & 1 & 58 & $\mathrm{CA}$ & 0.90 & 0.75 & 40 & \\
\hline $29 n w$ & $\mathrm{~K} 15, \mathrm{~L} 21$ & 0 & 38 & 16 & -72 & 58 & 24 & C & 1.10 & 1.10 & - & \\
\hline $29 n w$ & BS248 & 0 & 38 & 19 & -73 & 2 & 30 & $\mathrm{AC}$ & 0.65 & 0.50 & 70 & \\
\hline $29 n w$ & HW14 & 0 & 38 & 21 & -74 & 8 & 48 & C & 1.70 & 1.50 & 170 & \\
\hline $29 n w$ & H86-59 & 0 & 38 & 33 & -73 & 44 & 8 & C & 0.50 & 0.45 & 50 & \\
\hline $29 n w$ & NGC220,K18,L22,ESO29SC3 & 0 & 38 & 36 & -73 & 40 & 40 & $\mathrm{C}$ & 1.20 & 1.20 & - & in $\mathrm{H}-\mathbf{A} \mathbf{3}$ \\
\hline $29 n w$ & $\mathrm{~K} 16, \mathrm{~L} 23$ & 0 & 38 & 37 & -73 & 0 & 51 & C & 0.85 & 0.85 & - & \\
\hline $29 n w$ & B25 & 0 & 38 & 38 & -73 & 59 & 50 & $\mathrm{CA}$ & 0.65 & 0.55 & 90 & \\
\hline $29 \mathrm{nw}$ & H86-60 & 0 & 38 & 48 & -73 & 23 & 33 & C & 0.40 & 0.30 & 120 & \\
\hline $29 n w$ & B19 & 0 & 38 & 49 & -73 & 20 & 10 & C & 0.45 & 0.35 & 170 & $\mathrm{mP}$ \\
\hline $29 n w$ & B26 & 0 & 38 & 49 & -74 & 0 & 59 & $\mathrm{C}$ & 0.90 & 0.90 & - & \\
\hline $29 n w$ & B27 & 0 & 38 & 49 & -74 & 8 & 48 & C & 0.40 & 0.40 & - & \\
\hline $29 n w$ & NGC222,K19,L24,ESO29SC4 & 0 & 38 & 50 & -73 & 39 & 33 & C & 1.20 & 1.20 & - & in $\mathbf{H}-\mathbf{A} 3$ \\
\hline $29 n w$ & $\mathrm{H}-\mathrm{A} 3$ & 0 & 38 & 51 & -73 & 39 & 10 & $\mathbf{A}$ & 7.80 & 3.90 & 60 & \\
\hline $29 n w$ & $\mathrm{~B} 20$ & 0 & 38 & 54 & -73 & 20 & 37 & $\mathrm{C}$ & 0.30 & 0.30 & - & $\mathrm{mP}$ \\
\hline $29 n w$ & H86-62 & 0 & 38 & 55 & -73 & 21 & 39 & C & 0.60 & 0.60 & - & \\
\hline $29 n w$ & HW15 & 0 & 38 & 55 & -74 & 17 & 46 & C & 1.00 & 0.75 & 160 & \\
\hline $29 n w$ & B23 & 0 & 39 & 0 & -73 & 40 & 38 & C & 0.60 & 0.60 & - & in $\mathbf{H}-\mathbf{A} \mathbf{3}$ \\
\hline $29 n w$ & $\mathrm{~K} 17, \mathrm{~L} 26$ & 0 & 39 & 5 & -72 & 50 & 47 & C & 1.10 & 1.10 & - & \\
\hline $29 n w$ & SMC-DEM5 & 0 & 39 & 7 & -73 & 52 & 49 & NA & 2.90 & 2.90 & - & \\
\hline $29 n w$ & B18 & 0 & 39 & 11 & -73 & 0 & 0 & C & 0.35 & 0.30 & 120 & \\
\hline $29 n w$ & NGC231,K20,L25,ESO29SC5 & 0 & 39 & 12 & -73 & 37 & 35 & C & 1.80 & 1.80 & - & in $\mathrm{H}-\mathbf{A 3}$ \\
\hline $29 \mathrm{nw}$ & B21 & 0 & 39 & 20 & -73 & 6 & 27 & C & 0.35 & 0.35 & - & $\mathrm{mP}$ \\
\hline $29 n w$ & B22 & 0 & 39 & 26 & -73 & 5 & 59 & $\mathrm{C}$ & 0.35 & 0.35 & - & $\mathrm{mP}$ \\
\hline $29 \mathrm{nw}$ & B24 & 0 & 39 & 26 & -73 & 15 & 18 & $\mathrm{CA}$ & 0.80 & 0.55 & 100 & \\
\hline $29 n w$ & BS15 & 0 & 39 & 27 & -73 & 36 & 58 & A & 2.30 & 2.30 & - & in $\mathbf{H}-\mathbf{A} 3$ \\
\hline $29 \mathrm{nw}$ & $\mathrm{K} 21, \mathrm{~L} 27$ & 0 & 39 & 29 & -73 & 9 & 50 & C & 2.50 & 2.50 & - & \\
\hline $29 \mathrm{nw}$ & B29 & 0 & 40 & 19 & -74 & 0 & 18 & $\mathrm{CA}$ & 0.50 & 0.35 & 100 & $\mathrm{mP}$ \\
\hline $29 n w$ & SMC-DEM6 & 0 & 40 & 20 & -73 & 15 & 52 & NA & 1.10 & 1.10 & - & \\
\hline $29 \mathrm{nw}$ & L61-34,MA37 & 0 & 40 & 22 & -73 & 16 & 20 & $\mathrm{NC}$ & 0.40 & 0.35 & 120 & in SMC-DEMI \\
\hline $29 n w$ & SMC-DEM7 & 0 & 40 & 27 & -74 & 0 & 57 & NA & 1.00 & 0.75 & 10 & \\
\hline $29 n w$ & HW 16 & 0 & 40 & 29 & -74 & 0 & 34 & $\mathrm{CN}$ & 0.60 & 0.60 & - & in SMC-DEM' \\
\hline $29 n w$ & H86-68 & 0 & 40 & 43 & -72 & 43 & 47 & C & 1.10 & 1.10 & - & \\
\hline $29 \mathrm{nw}$ & BS249 & 0 & 40 & 58 & -73 & 26 & 58 & $\mathrm{CA}$ & 0.50 & 0.45 & 60 & in $\mathrm{H}-\mathrm{A} 5$ \\
\hline $29 n w$ & H-A4 & 0 & 41 & 0 & -73 & 34 & 13 & A & 2.10 & 2.10 & - & \\
\hline $29 n w$ & HW18 & 0 & 41 & 4 & -72 & 41 & 6 & C & 1.00 & 1.00 & - & \\
\hline $29 n w$ & L28 & 0 & 41 & 5 & -72 & 51 & 45 & C & 1.00 & 1.00 & - & \\
\hline $29 n w$ & BS 16 & 0 & 41 & 7 & -73 & 26 & 35 & C & 0.55 & 0.50 & 10 & in $\mathrm{H}-\mathbf{A} 5$ \\
\hline $29 n w$ & H-A5 & 0 & 41 & 9 & -73 & 25 & 33 & A & 3.80 & 2.50 & 30 & \\
\hline $29 n w$ & BS18 & 0 & 41 & 9 & -73 & 31 & 54 & A & 1.20 & 1.20 & - & \\
\hline $29 n w$ & B28 & 0 & 41 & 15 & -72 & 31 & 50 & $\mathrm{CA}$ & 0.85 & 0.55 & 80 & \\
\hline
\end{tabular}


TABLE 2-Continued

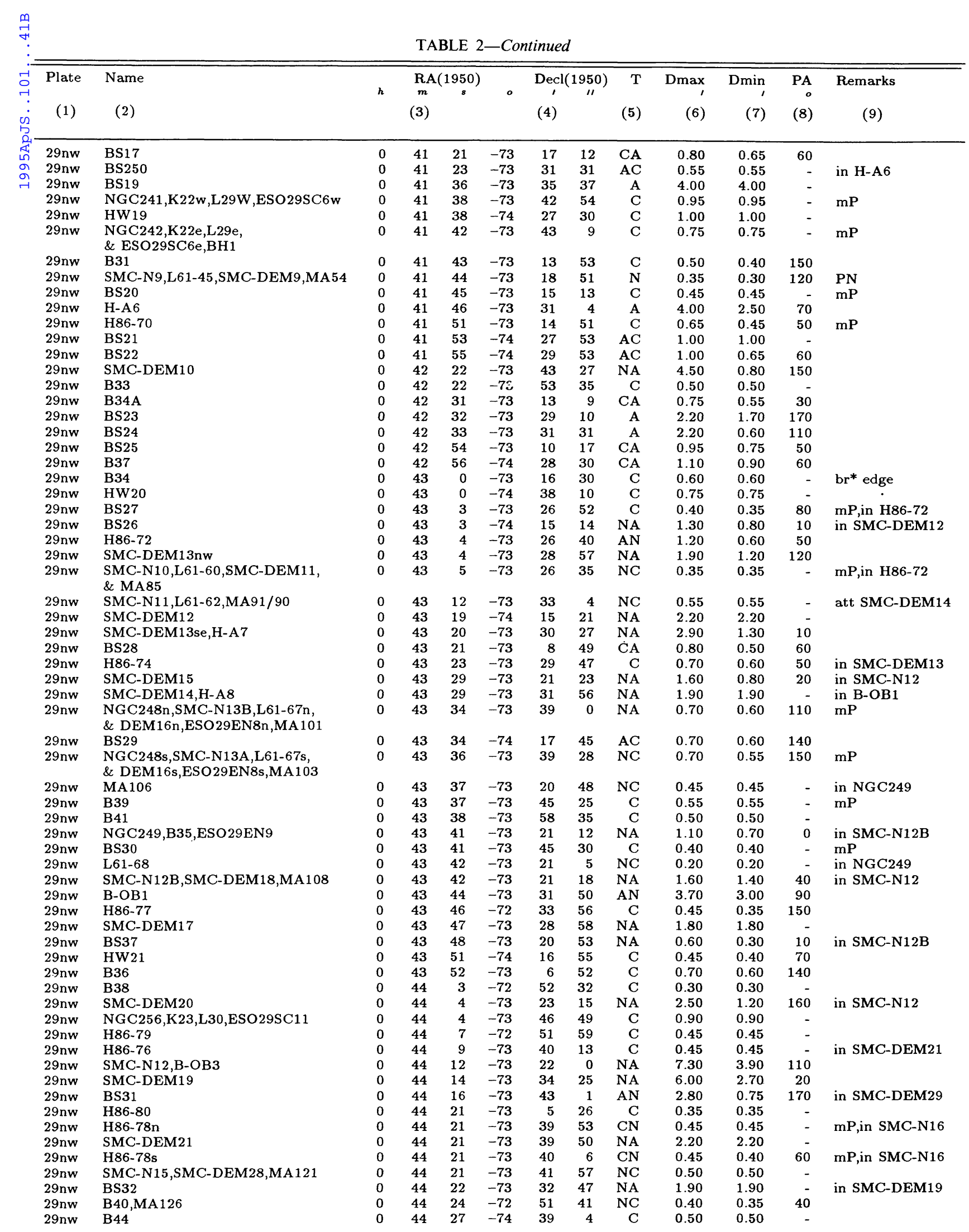


TABLE 2-Continued

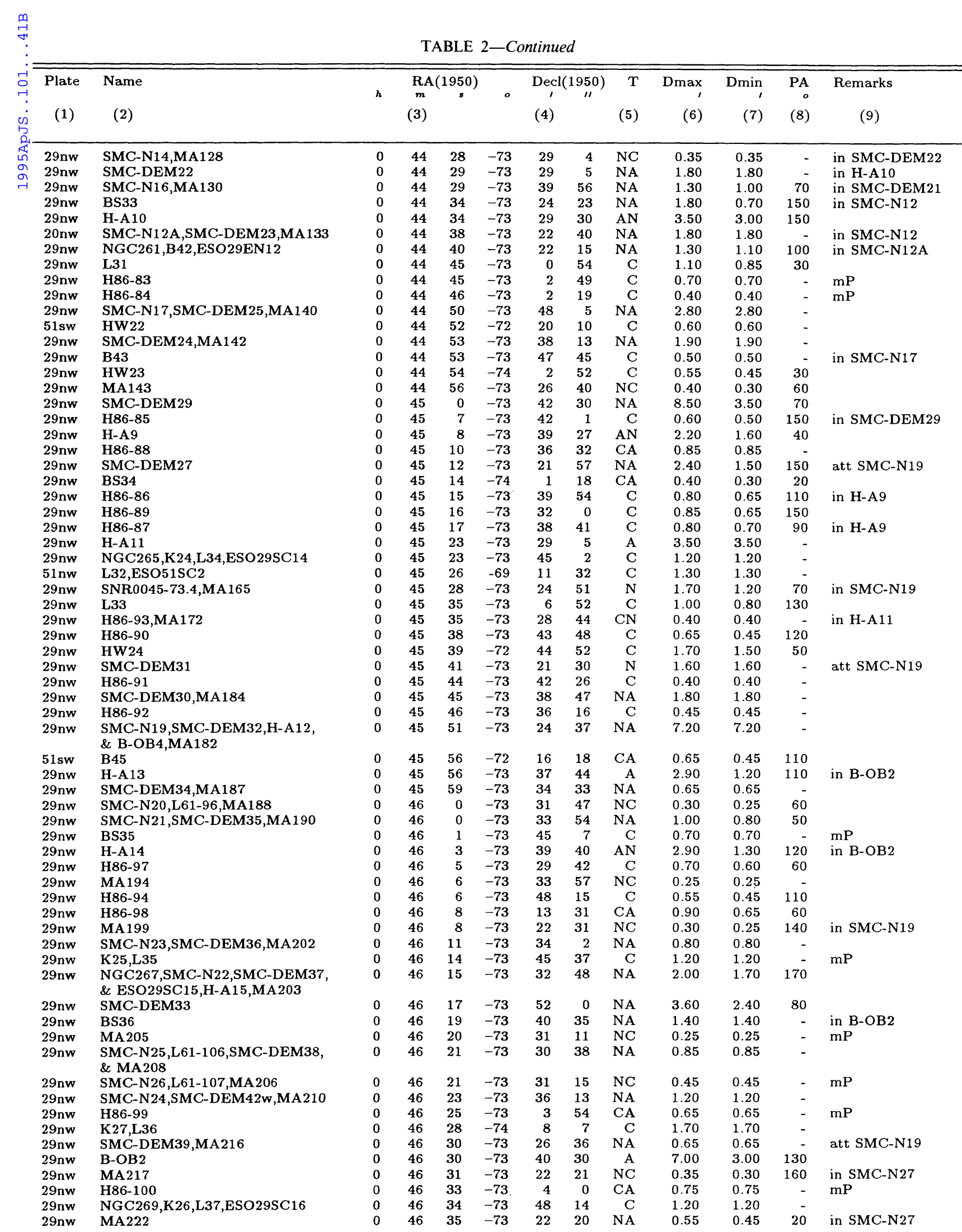


TABLE 2-Continued

\begin{tabular}{|c|c|c|c|c|c|c|c|c|c|c|c|c|}
\hline \multirow{2}{*}{$\begin{array}{l}\text { Plate } \\
\text { (1) }\end{array}$} & \multirow{2}{*}{$\begin{array}{c}\text { Name } \\
(2)\end{array}$} & \multicolumn{4}{|c|}{ RA(1950) } & \multicolumn{2}{|c|}{$\operatorname{Decl}(1950)$} & \multirow{2}{*}{$\begin{array}{r}\mathrm{T} \\
(5)\end{array}$} & \multirow{2}{*}{$\begin{array}{r}\text { Dmax } \\
(6)\end{array}$} & \multirow{2}{*}{$\begin{array}{r}\text { Dmin } \\
(7)\end{array}$} & \multirow{2}{*}{$\begin{array}{r}\text { PA } \\
0 \\
(8)\end{array}$} & \multirow{2}{*}{$\begin{array}{c}\text { Remarks } \\
\text { (9) }\end{array}$} \\
\hline & & & (3) & & & (4) & & & & & & \\
\hline $29 n w$ & BS38 & 0 & 46 & 35 & -73 & 35 & 56 & $\mathrm{CN}$ & 0.40 & 0.20 & 150 & att SMC-N24 \\
\hline $29 n w$ & SMC-N27,SMC-DEM40,MA225 & 0 & 46 & 36 & -73 & 22 & 17 & NA & 1.00 & 1.00 & - & \\
\hline $29 n w$ & SMC-DEM46w & 0 & 46 & 37 & -73 & 1 & 30 & NA & 4.00 & 2.40 & 120 & \\
\hline $29 \mathrm{nw}$ & $\begin{array}{l}\text { SMC-DEM42e,ESO29snr17 } \\
\& \text { SNR0046-73.5 }\end{array}$ & 0 & 46 & 38 & -73 & 35 & 37 & $\mathrm{~N}$ & 2.00 & 1.40 & 120 & att SMC-N24 \\
\hline $29 n w$ & MA228 & 0 & 46 & 39 & -73 & 22 & 22 & $\mathrm{NC}$ & 0.40 & 0.35 & 80 & in SMC-N27 \\
\hline $29 \mathrm{nw}$ & MA231 & 0 & 46 & 42 & -73 & 22 & 50 & $\mathrm{NC}$ & 0.45 & 0.45 & - & \\
\hline $29 \mathrm{nw}$ & $\mathrm{H} 86-101$ & 0 & 46 & 44 & -73 & 2 & 3 & $\mathrm{C}$ & 0.50 & 0.50 & - & in SMC-DEM46w \\
\hline $29 \mathrm{nw}$ & SMC-N28A,MA234 & 0 & 46 & 44 & -73 & 32 & 26 & NA & 0.90 & 0.75 & 90 & in SMC-N28 \\
\hline $29 n w$ & $\mathrm{~B} 47$ & 0 & 46 & 46 & -73 & 34 & 51 & $\mathrm{C}$ & 1.00 & 1.00 & - & \\
\hline $29 \mathrm{nw}$ & SMC-N28,SMC-DEM43,MA244 & 0 & 46 & 47 & -73 & 31 & 52 & NA & 1.80 & 1.80 & - & \\
\hline $29 \mathrm{nw}$ & B48 & 0 & 46 & 50 & -73 & 41 & 26 & $\mathrm{CA}$ & 1.30 & 1.10 & 140 & in $\mathrm{H}-\mathrm{A} 16$ \\
\hline $29 \mathrm{nw}$ & MA246 & 0 & 46 & 52 & -73 & 32 & 11 & NA & 0.50 & 0.40 & 10 & in $\mathrm{SMC}-\mathrm{N} 28$ \\
\hline $29 \mathrm{nw}$ & H-A16 & 0 & 46 & 54 & -73 & 42 & 10 & A & 2.40 & 1.80 & 150 & in $\mathrm{B}-\mathrm{OB} 2$ \\
\hline $51 \mathrm{sw}$ & L38,ESO51SC3 & 0 & 46 & 55 & -70 & 8 & 32 & $\mathrm{C}$ & 1.80 & 1.80 & - & \\
\hline $29 \mathrm{nw}$ & HW 25 & 0 & 47 & 0 & -74 & 34 & 32 & $\mathrm{C}$ & 0.50 & 0.40 & 60 & \\
\hline $29 n w$ & B49 & 0 & 47 & 6 & -73 & 50 & 2 & $\mathrm{C}$ & 0.50 & 0.50 & - & \\
\hline $29 \mathrm{nw}$ & SMC-N30A,L61-126,MA267 & 0 & 47 & 10 & -73 & 26 & 13 & $\mathrm{NC}$ & 0.40 & 0.40 & - & att SMC-N30 \\
\hline $29 \mathrm{nw}$ & SMC-N30,SMC-DEM45,MA282/265 & 0 & 47 & 13 & -73 & 24 & 41 & NA & 2.70 & 2.50 & 135 & \\
\hline $29 n w$ & SNR0047-73.5 & 0 & 47 & 16 & -73 & 30 & 58 & $\mathrm{~N}$ & 2.90 & 2.30 & 45 & att SMC-DEM49 \\
\hline $29 n w$ & B50 & 0 & 47 & 16 & -73 & 38 & 5 & $\mathrm{C}$ & 0.55 & 0.55 & - & $\mathrm{mP}$ \\
\hline $29 n w$ & H86-103 & 0 & 47 & 17 & -73 & 19 & 15 & C & 0.55 & 0.45 & 40 & \\
\hline $29 n w$ & BS41 & 0 & 47 & 17 & -73 & 37 & 36 & $\mathrm{C}$ & 0.55 & 0.55 & - & $\mathrm{mP}$ \\
\hline $29 n w$ & $\mathrm{H} 86-104$ & 0 & 47 & 26 & -73 & 22 & 57 & $\mathrm{C}$ & 0.40 & 0.40 & - & \\
\hline $29 n w$ & SMC-DEM47,B-OB6w & 0 & 47 & 28 & -73 & 9 & 9 & NA & 4.20 & 4.20 & - & \\
\hline $29 n w$ & BS42 & 0 & 47 & 29 & -73 & 31 & 7 & $\mathrm{CA}$ & 1.00 & 1.00 & - & in SMC-DE` 149 \\
\hline $29 \mathrm{nw}$ & BS43 & 0 & 47 & 29 & -73 & 38 & 36 & A & 1.10 & 0.80 & 150 & \\
\hline $29 n w$ & L39 & 0 & 47 & 31 & -73 & 38 & 46 & C & 0.70 & 0.55 & 170 & in $\mathrm{BS} 43$ \\
\hline $29 \mathrm{nw}$ & H86-102 & 0 & 47 & 38 & -73 & 48 & 9 & C & 0.55 & 0.55 & - & \\
\hline $29 n w$ & SMC-DEM46e & 0 & 47 & 40 & -73 & 5 & 1 & NA & 3.90 & 2.60 & 120 & \\
\hline $29 n w$ & SMC-DEM44 & 0 & 47 & 41 & -73 & 42 & 10 & NA & 6.70 & 3.90 & 40 & \\
\hline $29 n w$ & SMC-N33,L61-138,MA297 & 0 & 47 & 43 & -73 & 42 & 53 & $\mathrm{NC}$ & 0.30 & 0.25 & 80 & $\mathrm{mP}$,in SMC-DEM44 \\
\hline $29 \mathrm{nw}$ & MA301 & 0 & 47 & 44 & -73 & 42 & 43 & NC & 0.35 & 0.30 & 80 & $\mathrm{mP}$,in SMC-DEM44 \\
\hline $29 n w$ & SMC-DEM49 & 0 & 47 & 50 & -73 & 32 & 10 & NA & 4.20 & 4.20 & - & \\
\hline $29 \mathrm{nw}$ & SMC-DEM48 & 0 & 47 & 53 & -73 & 5 & 8 & NA & 0.70 & 0.55 & 70 & in SMC-DEM46e \\
\hline $29 n w$ & B52 & 0 & 47 & 53 & -73 & 19 & 50 & C & 1.10 & 1.10 & - & in SMC-DEM51 \\
\hline $29 n w$ & HW26 & 0 & 47 & 53 & -73 & 58 & 50 & $\mathrm{CA}$ & 1.20 & 1.00 & 130 & \\
\hline $29 \mathrm{nw}$ & $\begin{array}{l}\text { SMC-N32,L61-137,H86-111, } \\
\text { \& MA316 }\end{array}$ & 0 & 47 & 54 & -73 & 5 & 3 & NC & 0.45 & 0.45 & - & in SMC-DEM48 \\
\hline $29 n w$ & MA317 & 0 & 47 & 56 & -73 & 26 & 57 & $\mathrm{NC}$ & 0.30 & 0.25 & 20 & $\mathrm{mP}$ \\
\hline $29 \mathrm{nw}$ & H86-110 & 0 & 47 & 57 & -73 & 7 & 20 & $\mathrm{CA}$ & 0.75 & 0.55 & 160 & $\mathrm{mP}$ \\
\hline $29 \mathrm{nw}$ & H86-109 & 0 & 47 & 59 & -73 & 8 & 0 & C & 0.45 & 0.45 & - & $\mathrm{mP}$ \\
\hline $29 \mathrm{nw}$ & $\begin{array}{l}\text { SMC-N34,L61-142,SMC-DEM50, } \\
\text { \& MA322 }\end{array}$ & 0 & 48 & 0 & -73 & 26 & 45 & $\mathrm{NC}$ & 0.65 & 0.45 & 120 & $\mathrm{mP}$ \\
\hline $29 n w$ & BS 44 & 0 & 48 & 2 & -73 & 22 & 12 & A & 2.90 & 1.70 & 60 & \\
\hline $29 \mathrm{nw}$ & MA323 & 0 & 48 & 3 & -73 & 4 & 48 & NC & 0.35 & 0.35 & - & in DEM46e \\
\hline $29 n w$ & SMC-DEM58 & 0 & 48 & 13 & -72 & 42 & 30 & NA & 6.00 & 5.00 & 70 & \\
\hline $29 n w$ & H86-107 & 0 & 48 & 15 & -73 & 31 & 38 & $\mathrm{CA}$ & 1.20 & 0.75 & 130 & in SMC-DEM49 \\
\hline $29 n w$ & SMC-DEM56 & 0 & 48 & 16 & -72 & 51 & 40 & NA & 5.60 & 2.10 & 80 & \\
\hline $29 n w$ & SMC-DEM51 & 0 & 48 & 18 & -73 & 19 & 30 & NA & 4.50 & 2.80 & 70 & \\
\hline $29 n w$ & H-A 17 & 0 & 48 & 19 & -73 & 36 & 32 & A & 3.00 & 3.00 & - & \\
\hline $29 n w$ & B53 & 0 & 48 & 19 & -73 & 39 & 7 & C & 0.95 & 0.95 & - & \\
\hline $29 \mathrm{nw}$ & H-A18 & 0 & 48 & 24 & -73 & 26 & 43 & A & 2.80 & 2.10 & 160 & \\
\hline $29 n w$ & H86-112 & 0 & 48 & 26 & -73 & 27 & 47 & C & 0.65 & 0.55 & 0 & in $\mathrm{H}-\mathrm{A} 18$ \\
\hline $29 \mathrm{nw}$ & SMC-N35,SMC-DEM57,MA347 & 0 & 48 & 27 & -72 & 48 & 45 & NA & 1.10 & 0.45 & 170 & \\
\hline $29 \mathrm{nw}$ & BS39 & 0 & 48 & 27 & -73 & 37 & 10 & C & 0.45 & 0.40 & 40 & in $\mathrm{H}-\mathrm{A} 17$ \\
\hline $29 \mathrm{nw}$ & BS45 & 0 & 48 & 30 & -73 & 18 & 20 & $\mathrm{CA}$ & 1.00 & 0.90 & 70 & in SMC-DEM51 \\
\hline $29 \mathrm{nw}$ & MA351 & 0 & 48 & 33 & -73 & 35 & 49 & $\mathrm{NC}$ & 0.45 & 0.40 & 60 & in $\mathrm{H}-\mathrm{A} 17$ \\
\hline $29 \mathrm{nw}$ & $\begin{array}{l}\text { SMC-N36w,SMC-DEM54w,H-A19, } \\
\& \text { B-OB6ct,MA358 }\end{array}$ & 0 & 48 & 35 & -73 & 9 & 11 & NA & 3.90 & 3.40 & 170 & \\
\hline $29 n w$ & $\mathrm{H}-\mathrm{A} 20$ & 0 & 48 & 37 & -72 & 54 & 10 & A & 3.00 & 3.00 & - & \\
\hline $29 n w$ & B55 & 0 & 48 & 39 & -73 & 39 & 23 & $\mathrm{C}$ & 0.70 & 0.60 & 110 & \\
\hline $29 n w$ & B54 & 0 & 48 & 44 & -73 & 28 & 42 & $\mathrm{C}$ & 0.65 & 0.65 & - & \\
\hline $29 n w$ & H86-114 & 0 & 48 & 45 & -72 & 55 & 28 & C & 0.75 & 0.65 & 150 & in $\mathrm{H}-\mathbf{A} 20$ \\
\hline $29 n w$ & H86-106w & 0 & 48 & 46 & -73 & 36 & 30 & $\mathrm{C}$ & 0.50 & 0.40 & 90 & mP,in SMC-DEM52 \\
\hline $29 n w$ & SMC-DEM52,MA370 & 0 & 48 & 49 & -73 & 36 & 38 & NA & 1.40 & 1.20 & 50 & \\
\hline $29 n w$ & H86-115 & 0 & 48 & 50 & -73 & 19 & 27 & $\mathrm{AC}$ & 1.60 & 1.20 & 40 & in SMC-DEM51 \\
\hline $29 n w$ & SMC-N37,SMC-DEM55,H-A23 & 0 & 48 & 52 & -73 & 4 & 3 & NA & 6.10 & 3.90 & 90 & \\
\hline $29 \mathrm{nw}$ & $\mathrm{H} 86-106 \mathrm{e}$ & 0 & 48 & 52 & -73 & 36 & 30 & $\mathrm{C}$ & 0.55 & 0.45 & 80 & $\mathrm{mP}$,in SMC-DEM52 \\
\hline $29 n w$ & HW27 & 0 & 48 & 52 & -74 & 53 & 10 & $\mathrm{C}$ & 0.65 & 0.65 & - & \\
\hline
\end{tabular}


TABLE 2-Continued

\begin{tabular}{|c|c|c|c|c|c|c|c|c|c|c|c|c|}
\hline \multirow{3}{*}{ 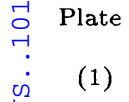 } & \multirow{3}{*}{$\begin{array}{c}\text { Name } \\
\text { (2) }\end{array}$} & \multicolumn{4}{|c|}{$\mathrm{RA}(1950)$} & \multicolumn{2}{|c|}{$\operatorname{Decl}(1950)$} & \multirow{3}{*}{$\begin{array}{r}\mathrm{T} \\
(5)\end{array}$} & \multirow{3}{*}{$\begin{array}{r}\text { Dmax } \\
(6)\end{array}$} & \multirow{3}{*}{$\begin{array}{r}\text { Dmin } \\
(7)\end{array}$} & & Remarks \\
\hline & & $h$ & $m$ & $s$ & $o$ & ' & 11 & & & & o & \\
\hline & & & (3) & & & (4) & & & & & (8) & (9) \\
\hline $29 \mathrm{nw}$ & BS46 & 0 & 48 & 53 & -73 & 14 & 31 & $\mathrm{C}$ & 0.50 & 0.45 & 60 & in $\mathbf{L H} 40$ \\
\hline $29 \mathrm{nw}$ & H86-116 & 0 & 48 & 55 & -73 & 13 & 45 & $\mathrm{C}$ & 0.50 & 0.50 & - & in $\mathrm{LH} 40$ \\
\hline $29 \mathrm{nw}$ & L40,H-A22 & 0 & 48 & 56 & -73 & 14 & 13 & $\mathrm{~A}$ & 1.80 & 1.70 & 10 & \\
\hline $29 n w$ & MA384 & 0 & 48 & 57 & -73 & 3 & 14 & $\mathrm{NC}$ & 0.40 & 0.35 & 140 & in $\mathrm{SMC}-\mathrm{N} 37$ \\
\hline $29 n w$ & H-A21 & 0 & 48 & 57 & -73 & 44 & 5 & A & 3.50 & 2.40 & 90 & \\
\hline $29 n w$ & HW 28 & 0 & 48 & 58 & -74 & 23 & 21 & $\mathrm{CA}$ & 0.55 & 0.45 & 150 & in BS50 \\
\hline $29 n w$ & H-A24 & 0 & 49 & 1 & -73 & 34 & 9 & A & 2.50 & 2.50 & - & \\
\hline $29 n w$ & BS48 & 0 & 49 & 1 & -73 & 40 & 18 & $\mathrm{AC}$ & 0.85 & 0.55 & 80 & mP,in SMC-DEM53 \\
\hline $29 n w$ & BS47 & 0 & 49 & 5 & -72 & 26 & 26 & NA & 2.10 & 1.70 & 100 & in SMC-DEM66 \\
\hline $29 n w$ & SMC-N36e,SMC-DEM54e,B-OB6e & 0 & 49 & 6 & -73 & 8 & 41 & $\mathrm{NA}$ & 4.20 & 2.90 & 150 & \\
\hline $29 n w$ & L41 & 0 & 49 & 9 & -72 & 59 & 56 & $\mathrm{C}$ & 0.65 & 0.65 & - & in $\mathrm{H}-\mathrm{A} 25$ \\
\hline $29 n w$ & H86-108,MA401 & 0 & 49 & 9 & -73 & 40 & 41 & NA & 1.00 & 1.00 & - & $\mathrm{mP}$,in SMC-DEM53 \\
\hline $29 n w$ & BS49 & 0 & 49 & 10 & -73 & 1 & 30 & NA & 1.60 & 1.60 & - & att SMC-N37 \\
\hline $29 n w$ & B56 & 0 & 49 & 12 & -73 & 28 & 42 & $\mathrm{C}$ & 0.45 & 0.45 & - & \\
\hline $29 n w$ & BS40 & 0 & 49 & 13 & -73 & 33 & 19 & CA & 0.90 & 0.80 & 140 & in $\mathrm{H}-\mathrm{A} 24$ \\
\hline $29 n w$ & SMC-N41,L61-166,MA424 & 0 & 49 & 14 & -73 & 9 & 23 & $\mathrm{NC}$ & 0.35 & 0.35 & - & in $\mathrm{SMC}-\mathrm{N36e}$ \\
\hline $29 n w$ & $\mathrm{H} 86-105$ & 0 & 49 & 15 & -73 & 46 & 28 & $\mathrm{C}$ & 0.45 & 0.45 & - & \\
\hline $29 n w$ & SMC-DEM53 & 0 & 49 & 21 & -73 & 39 & 51 & NA & 3.50 & 2.50 & 40 & \\
\hline $29 n w$ & BS50 & 0 & 49 & 23 & -74 & 23 & 21 & A & 4.40 & 3.60 & 80 & \\
\hline $29 n w$ & SNR0049-73.6 & 0 & 49 & 27 & -73 & 38 & 24 & $\mathrm{~N}$ & 1.90 & 1.10 & 70 & \\
\hline $29 n w$ & H-A25 & 0 & 49 & 28 & -72 & 57 & 53 & NA & 6.00 & 4.50 & 40 & in SMC-DEM63 \\
\hline $29 n w$ & NGC290,L42,ESO29SC19 & 0 & 49 & 30 & -73 & 26 & 2 & $\mathrm{C}$ & 1.10 & 1.10 & - & in BS51 \\
\hline $29 n w$ & H86-113 & 0 & 49 & 32 & -73 & 51 & 44 & $\mathrm{C}$ & 0.40 & 0.40 & - & \\
\hline $29 n w$ & H86-121 & 0 & 49 & 36 & -73 & 24 & 40 & $\mathrm{C}$ & 0.55 & 0.55 & - & in BS51 \\
\hline $29 n w$ & SMC-DEM59 & 0 & 49 & 36 & -73 & 46 & 42 & NA & 1.40 & 1.35 & 90 & \\
\hline $29 n w$ & BS251 & 0 & 49 & 41 & -73 & 33 & 28 & $\mathrm{CA}$ & 0.40 & 0.35 & 140 & \\
\hline $29 n w$ & $\mathrm{H}-\mathrm{A} 27 \mathrm{~A}$ & 0 & 49 & 43 & -73 & 21 & 30 & A & 3.10 & 2.20 & 50 & \\
\hline $20 \mathrm{nw}$ & BS51 & 0 & 49 & 43 & -73 & 25 & 42 & A & 3.20 & 3.20 & - & \\
\hline $29 n w$ & BS252 & 0 & 49 & 46 & -73 & 36 & 26 & $\mathrm{AC}$ & 0.65 & 0.45 & 80 & \\
\hline $29 n w$ & BS52 & 0 & 49 & 46 & -73 & 57 & 47 & $\mathrm{CA}$ & 1.20 & 0.90 & 120 & \\
\hline $29 n w$ & B-OB7 & 0 & 49 & 47 & -73 & 3 & 57 & AN & 4.00 & 2.00 & 40 & att SMC-N37 \\
\hline $29 \mathrm{nw}$ & H86-124 & 0 & 49 & 49 & -73 & 15 & 5 & $\mathrm{C}$ & 0.85 & 0.65 & 80 & \\
\hline $29 n w$ & H86-119 & 0 & 49 & 50 & -72 & 48 & 21 & $\mathrm{C}$ & 0.65 & 0.55 & 20 & in $\mathrm{B}-\mathrm{OB} 9$ \\
\hline $29 n w$ & B57 & 0 & 49 & 52 & -73 & 17 & 17 & $\mathrm{C}$ & 1.20 & 1.20 & - & \\
\hline $51 \mathrm{sw}$ & K28,L43,ESO51SC4 & 0 & 49 & 54 & -72 & 16 & 10 & $\mathrm{C}$ & 1.70 & 1.70 & - & \\
\hline $29 n w$ & $\begin{array}{l}\text { SMC-N45,L61-189,B60, } \\
\text { \& SMC-DEM60,MA485 }\end{array}$ & 0 & 49 & 56 & -73 & 29 & 53 & $\mathrm{NC}$ & 0.75 & 0.75 & - & in $\mathrm{H}-\mathrm{A} 26$ \\
\hline $29 n w$ & B59,L61-183,MA488 & 0 & 49 & 57 & -73 & 6 & 44 & $\mathrm{NC}$ & 0.80 & 0.60 & 80 & $\mathrm{mP}$ \\
\hline $29 n w$ & H-A26 & 0 & 49 & 58 & -73 & 30 & 9 & $\mathrm{~A}$ & 2.00 & 1.20 & 140 & \\
\hline $29 \mathrm{nw}$ & H86-117 & 0 & 49 & 58 & -73 & 48 & 10 & $\mathrm{C}$ & 0.45 & 0.45 & - & \\
\hline $51 \mathrm{sw}$ & H-A27 & 0 & 50 & 1 & -72 & 24 & 0 & AN & 5.50 & 4.50 & 120 & in SMC-DEM66 \\
\hline $29 \mathrm{nw}$ & $\mathrm{H} 86-123$ & 0 & 50 & 1 & -73 & 26 & 29 & $\mathrm{C}$ & 0.55 & 0.55 & - & in BS51 \\
\hline 29 nw & $\begin{array}{l}\text { SMC-N46,L61-184,SMC-DEM62, } \\
\text { \& MA498 }\end{array}$ & 0 & 50 & 2 & -73 & 7 & 5 & $\mathrm{NC}$ & 0.65 & 0.65 & - & $\mathrm{mP}$ \\
\hline 29 nw & $\mathrm{H} 86-120$ & 0 & 50 & 3 & -73 & 44 & 19 & $\mathrm{C}$ & 0.35 & 0.35 & - & in SMC-DEM70s \\
\hline $29 n w$ & H86-127 & 0 & 50 & 4 & -72 & 48 & 25 & $\mathrm{C}$ & 0.60 & 0.40 & 20 & in $\mathrm{B}-\mathrm{OB} 9$ \\
\hline $29 n w$ & B-OB9 & 0 & 50 & 5 & -72 & 49 & 10 & A & 2.80 & 2.50 & 80 & in SMC-DEM64 \\
\hline $29 n w$ & SMC-DEM63,B-OB8 & 0 & 50 & 6 & -72 & 57 & 1 & NA & 11.0 & 5.00 & 100 & \\
\hline $29 n w$ & BS53 & 0 & 50 & 6 & -73 & 44 & 56 & $\mathrm{~A}$ & 0.65 & 0.55 & 150 & in SMC-DEM70s \\
\hline $29 n w$ & $\mathrm{~K} 29, \mathrm{~L} 44$ & 0 & 50 & 8 & -73 & 13 & 32 & $\mathrm{C}$ & 0.95 & 0.95 & - & \\
\hline $29 \mathrm{nw}$ & H86-126 & 0 & 50 & 8 & -73 & 22 & 7 & $\mathrm{C}$ & 0.50 & 0.50 & - & \\
\hline $29 \mathrm{nw}$ & SMC-DEM66,B-OB 10 & 0 & 50 & 12 & -72 & 25 & 0 & NA & 18.0 & 15.0 & 170 & \\
\hline $29 n w$ & SMC-DEM65,MA523 & 0 & 50 & 13 & -72 & 33 & 1 & NA & 2.00 & 1.80 & 100 & att SMC-DEM66 \\
\hline $29 n w$ & SMC-DEM64 & 0 & 50 & 15 & -72 & 44 & 0 & NA & 11.0 & 5.00 & 10 & \\
\hline $29 n w$ & H86-122 & 0 & 50 & 15 & -73 & 43 & 59 & $\mathrm{C}$ & 0.45 & 0.45 & - & in SMC-DEM70s \\
\hline $51 \mathrm{sw}$ & SMC-DEM 67 & 0 & 50 & 16 & -72 & 7 & 46 & NA & 3.00 & 2.50 & 100 & \\
\hline $29 \mathrm{nw}$ & H86-128 & 0 & 50 & 16 & -73 & 5 & 25 & $\mathrm{C}$ & 0.45 & 0.45 & - & \\
\hline $29 n w$ & MA524 & 0 & 50 & 18 & -72 & 55 & 50 & NA & 3.00 & 2.60 & 160 & in SMC-DEM63 \\
\hline $29 \mathrm{nw}$ & H-A34 & 0 & 50 & 22 & -72 & 39 & 19 & $\mathrm{AN}$ & 2.80 & 2.00 & 30 & in SMC-DEM64 \\
\hline $51 \mathrm{sw}$ & $\mathrm{B} 61$ & 0 & 50 & 23 & -72 & 7 & 40 & $\mathrm{AN}$ & 1.80 & 1.20 & 150 & in SMC-DEM67 \\
\hline $29 \mathrm{nw}$ & SMC-DEM70s & 0 & 50 & 23 & -73 & 43 & 0 & NA & 7.00 & 2.50 & 30 & \\
\hline $29 \mathrm{nw}$ & BS54 & 0 & 50 & 25 & -73 & 35 & 13 & $\mathrm{AC}$ & 0.55 & 0.50 & 100 & in $\mathrm{H}-\mathrm{A} 28$ \\
\hline $29 n w$ & B62 & 0 & 50 & 26 & -73 & 52 & 24 & A & 1.20 & 0.80 & 80 & \\
\hline $29 \mathrm{nw}$ & BS55 & 0 & 50 & 27 & -72 & 29 & 59 & A & 2.40 & 1.10 & 20 & in SMC-DEM66 \\
\hline $29 n w$ & H86-129 & 0 & 50 & 27 & -72 & 48 & 3 & $\mathrm{C}$ & 0.65 & 0.45 & 40 & in SMC-DEM64 \\
\hline $29 \mathrm{nw}$ & BS56 & 0 & 50 & 29 & -73 & 16 & 38 & C & 0.70 & 0.55 & 90 & \\
\hline $29 \mathrm{nw}$ & BS253 & 0 & 50 & 30 & -73 & 2 & 14 & $\mathrm{AC}$ & 0.60 & 0.40 & 10 & \\
\hline 29 nw & SMC-DEM70n & 0 & 50 & 30 & -73 & 34 & 13 & NA & 10.0 & 4.00 & 0 & \\
\hline 29 nw & H86-125 & 0 & 50 & 30 & -73 & 38 & 41 & $\mathrm{CA}$ & 0.75 & 0.50 & 80 & in SMC-DEM70n \\
\hline $29 \mathrm{nw}$ & SMC-N49,MA547/548 & 0 & 50 & 30 & -73 & 52 & 21 & $\mathrm{NC}$ & 0.30 & 0.25 & 170 & in $\mathrm{B} 62$ \\
\hline
\end{tabular}


TABLE 2-Continued

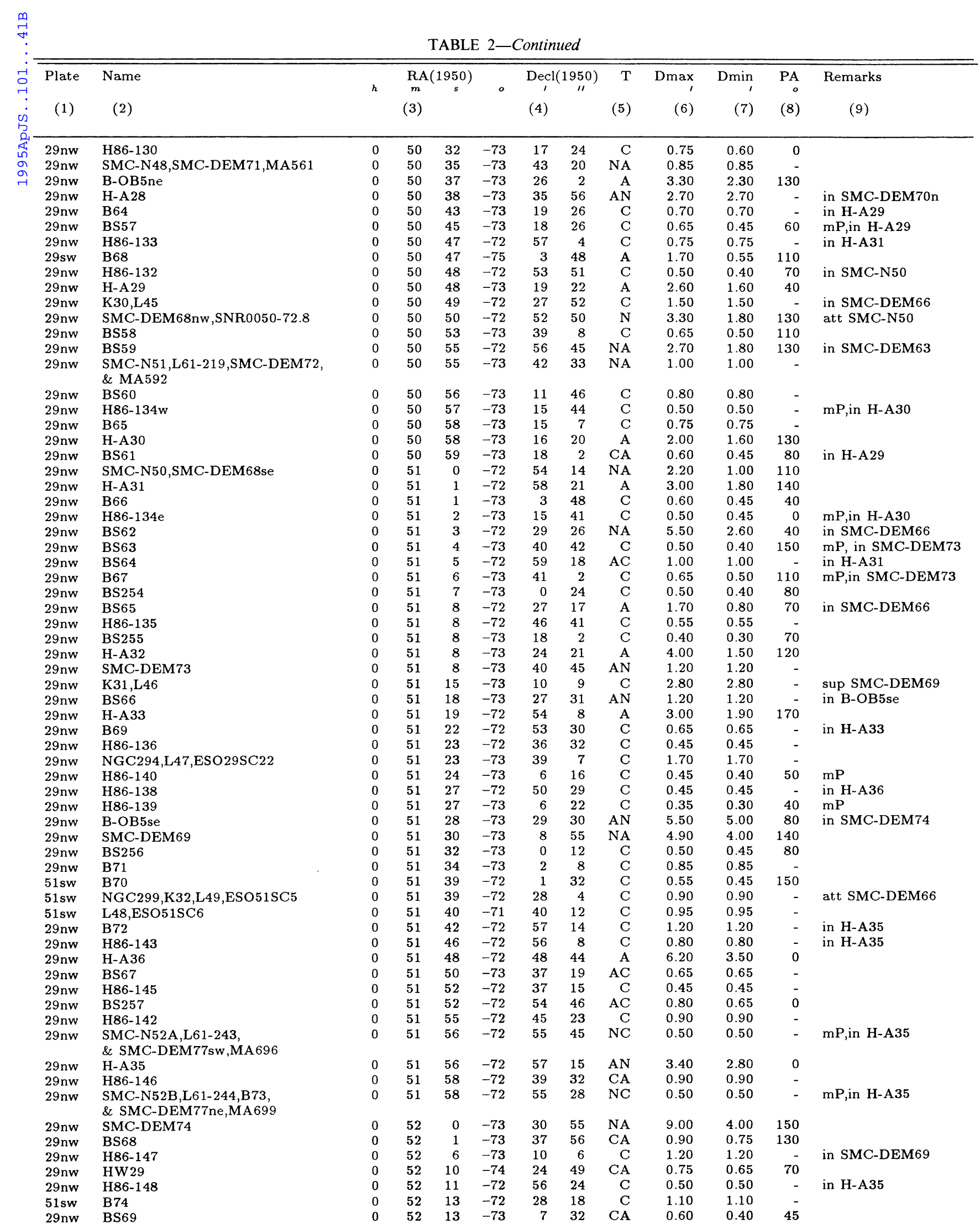


TABLE 2-Continued

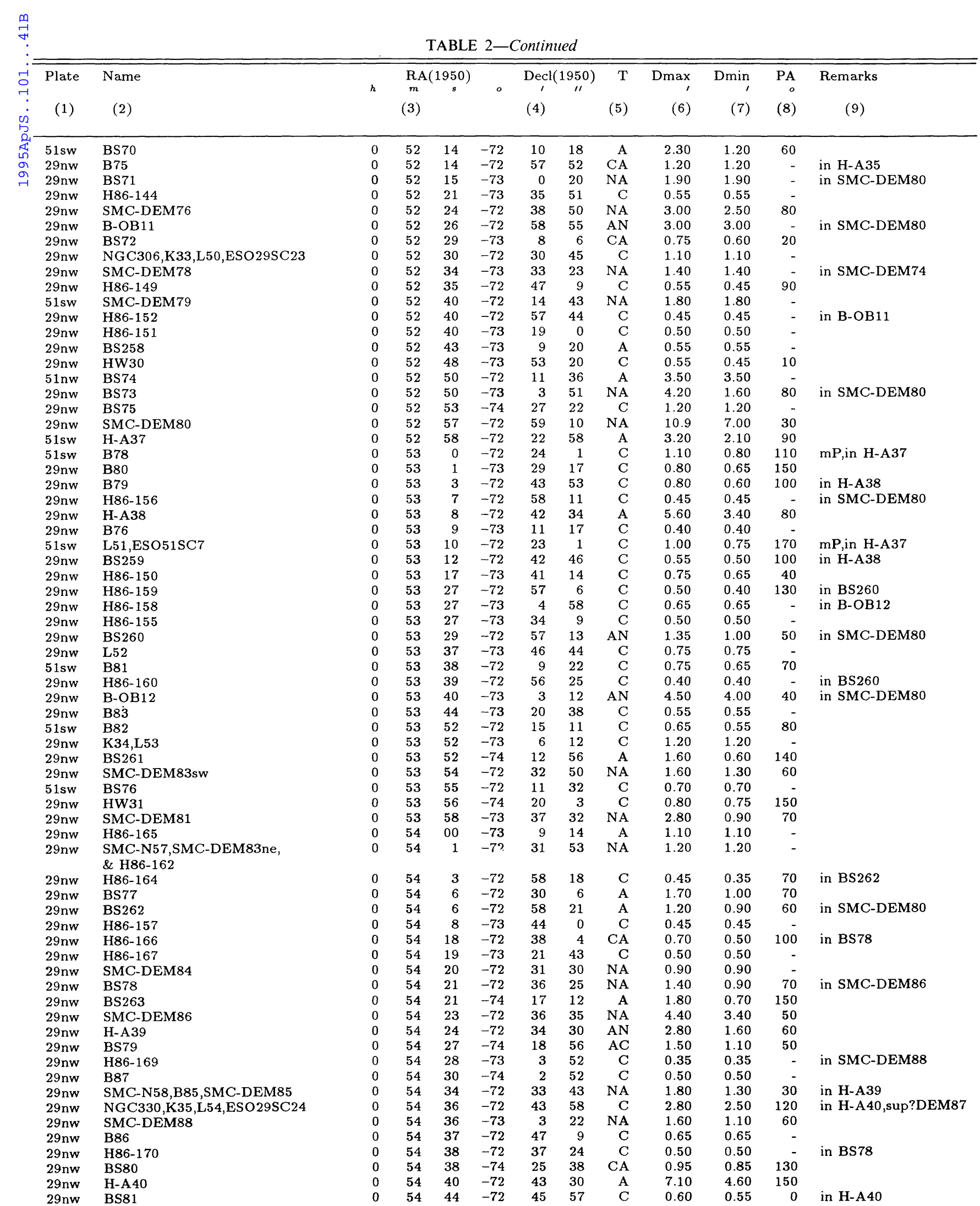


TABLE 2-Continued

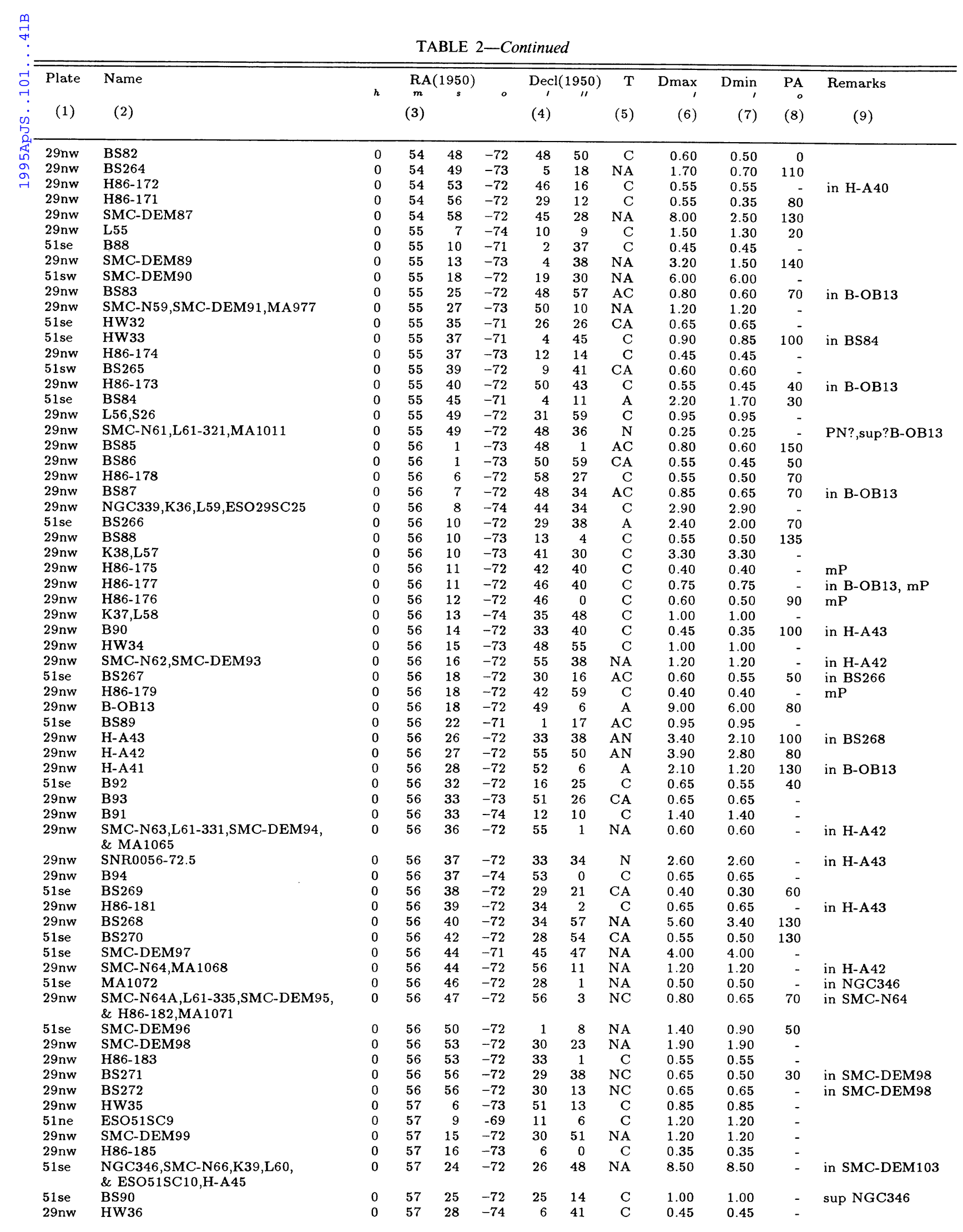


TABLE 2-Continued

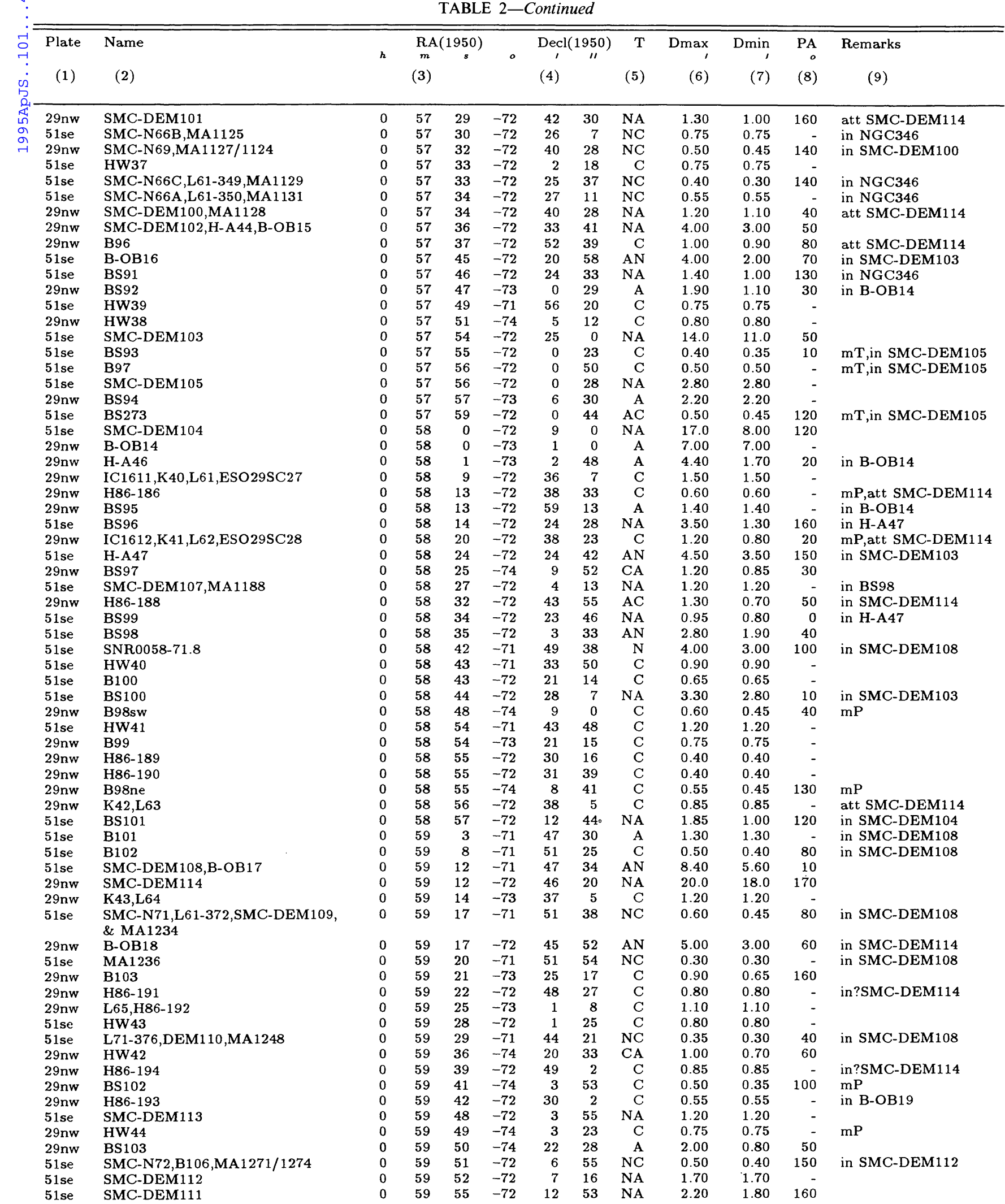


TABLE 2-Continued

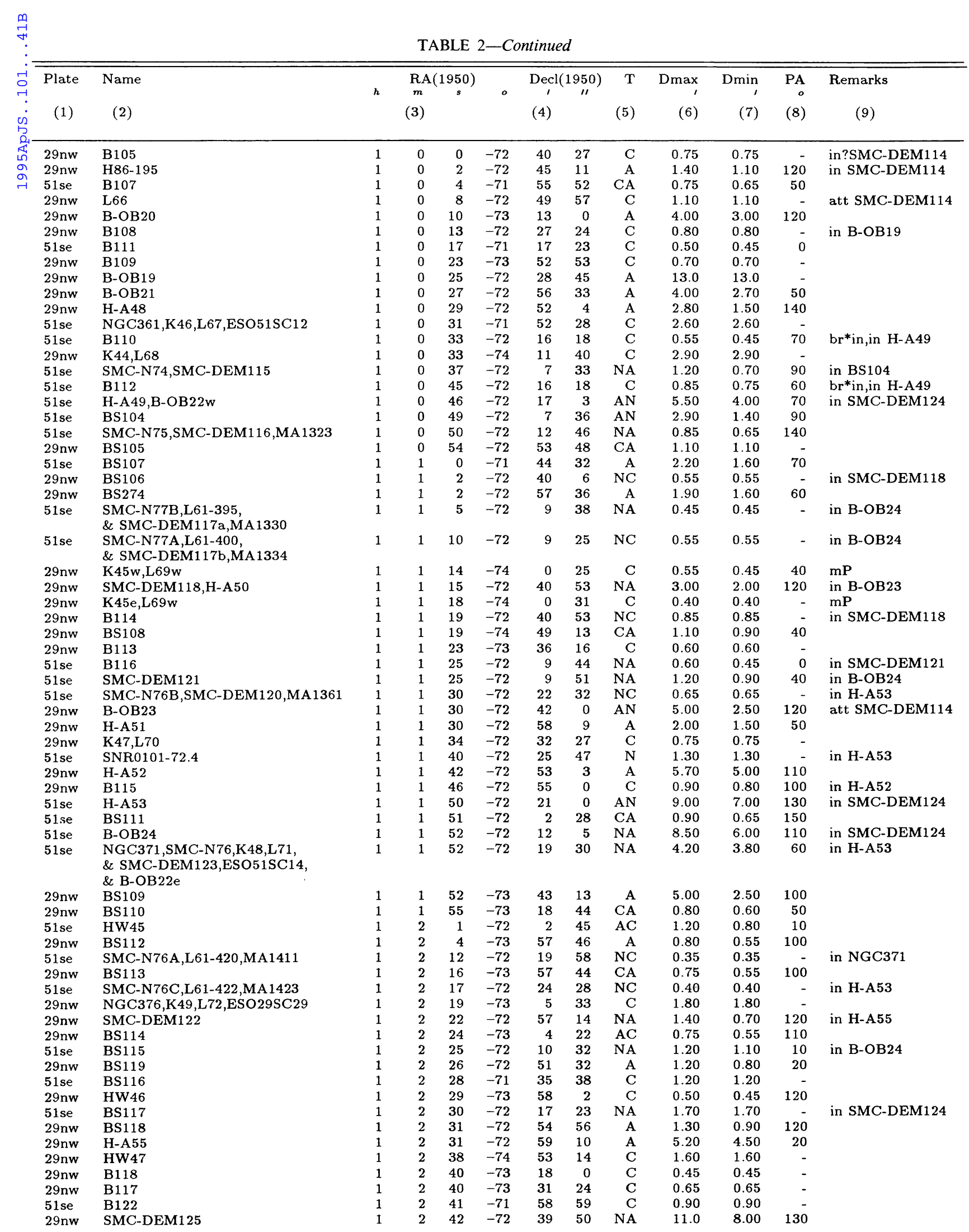


TABLE 2-Continued

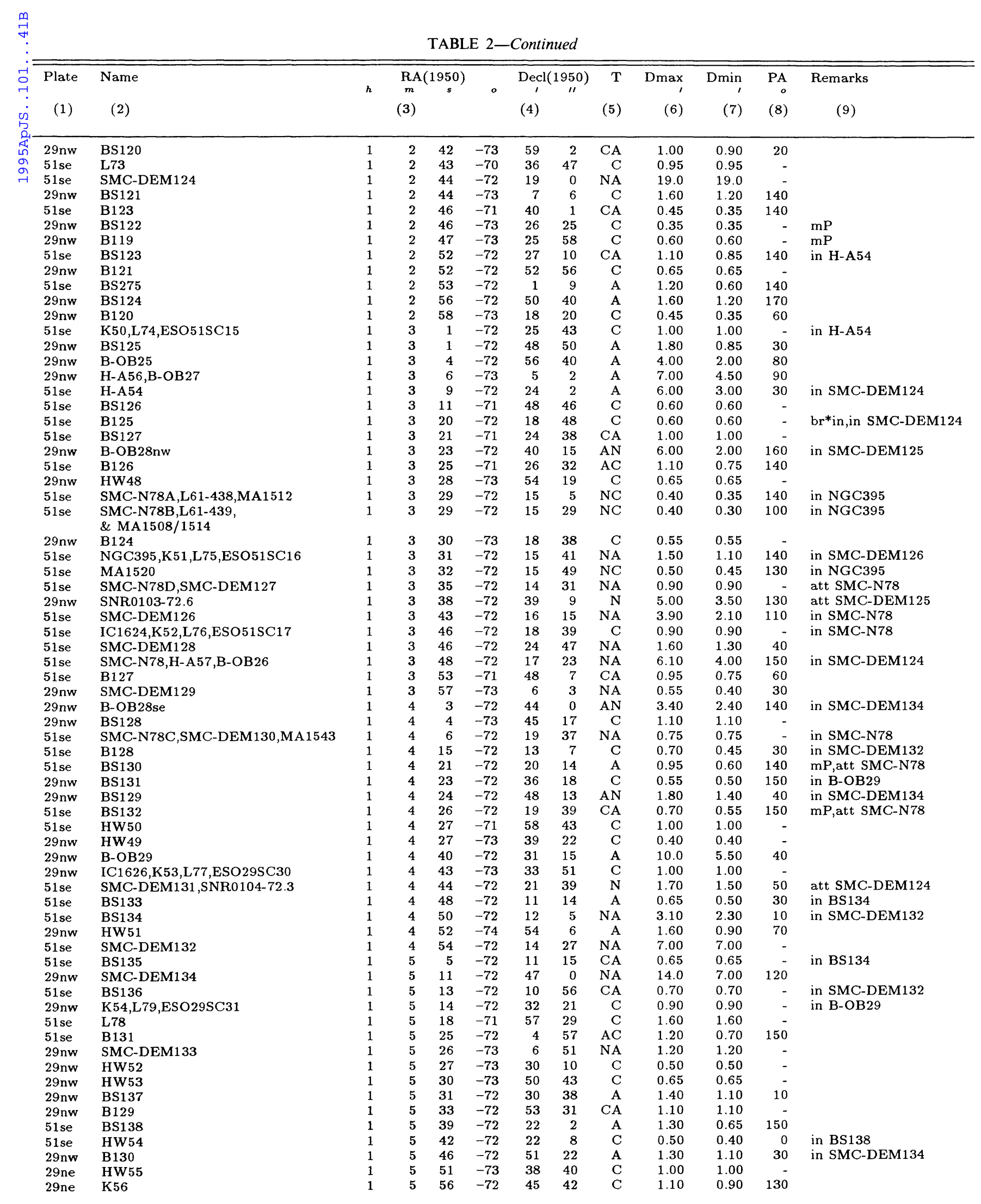


TABLE 2-Continued

\begin{tabular}{|c|c|c|c|c|c|c|c|c|c|c|c|c|c|}
\hline \multirow{3}{*}{ 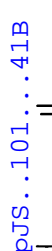 } & \multicolumn{13}{|c|}{ TABLE 2-Continued } \\
\hline & Plate & Name & & RA & 1950 & & Decl & 1950) & $\mathrm{T}$ & Dmax & Dmin & PA & Remarks \\
\hline & (1) & $(2)$ & $\boldsymbol{h}$ & $\begin{array}{r}m \\
(3)\end{array}$ & $s$ & $o$ & $\begin{array}{r}\prime \\
(4)\end{array}$ & $" \prime$ & (5) & (6) & (7) & $\begin{array}{r}o \\
(8)\end{array}$ & $(9)$ \\
\hline 客: & 29 ne & L80 & 1 & 5 & 58 & -73 & 2 & 11 & $\mathrm{C}$ & 1.20 & 1.20 & - & \\
\hline '⿳亠口冋!' & $51 \mathrm{se}$ & HW56 & 1 & 6 & 4 & -71 & 12 & 10 & C & 0.65 & 0.65 & - & \\
\hline $\ln (\rightarrow 1$ & 29 ne & SMC-N79,L61-471,MA1634 & 1 & 6 & 5 & -72 & 50 & 47 & $\mathrm{NC}$ & 0.30 & 0.30 & - & in $\mathbf{H}-\mathbf{A} 58$ \\
\hline-3 & 29 ne & K55,L81 & 1 & 6 & 5 & -73 & 23 & 13 & $\mathrm{C}$ & 0.95 & 0.95 & - & \\
\hline & 29 ne & B-OB30 & 1 & 6 & 7 & -72 & 32 & 10 & $\mathbf{A}$ & 4.00 & 3.00 & 50 & \\
\hline & 29 ne & H-A58 & 1 & 6 & 9 & -72 & 50 & 0 & $\mathbf{A}$ & 5.00 & 3.00 & 40 & in SMC-DEM134 \\
\hline & $51 \mathrm{se}$ & HW57 & 1 & 6 & 10 & -72 & 8 & 51 & $\mathrm{C}$ & 1.40 & 1.40 & - & \\
\hline & $51 \mathrm{se}$ & NGC411,K60,L82,ESO51SC19 & 1 & 6 & 21 & -72 & 2 & 8 & $\mathrm{C}$ & 2.10 & 1.90 & 150 & \\
\hline & 29ne & NGC416,K59,L83,ESO29SC32 & 1 & 6 & 26 & -72 & 37 & 21 & $\mathrm{C}$ & 1.70 & 1.70 & - & \\
\hline & 51 ne & BS139 & 1 & 6 & 36 & -72 & 12 & 57 & $\mathrm{AC}$ & 0.65 & 0.55 & 100 & in $\mathrm{SMC}-\mathrm{N} 80$ \\
\hline & $51 \mathrm{se}$ & $\begin{array}{l}\text { SMC-N80,SMC-DEM135,H-A59, } \\
\& \text { MA1654 }\end{array}$ & 1 & 6 & 36 & -72 & 15 & 32 & NA & 5.10 & 4.00 & 50 & \\
\hline & 29 ne & $\mathrm{B} 132$ & 1 & 6 & 38 & -73 & 47 & 25 & $\mathrm{CA}$ & 0.55 & 0.55 & - & \\
\hline & 29 ne & HW 58 & 1 & 6 & 39 & -73 & 57 & 49 & $\mathrm{AC}$ & 0.70 & 0.70 & - & \\
\hline & 29 ne & $\mathrm{K} 57, \mathrm{~L} 86$ & 1 & 6 & 46 & -73 & 31 & 26 & $\mathrm{C}$ & 1.20 & 1.10 & 50 & \\
\hline & 29 ne & NGC419,K58,L85,ESO29SC33 & 1 & 6 & 47 & -73 & 9 & 0 & $\mathrm{C}$ & 2.80 & 2.80 & - & \\
\hline & $51 \mathrm{se}$ & H86-196 & 1 & 6 & 51 & -72 & 6 & 16 & $\mathrm{C}$ & 0.65 & 0.65 & - & \\
\hline & 51 se & L84,ESO51SC20 & 1 & 6 & 51 & -72 & 15 & 42 & NA & 1.90 & 1.10 & 0 & in $\mathrm{SMC}-\mathrm{N} 80$ \\
\hline & $51 \mathrm{se}$ & SMC-N80A,MA1662 & 1 & 6 & 54 & -72 & 16 & 18 & NA & 0.55 & 0.40 & 120 & in L84 \\
\hline & $51 \mathrm{se}$ & L61-476,MA 1673 & 1 & 7 & 2 & -72 & 15 & 53 & NC & 0.25 & 0.20 & 110 & in $\mathrm{L} 84$ \\
\hline & $51 \mathrm{se}$ & B136 & 1 & 7 & 7 & -72 & 1 & 45 & $\mathrm{C}$ & 0.85 & 0.80 & 120 & \\
\hline & $51 \mathrm{se}$ & MA1675 & 1 & 7 & 7 & -72 & 14 & 46 & $\mathrm{NC}$ & 0.20 & 0.20 & - & in $\mathrm{SMC}-\mathrm{N} 80$ \\
\hline & 29 ne & BS 140 & 1 & 7 & 9 & -73 & 58 & 16 & $\mathrm{CA}$ & 0.80 & 0.70 & 30 & \\
\hline & 29ne & HW59 & 1 & 7 & 26 & -73 & 30 & 29 & $\mathrm{CA}$ & 0.55 & 0.50 & 150 & \\
\hline & 29 ne & B134 & 1 & 7 & 33 & -73 & 28 & 23 & $\mathrm{CA}$ & 0.80 & 0.55 & 80 & in $\mathbf{H}-\mathbf{A} 60$ \\
\hline & 29 ne & SMC-DEM138 & 1 & 7 & 35 & -73 & 27 & 25 & NA & 4.60 & 2.80 & 20 & \\
\hline & 29 ne & $\mathrm{K} 61$ & 1 & 7 & 36 & -73 & 21 & 10 & $\mathrm{C}$ & 0.95 & 0.95 & - & \\
\hline & $51 \mathrm{se}$ & BS141 & 1 & 7 & 39 & -72 & 4 & 52 & A & 1.70 & 1.50 & 0 & \\
\hline & 29 ne & BS 142 & 1 & 7 & 39 & -73 & 27 & 59 & $\mathrm{C}$ & 0.40 & 0.35 & 70 & in $\mathbf{H}-\mathbf{A} 60$ \\
\hline & 29 ne & H-A 60 & 1 & 7 & 40 & -73 & 27 & 53 & AN & 2.60 & 1.40 & 40 & in SMC-DEM138 \\
\hline & $51 \mathrm{se}$ & SMC-DEM136 & 1 & 7 & 42 & -72 & 10 & 0 & NA & 8.00 & 6.00 & 140 & \\
\hline & 29 ne & BS143 & 1 & 7 & 44 & -73 & 0 & 3 & $\mathbf{A}$ & 2.50 & 1.80 & 120 & \\
\hline & 29 ne & $\begin{array}{l}\text { IC1644,SMC-N81,L61-481, } \\
\text { \& ESO29EN35,MA1688/1687 }\end{array}$ & 1 & 7 & 44 & -73 & 27 & 40 & $\mathrm{NC}$ & 0.80 & 0.65 & 40 & in $\mathrm{H}-\mathrm{A} 60$ \\
\hline & $51 \mathrm{se}$ & NGC422,K62,L87,ESO51SC22 & 1 & 7 & 52 & -72 & 1 & 58 & $\mathrm{C}$ & 1.00 & 1.00 & - & $\mathrm{mP}$ \\
\hline & 29 ne & B135 & 1 & 7 & 52 & -73 & 27 & 13 & $\mathrm{C}$ & 0.55 & 0.40 & 60 & in $\mathbf{H}-\mathbf{A} 60$ \\
\hline & 29 ne & SMC-DEM137 & 1 & 7 & 54 & -72 & 36 & 50 & NA & 0.90 & 0.75 & 120 & \\
\hline & 51 se & IC1641,HW62,ESO51SC21 & 1 & 8 & 6 & -72 & 2 & 5 & $\mathrm{C}$ & 0.75 & 0.65 & 40 & $\mathrm{mP}$ \\
\hline & 29 ne & HW61 & 1 & 8 & 12 & -72 & 33 & 41 & $\mathrm{C}$ & 0.65 & 0.65 & - & \\
\hline & 29 ne & BS276 & 1 & 8 & 40 & -73 & 0 & 22 & $\mathrm{C}$ & 0.80 & 0.75 & 10 & in SMC-DEM140 \\
\hline & 29 ne & HW63 & 1 & 8 & 46 & -73 & 28 & 30 & $\mathrm{C}$ & 0.75 & 0.75 & - & \\
\hline & $51 \mathrm{se}$ & B139 & 1 & 8 & 55 & -71 & 49 & 38 & $\mathrm{C}$ & 0.65 & 0.55 & 160 & \\
\hline & 29 ne & $\mathrm{B} 137$ & 1 & 9 & 3 & -73 & 13 & 20 & $\mathrm{C}$ & 0.60 & 0.60 & - & \\
\hline & 51 se & BS144 & 1 & 9 & 6 & -71 & 32 & 59 & A & 4.70 & 3.80 & 20 & in $\mathrm{BS} 153$ \\
\hline & 29 ne & BS145 & 1 & 9 & 7 & -72 & 37 & 4 & NA & 1.30 & 0.80 & 140 & \\
\hline & $51 \mathrm{se}$ & HW64 & 1 & 9 & 12 & -71 & 36 & 14 & C & 0.90 & 0.90 & - & in $\mathrm{BS} 153$ \\
\hline & $51 \mathrm{se}$ & B142 & 1 & 9 & 17 & -71 & 44 & 33 & $\mathrm{C}$ & 0.55 & 0.55 & - & in $\mathrm{BS} 153$ \\
\hline & 29 ne & $\mathrm{K} 63, \mathrm{~L} 88$ & 1 & 9 & 20 & -73 & 3 & 33 & $\mathrm{C}$ & 0.90 & 0.90 & - & in SMC-DEM140 \\
\hline & 29 ne & SMC-DEM140 & 1 & 9 & 23 & -73 & 0 & 52 & NA & 12.0 & 12.0 & - & \\
\hline & 29 ne & BS146 & 1 & 9 & 29 & -72 & 36 & 48 & $\mathrm{C}$ & 0.45 & 0.35 & 130 & in SMC-DEM141 \\
\hline & 29 ne & $\mathrm{BS} 147$ & 1 & 9 & 35 & -72 & 59 & 38 & NA & 3.40 & 3.40 & - & in SMC-DEM140 \\
\hline & 29ne & MA1735 & 1 & 9 & 35 & -73 & 1 & 27 & $\mathrm{NC}$ & 0.25 & 0.20 & 40 & in SMC-DEM 140 \\
\hline & 29 ne & BS148 & 1 & 9 & 37 & -72 & 36 & 4 & $\mathrm{CA}$ & 0.50 & 0.40 & 50 & in SMC-DEM141 \\
\hline & 29 ne & SMC-DEM141 & 1 & 9 & 45 & -72 & 37 & 42 & NA & 3.80 & 3.00 & 170 & \\
\hline & 29 ne & B140 & 1 & 9 & 47 & -72 & 38 & 22 & NA & 2.30 & 1.50 & 100 & in SMC-DEM141 \\
\hline & 29 ne & S56,L61-491,MA1740 & 1 & 9 & 51 & -72 & 38 & 51 & $\mathrm{NC}$ & 0.35 & 0.25 & 140 & in $\mathrm{B} 140$ \\
\hline & 29 ne & SMC-DEM142 & 1 & 9 & 55 & -72 & 25 & 43 & NA & 2.10 & 2.10 & - & \\
\hline & $51 \mathrm{se}$ & BS150 & 1 & 10 & 16 & -71 & 40 & 56 & $\mathrm{CA}$ & 0.65 & 0.55 & 10 & in $\mathrm{BS} 153$ \\
\hline & $51 \mathrm{se}$ & IC $1655, L 90, \mathrm{ESO} 51 \mathrm{SC} 23$ & 1 & 10 & 21 & -71 & 35 & 48 & $\mathrm{C}$ & 1.70 & 1.50 & 50 & in $\mathrm{BS} 153$ \\
\hline & $51 \mathrm{se}$ & BS149 & 1 & 10 & 21 & -71 & 37 & 25 & A & 2.80 & 1.20 & 80 & in $\mathrm{BS} 153$ \\
\hline & 51 se & H86-208 & 1 & 10 & 21 & -72 & 11 & 27 & $\mathrm{AC}$ & 0.65 & 0.35 & 140 & \\
\hline & 29 ne & SMC-DEM145 & 1 & 10 & 22 & -73 & 29 & 25 & NA & 2.40 & 2.40 & - & \\
\hline & $51 \mathrm{se}$ & $\mathrm{B} 144, \mathrm{H} 86-200$ & 1 & 10 & 24 & -72 & 12 & 53 & $\mathrm{AC}$ & 0.80 & 0.65 & 70 & $\mathrm{br}^{*}$ in or sup \\
\hline & 29 ne & SMC-DEM144 & 1 & 10 & 24 & -73 & 0 & 14 & NA & 2.30 & 2.30 & - & in SMC-DEM140 \\
\hline & 29 ne & HW65 & 1 & 10 & 29 & -72 & 32 & 10 & C & 0.70 & 0.70 & - & \\
\hline & 29 ne & BS151 & 1 & 10 & 41 & -72 & 58 & 40 & NA & 1.70 & 1.30 & 90 & in SMC-DEM140 \\
\hline & 29ne & SMC-DEM143 & 1 & 10 & 42 & -72 & 31 & 24 & NA & 1.50 & 1.50 & - & \\
\hline & 29 se & HW66,ESO29SC36 & 1 & 10 & 49 & -75 & 27 & 45 & $\mathrm{C}$ & 1.80 & 1.80 & - & \\
\hline & $51 \mathrm{se}$ & BS153 & 1 & 10 & 51 & -71 & 43 & 20 & A & 47.0 & 20.0 & 140 & \\
\hline & 29ne & BS152 & 1 & 10 & 52 & -72 & 42 & 46 & $\mathrm{CA}$ & 0.85 & 0.65 & 160 & \\
\hline
\end{tabular}


TABLE 2-Continued

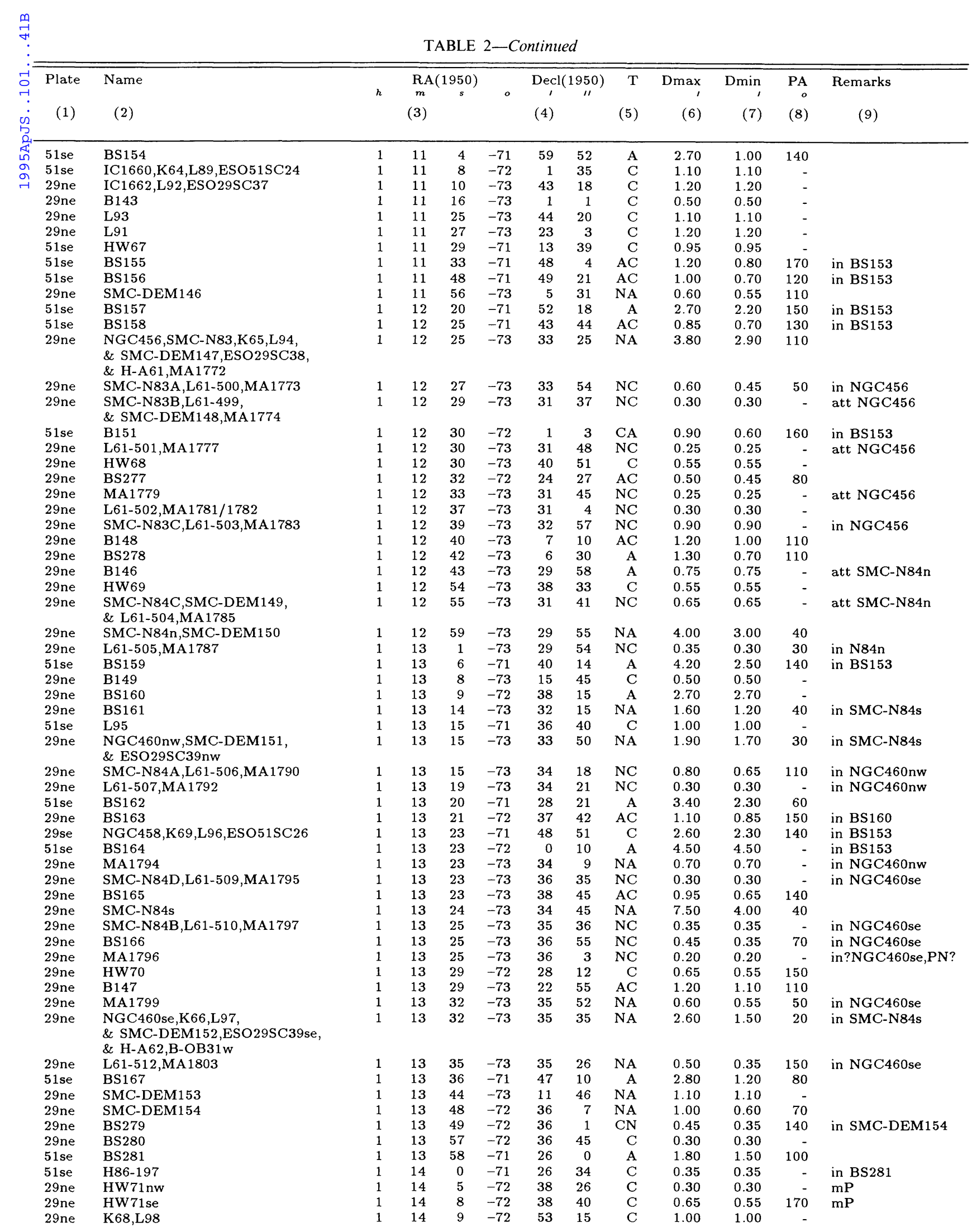


TABLE 2-Continued

\begin{tabular}{|c|c|c|c|c|c|c|c|c|c|c|c|c|c|}
\hline \multicolumn{14}{|c|}{ ABLE 2-Continued } \\
\hline 10. & Plate & Name & & RA & 1950) & & Decl & 1950) & $\mathrm{T}$ & Dmax & Dmin & PA & Remarks \\
\hline in & (1) & (2) & $h$ & $\begin{array}{r}m \\
(3)\end{array}$ & $s$ & 0 & $(4)$ & "' & $(5)$ & & $\begin{array}{r}\prime \\
(7)\end{array}$ & $\begin{array}{r}0 \\
(8)\end{array}$ & (9) \\
\hline 近 & $51 \mathrm{se}$ & BS168 & 1 & 14 & 10 & -72 & 0 & 0 & A & 1.50 & 0.80 & 40 & in $\mathrm{BS} 153$ \\
\hline 'ڤ́' & 29 ne & SMC-DEM 155 & 1 & 14 & 10 & -73 & 27 & 36 & NA & 1.80 & 1.80 & - & in SMC-DEM157 \\
\hline $1 \rightarrow 1$ & 29 ne & HW72 & 1 & 14 & 20 & -73 & 25 & 49 & $\mathrm{CN}$ & 0.55 & 0.45 & 80 & in SMC-DEM157 \\
\hline-- & 29 ne & $\begin{array}{l}\text { NGC } 465, \mathrm{~K} 67, \mathrm{~L} 99, \mathrm{ESO} 29 \mathrm{SC} 40, \\
\& \text { H-A } 63, \mathrm{~B}-\mathrm{OB} 31 \mathrm{e}\end{array}$ & 1 & 14 & 21 & -73 & 35 & 35 & A & 5.00 & 4.00 & 120 & in SMC-DEM157 \\
\hline & 29ne & Sk 157 & 1 & 14 & 28 & -73 & 36 & 29 & $\mathrm{C}$ & 0.60 & 0.60 & - & in NGC465 \\
\hline & 29 ne & Sk 158 & 1 & 14 & 31 & -73 & 34 & 45 & $\mathrm{C}$ & 0.70 & 0.60 & 150 & in NGC465 \\
\hline & 29 se & BS169 & 1 & 14 & 45 & -72 & 1 & 30 & A & 2.30 & 2.30 & - & in $\mathrm{BS} 153$ \\
\hline & 29 ne & SMC-DEM 156 & 1 & 14 & 47 & -73 & 27 & 8 & $\mathrm{NA}$ & 2.80 & 2.60 & 40 & in SMC-DEM 157 \\
\hline & $51 \mathrm{se}$ & HW73 & 1 & 14 & 57 & -71 & 35 & 23 & $\mathrm{C}$ & 1.00 & 0.90 & 60 & \\
\hline & 29 ne & SMC-DEM 157 & 1 & 15 & 0 & -73 & 36 & 0 & NA & 22.0 & 17.0 & 40 & \\
\hline & $51 \mathrm{se}$ & BS170 & 1 & 15 & 15 & -71 & 53 & 11 & A & 1.00 & 1.00 & - & \\
\hline & 29 ne & HW74 & 1 & 15 & 27 & -73 & 25 & 24 & $\mathrm{C}$ & 0.55 & 0.50 & 150 & in SMC-DEM158 \\
\hline & 29 ne & SMC-DEM 158 & 1 & 15 & 28 & -73 & 25 & 8 & NA & 1.80 & 1.50 & 170 & in SMC-DEM 157 \\
\hline & 29 ne & SMC-DEM 159 & 1 & 15 & 38 & -73 & 27 & 54 & $\mathrm{NA}$ & 1.50 & 0.80 & 60 & in SMC-DEM157 \\
\hline & 29 ne & HW75 & 1 & 16 & 11 & -73 & 49 & 56 & $\mathrm{CA}$ & 0.90 & 0.80 & 170 & \\
\hline & $51 \mathrm{se}$ & $\mathrm{L} 100, \mathrm{ESO} 51 \mathrm{SC} 27$ & 1 & 16 & 52 & -72 & 15 & 52 & $\mathrm{C}$ & 1.00 & 1.00 & - & \\
\hline & 29 ne & $\mathrm{B} 156$ & 1 & 18 & 12 & -73 & 21 & 22 & $\mathrm{C}$ & 0.65 & 0.45 & 110 & \\
\hline & 29 ne & HW $77, B 158$ & 1 & 18 & 48 & -72 & 53 & 55 & $\mathrm{C}$ & 1.40 & 1.20 & 50 & \\
\hline & 29 ne & B 155 & 1 & 19 & 9 & -74 & 15 & 38 & $\mathrm{C}$ & 0.55 & 0.55 & - & \\
\hline & 29 ne & BS171 & 1 & 19 & 16 & -72 & 54 & 48 & $\mathrm{AC}$ & 1.10 & 0.85 & 20 & \\
\hline & 29 ne & B159 & 1 & 19 & 32 & -72 & 47 & 40 & A & 2.30 & 2.30 & - & \\
\hline & 29 ne & HW78 & 1 & 20 & 4 & -73 & 21 & 22 & $\mathrm{C}$ & 0.45 & 0.35 & 60 & in $\mathrm{BS} 172$ \\
\hline & 29 ne & BS 172 & 1 & 20 & 5 & -73 & 21 & 34 & A & 2.50 & 1.40 & 50 & \\
\hline & 29 ne & B160 & 1 & 20 & 32 & -73 & 20 & 31 & $\mathrm{~A}$ & 0.80 & 0.70 & 70 & \\
\hline & 29 se & HW79 & 1 & 21 & 43 & -75 & 15 & 45 & $\mathrm{C}$ & 2.70 & 2.70 & - & \\
\hline & 29 ne & HW80 & 1 & 22 & 12 & -73 & 29 & 4 & $\mathrm{C}$ & 0.70 & 0.45 & 60 & $\mathrm{br}^{*}$ in \\
\hline & 29 ne & SMC-DEM160n & 1 & 22 & 23 & -73 & 37 & 26 & NA & 3.10 & 2.70 & 130 & \\
\hline & 29 ne & SMC-DEM160s & 1 & 22 & 29 & -73 & 39 & 36 & NA & 1.40 & 0.80 & 50 & \\
\hline & 29 ne & L101 & 1 & 22 & 31 & -73 & 28 & 2 & A & 2.80 & 1.50 & 0 & \\
\hline & $52 \mathrm{sw}$ & BS173 & 1 & 22 & 32 & -70 & 35 & 23 & $\mathrm{CA}$ & 1.00 & 0.85 & 30 & \\
\hline & 29 ne & $\mathrm{B} 162$ & 1 & 22 & 39 & -73 & 41 & 56 & $\mathrm{CA}$ & 0.55 & 0.40 & 110 & \\
\hline & 29 ne & SMC-N88 & 1 & 22 & 54 & -73 & 24 & 46 & $\mathrm{NC}$ & 0.40 & 0.35 & 120 & in $\mathrm{HW} 81$ \\
\hline & 29 ne & HW81 & 1 & 22 & 56 & -73 & 24 & 52 & $\mathrm{CN}$ & 1.00 & 0.80 & 120 & in SMC-DEM161n \\
\hline & 29 ne & B163 & 1 & 22 & 56 & -73 & 30 & 22 & $\mathrm{AC}$ & 1.20 & 1.20 & - & $\mathrm{b} *$ in,in $\mathrm{H}-\mathrm{A} 65$ \\
\hline & 29 ne & BS174 & 1 & 22 & 57 & -73 & 3 & 46 & A & 1.70 & 1.20 & 110 & \\
\hline & 29 ne & SMC-DEM161s & 1 & 22 & 58 & -73 & 26 & 18 & NA & 1.10 & 0.85 & 40 & in $\mathrm{H}-\mathrm{A} 64$ \\
\hline & 29 ne & SMC-DEM $161 \mathrm{n}$ & 1 & 22 & 59 & -73 & 24 & 58 & $\mathrm{NA}$ & 1.20 & 1.20 & - & in $\mathrm{H}-\mathrm{A} 64$ \\
\hline & 29 ne & BS175 & 1 & 23 & 1 & -73 & 27 & 12 & $\mathrm{~A}$ & 1.50 & 0.75 & 100 & in SMC-DEM163 \\
\hline & 29 ne & $\mathrm{BS} 176$ & 1 & 23 & 2 & -73 & 25 & 36 & $\mathrm{C}$ & 0.50 & 0.50 & - & in $\mathrm{H}-\mathrm{A} 64$ \\
\hline & 29 ne & $\mathrm{H}-\mathrm{A} 65$ & 1 & 23 & 5 & -73 & 29 & 48 & A & 4.50 & 2.20 & 60 & in SMC-DEM 163 \\
\hline & 29 ne & $\mathrm{H}-\mathrm{A} 64$ & 1 & 23 & 13 & -73 & 25 & 1 & $\mathrm{~A}$ & 3.80 & 2.50 & 100 & \\
\hline & 29 ne & HW82 & 1 & 23 & 14 & -73 & 25 & 51 & $\mathrm{C}$ & 0.80 & 0.60 & 40 & in $\mathrm{H}-\mathrm{A} 64$ \\
\hline & 29 ne & BS177 & 1 & 23 & 17 & -73 & 4 & 36 & A & 1.00 & 1.00 & - & \\
\hline & 29 ne & SMC-DEM163 & 1 & 23 & 22 & -73 & 33 & 0 & $\mathrm{NA}$ & 10.0 & 7.00 & 120 & \\
\hline & 29 ne & SMC-DEM162n & 1 & 23 & 26 & -73 & 41 & 48 & $\mathrm{NA}$ & 1.80 & 0.70 & 100 & \\
\hline & 29 ne & SMC-DEM162s & 1 & 23 & 26 & -73 & 42 & 38 & $\mathrm{AN}$ & 3.40 & 1.20 & 130 & \\
\hline & 29 ne & BS 178 & 1 & 23 & 32 & -73 & 34 & 55 & AN & 6.00 & 2.40 & 140 & in SMC-DEM163 \\
\hline & 29 ne & $\mathrm{H} 86-211$ & 1 & 23 & 32 & -73 & 40 & 37 & $\mathrm{C}$ & 0.45 & 0.45 & - & \\
\hline & $52 \mathrm{sw}$ & IC $1708, \mathrm{~L} 102, \mathrm{ESO} 52 \mathrm{SC} 2$ & 1 & 23 & 34 & -71 & 26 & 37 & $\mathrm{C}$ & 1.20 & 1.20 & - & \\
\hline & 29 ne & BS179 & 1 & 23 & 39 & -73 & 41 & 58 & AN & 1.10 & 0.60 & 60 & att SMC-DEM162n \\
\hline & 29 ne & L103,H-A66sw & 1 & 24 & 4 & -73 & 31 & 44 & $\mathrm{~A}$ & 2.70 & 1.90 & 70 & in SMC-DEM163 \\
\hline & 29 ne & $\mathrm{H} 86-212$ & 1 & 24 & 5 & -73 & 45 & 40 & $\mathrm{C}$ & 0.60 & 0.55 & 70 & \\
\hline & 29 ne & BS 180 & 1 & 24 & 8 & -73 & 32 & 12 & NA & 1.10 & 0.85 & 40 & in $\mathrm{L} 103$ \\
\hline & 29 ne & L104 & 1 & 24 & 13 & -73 & 38 & 44 & AN & 1.70 & 1.70 & - & in $\mathrm{SMC}-\mathrm{N} 89$ \\
\hline & 29 ne & H-A 66 ne & 1 & 24 & 16 & -73 & 29 & 13 & A & 2.80 & 2.50 & 20 & in SMC-DEM163 \\
\hline & 29 ne & $\mathrm{BS} 282$ & 1 & 24 & 18 & -73 & 38 & 57 & $\mathrm{C}$ & 0.40 & 0.35 & 90 & in $\mathrm{L} 104$ \\
\hline & 29 ne & SMC-N89,SMC-DEM164,H-A67 & 1 & 24 & 33 & -73 & 38 & 36 & NA & 5.80 & 5.20 & 70 & \\
\hline & 29 ne & BS181 & 1 & 24 & 39 & -73 & 46 & 59 & $\mathrm{~A}$ & 1.10 & 0.90 & 100 & \\
\hline & 29 ne & $\mathrm{BS} 182$ & 1 & 24 & 46 & -73 & 52 & 53 & $\mathrm{~A}$ & 2.50 & 1.60 & 130 & \\
\hline & 29 ne & $\mathrm{BS} 183$ & 1 & 24 & 55 & -74 & 15 & 9 & $\mathrm{~A}$ & 1.70 & 0.70 & 70 & \\
\hline & $52 \mathrm{sw}$ & $\mathrm{B} 168$ & 1 & 25 & 21 & -71 & 2 & 22 & $\mathrm{C}$ & 0.45 & 0.45 & - & \\
\hline & 29 ne & SMC-DEM 165 & 1 & 25 & 26 & -73 & 24 & 47 & NA & 7.00 & 7.00 & - & \\
\hline & 29 ne & $\begin{array}{l}\text { NGC602,SMC-N90,L105, } \\
\text { \& SMC-DEM166,ESO } 29 \text { SC } 43 \text {, } \\
\text { \& H-A68 }\end{array}$ & 1 & 28 & 19 & -73 & 48 & 51 & NA & 3.20 & 2.90 & 90 & in SMC-DEM167 \\
\hline & 29 ne & $\mathrm{B} 164$ & 1 & 28 & 23 & -73 & 47 & 29 & $\mathrm{C}$ & 0.65 & 0.45 & 50 & att NGC602 \\
\hline & $52 \mathrm{sw}$ & BS184 & 1 & 28 & 53 & -71 & 35 & 15 & $\mathrm{CA}$ & 1.40 & 1.40 & - & \\
\hline & $52 \mathrm{sw}$ & BS185 & 1 & 28 & 58 & -71 & 4 & 30 & $\mathrm{~A}$ & 3.80 & 1.80 & 160 & \\
\hline & 29 ne & SMC-DEM167 & 1 & 29 & 12 & -73 & 34 & 30 & NA & 35.0 & 30.0 & 10 & \\
\hline
\end{tabular}


TABLE 2-Continued

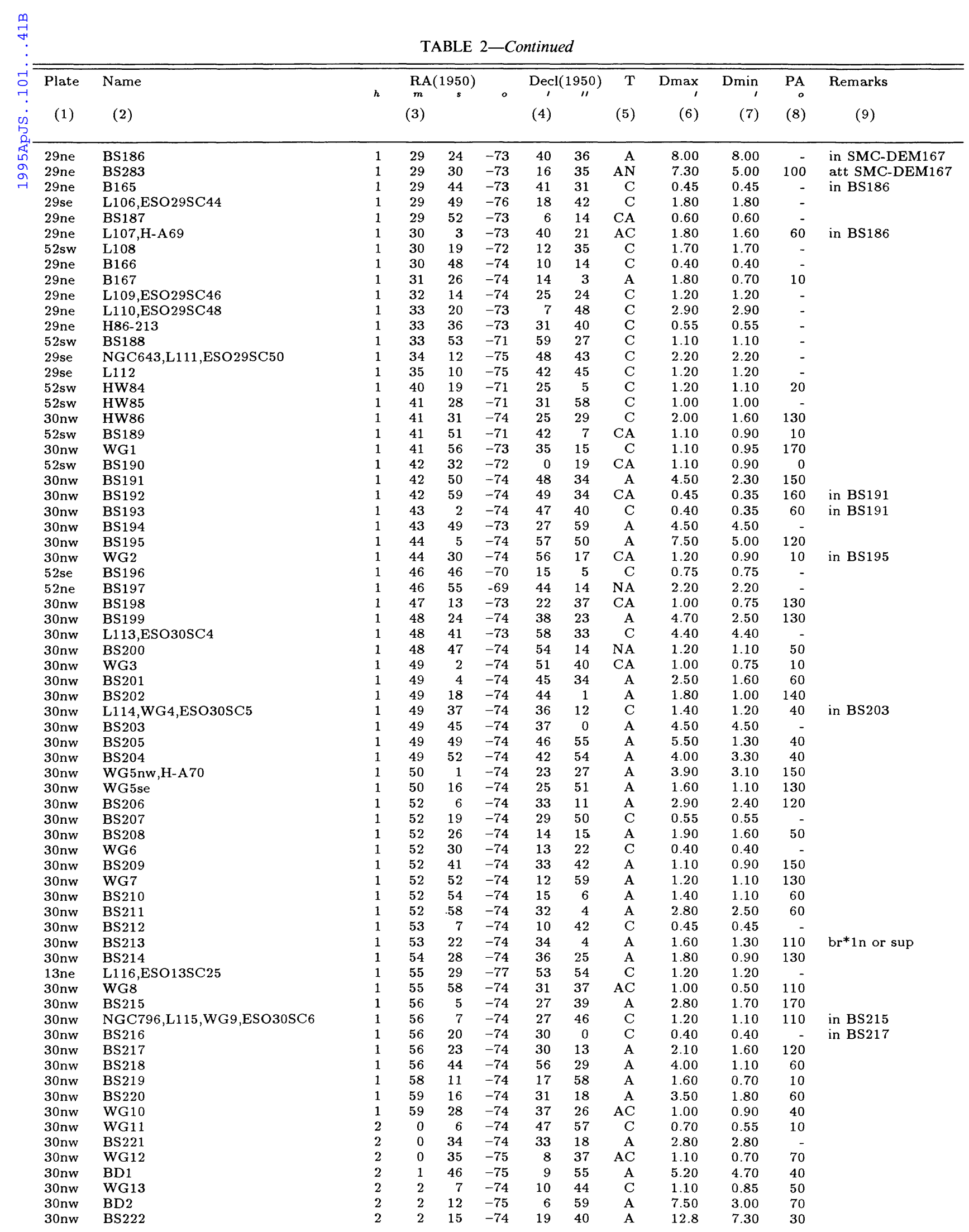


TABLE 2-Continued

\begin{tabular}{|c|c|c|c|c|c|c|c|c|c|c|c|c|}
\hline \multirow{2}{*}{$\begin{array}{l}\text { Plate } \\
\text { (1) }\end{array}$} & \multirow{2}{*}{$\begin{array}{c}\text { Name } \\
\text { (2) }\end{array}$} & \multirow{2}{*}{$h$} & \multicolumn{3}{|c|}{$\mathrm{RA}(1950)$} & \multicolumn{2}{|c|}{$\operatorname{Decl}(1950)$} & \multirow{2}{*}{$\begin{array}{r}\mathrm{T} \\
(5)\end{array}$} & \multirow{2}{*}{$\begin{array}{r}\text { Dmax } \\
(6)\end{array}$} & Dmin & PA & Remarks \\
\hline & & & $\begin{array}{r}m \\
(3)\end{array}$ & $s$ & 0 & (4) & $\prime \prime$ & & & (7) & $\begin{array}{r}0 \\
(8)\end{array}$ & $(9)$ \\
\hline 30nw & BD3 & 2 & 3 & 2 & -75 & 8 & 31 & $\mathrm{~A}$ & 3.40 & 2.90 & 60 & \\
\hline $30 \mathrm{nw}$ & BD4 & 2 & 3 & 27 & -74 & 26 & 34 & A & 5.00 & 3.80 & 20 & br* sup \\
\hline $30 n w$ & BS223 & 2 & 3 & 31 & -74 & 42 & 57 & $\mathrm{C}$ & 0.35 & 0.35 & - & in BS224 \\
\hline $30 \mathrm{nw}$ & BD5 & 2 & 3 & 36 & -74 & 55 & 57 & A & 5.10 & 4.00 & 150 & \\
\hline 30nw & BS224 & 2 & 4 & 0 & -74 & 42 & 13 & A & 4.40 & 4.40 & - & \\
\hline $30 \mathrm{nw}$ & BD6 & 2 & 4 & 1 & -75 & 12 & 50 & A & 5.70 & 3.90 & 100 & \\
\hline $30 n w$ & WG14 & 2 & 4 & 21 & -74 & 45 & 21 & $\mathrm{C}$ & 0.45 & 0.30 & 90 & in $\mathrm{BD} 7$ \\
\hline $30 \mathrm{nw}$ & BD7 & 2 & 4 & 35 & -74 & 44 & 58 & A & 4.00 & 3.00 & 70 & \\
\hline $30 \mathrm{nw}$ & BS225 & 2 & 4 & 38 & -74 & 34 & 20 & A & 1.10 & 0.75 & 40 & \\
\hline $30 n w$ & SMC-DEM 170 & 2 & 5 & 14 & -74 & 38 & 41 & $\mathrm{~N}$ & 1.20 & 0.70 & 90 & in $\mathrm{BD} 9$ \\
\hline $30 \mathrm{nw}$ & BS226 & 2 & 5 & 18 & -74 & 36 & 59 & C & 0.40 & 0.35 & 160 & in $\mathrm{BD} 9$ \\
\hline $30 \mathrm{nw}$ & BD8 & 2 & 5 & 32 & -74 & 51 & 25 & $\mathrm{~A}$ & 5.50 & 2.80 & 70 & \\
\hline $30 \mathrm{nw}$ & BD9 & 2 & 5 & 38 & -74 & 37 & 6 & $\mathrm{~A}$ & 5.50 & 4.00 & 100 & \\
\hline $30 \mathrm{nw}$ & $\mathrm{BS} 227$ & 2 & 5 & 47 & -74 & 24 & 47 & $\mathrm{~A}$ & 1.70 & 0.95 & 110 & in $\mathrm{BD} 10$ \\
\hline $30 \mathrm{nw}$ & BD38 & 2 & 6 & 2 & -73 & 28 & 29 & A & 2.80 & 1.30 & 10 & \\
\hline $30 \mathrm{nw}$ & BD10 & 2 & 6 & 6 & -74 & 23 & 27 & A & 5.60 & 4.80 & 100 & \\
\hline $30 \mathrm{nw}$ & BD11 & 2 & 6 & 11 & -74 & 55 & 36 & A & 3.20 & 2.80 & 70 & \\
\hline $30 \mathrm{nw}$ & BD 12 & 2 & 6 & 41 & -74 & 38 & 53 & $\mathrm{~A}$ & 4.00 & 2.50 & 70 & \\
\hline $30 \mathrm{nw}$ & BD14 & 2 & 6 & 58 & -74 & 54 & 33 & A & 5.00 & 3.00 & 140 & \\
\hline $30 \mathrm{nw}$ & BD13 & 2 & 7 & 4 & -75 & 0 & 56 & A & 4.50 & 2.00 & 60 & in SMC-DEM171 \\
\hline $30 \mathrm{nw}$ & BD 15 & 2 & 7 & 11 & -74 & 8 & 22 & $\mathrm{~A}$ & 5.50 & 2.00 & 60 & \\
\hline $30 n w$ & BD17 & 2 & 7 & 18 & -74 & 44 & 21 & A & 5.80 & 3.60 & 140 & \\
\hline $30 \mathrm{nw}$ & WG15 & 2 & 7 & 18 & -74 & 51 & 45 & $\mathrm{C}$ & 0.35 & 0.25 & 170 & in $\mathrm{BD} 18$ \\
\hline $30 \mathrm{nw}$ & BS228 & 2 & 7 & 24 & -74 & 59 & 55 & $\mathrm{AC}$ & 0.70 & 0.50 & 140 & in $\mathrm{BD} 13$ \\
\hline $30 n w$ & $\mathrm{BD} 18$ & 2 & 7 & 30 & -74 & 52 & 28 & A & 3.20 & 2.30 & 60 & \\
\hline $30 \mathrm{nw}$ & SMC-DEM171 & 2 & 7 & 32 & -74 & 58 & 28 & NA & 7.90 & 7.90 & - & \\
\hline $30 \mathrm{nw}$ & BS229 & 2 & 7 & 34 & -74 & 40 & 27 & $\mathrm{C}$ & 0.45 & 0.35 & 50 & in $\mathrm{BD} 20$ \\
\hline $30 \mathrm{nw}$ & BD16 & 2 & 7 & 36 & -74 & 34 & 8 & $\mathrm{~A}$ & 8.30 & 4.10 & 140 & \\
\hline $30 \mathrm{nw}$ & BS230 & 2 & 7 & 39 & -74 & 58 & 31 & $\mathrm{~A}$ & 1.20 & 0.50 & 130 & in SMC-DEM171 \\
\hline $30 \mathrm{nw}$ & BD20 & 2 & 7 & 45 & -74 & 40 & 47 & A & 4.20 & 2.60 & 90 & \\
\hline $30 \mathrm{nw}$ & WG17 & 2 & 7 & 46 & -74 & 46 & 52 & A & 1.20 & 0.70 & 90 & in $\mathrm{BD} 17$ \\
\hline $30 n w$ & WG16 & 2 & 7 & 46 & -75 & 2 & 9 & $\mathrm{AC}$ & 0.65 & 0.55 & 130 & in SMC-DEM 171 \\
\hline $30 n w$ & BD19 & 2 & 7 & 50 & -74 & 31 & 1 & A & 4.20 & 2.20 & 110 & \\
\hline $30 n w$ & $\mathrm{BD} 22$ & 2 & 8 & 30 & -74 & 49 & 1 & A & 4.90 & 4.50 & 30 & \\
\hline $30 \mathrm{nw}$ & SMC-DEM172 & 2 & 8 & 30 & -75 & 9 & 9 & NA & 4.50 & 3.00 & 50 & \\
\hline $30 \mathrm{nw}$ & BD21 & 2 & 8 & 36 & -74 & 40 & 54 & A & 3.00 & 2.00 & 40 & \\
\hline $30 \mathrm{nw}$ & BD23 & 2 & 8 & 45 & -74 & 32 & 43 & A & 3.20 & 2.50 & 150 & \\
\hline $30 \mathrm{nw}$ & BD25 & 2 & 8 & 52 & -74 & 44 & 53 & A & 3.10 & 2.50 & 100 & \\
\hline $30 \mathrm{nw}$ & BS231 & 2 & 8 & 58 & -74 & 15 & 38 & A & 1.60 & 1.00 & 40 & \\
\hline $30 \mathrm{nw}$ & BS232 & 2 & 9 & 1 & -74 & 15 & 21 & $\mathrm{CA}$ & 0.40 & 0.40 & - & in $\mathrm{BS} 231$ \\
\hline $30 \mathrm{nw}$ & BD24 & 2 & 9 & 2 & -74 & 37 & 4 & A & 4.50 & 2.00 & 130 & \\
\hline $30 \mathrm{nw}$ & BD26 & 2 & 9 & 7 & -74 & 31 & 15 & A & 3.00 & 2.50 & 50 & \\
\hline $30 \mathrm{nw}$ & BD28 & 2 & 9 & 21 & -74 & 34 & 3 & A & 3.00 & 2.40 & 40 & \\
\hline $30 \mathrm{nw}$ & BD27 & 2 & 9 & 29 & -74 & 45 & 30 & A & 3.90 & 2.50 & 30 & \\
\hline $30 \mathrm{nw}$ & BD29 & 2 & 9 & 33 & -74 & 41 & 20 & A & 3.60 & 2.90 & 150 & \\
\hline $30 \mathrm{nw}$ & BS233 & 2 & 10 & 18 & -74 & 23 & 19 & $\mathrm{AC}$ & 1.20 & 1.10 & 130 & in SMC-DEM169 \\
\hline $30 \mathrm{nw}$ & SMC-DEM169 & 2 & 10 & 42 & -74 & 24 & 0 & NA & 9.50 & 2.10 & 130 & \\
\hline $30 \mathrm{nw}$ & BS234 & 2 & 10 & 54 & -74 & 30 & 45 & $\mathrm{AC}$ & 0.90 & 0.90 & - & \\
\hline $30 \mathrm{nw}$ & BS235 & 2 & 11 & 26 & -74 & 21 & 19 & C & 0.30 & 0.25 & 140 & \\
\hline $30 n w$ & BD30 & 2 & 11 & 56 & -74 & 18 & 36 & A & 4.10 & 2.30 & 150 & \\
\hline $30 \mathrm{nw}$ & SMC-DEM168 & 2 & 12 & 0 & -74 & 25 & 0 & NA & 7.00 & 4.00 & 40 & \\
\hline 30 ne & BS236 & 2 & 12 & 46 & -74 & 17 & 51 & A & 2.80 & 2.20 & 40 & \\
\hline 30ne & BD31 & 2 & 13 & 33 & -74 & 21 & 58 & $\mathrm{~A}$ & 5.20 & 2.50 & 110 & \\
\hline 30 ne & BD32 & 2 & 13 & 33 & -74 & 25 & 4 & A & 4.60 & 2.10 & 90 & \\
\hline $30 \mathrm{nw}$ & BS237 & 2 & 13 & 46 & -74 & 18 & 44 & A & 0.65 & 0.50 & 90 & in $\mathrm{BD} 30$ \\
\hline 30 ne & BD33 & 2 & 13 & 56 & -74 & 17 & 15 & A & 5.00 & 2.90 & 100 & \\
\hline 30ne & BS238 & 2 & 13 & 57 & -74 & 18 & 7 & $\mathbf{A}$ & 0.90 & 0.80 & 120 & in $\mathrm{BD} 33$ \\
\hline 30ne & BS239 & 2 & 14 & 14 & -74 & 12 & 46 & A & 1.20 & 0.65 & 10 & in BD34 \\
\hline 30 ne & BD34 & 2 & 14 & 15 & -74 & 12 & 34 & A & 4.70 & 3.60 & 60 & \\
\hline 30ne & BS240 & 2 & 14 & 31 & -74 & 11 & 6 & $\mathrm{C}$ & 0.35 & 0.35 & - & in $\mathrm{BD} 34$ \\
\hline 30ne & WG18 & 2 & 15 & 19 & -74 & 16 & 8 & A & 1.20 & 1.00 & 10 & \\
\hline 30ne & BD35 & 2 & 15 & 33 & -74 & 45 & 14 & A & 4.50 & 2.00 & 70 & \\
\hline 30ne & BD36 & 2 & 16 & 52 & -74 & 27 & 50 & A & 2.60 & 2.60 & - & \\
\hline 30ne & BD37 & 2 & 17 & 27 & -74 & 38 & 11 & A & 5.00 & 5.00 & - & \\
\hline 30 ne & BS241 & 2 & 17 & 43 & -74 & 32 & 11 & A & 1.70 & 1.70 & - & \\
\hline 30ne & BS242 & 2 & 17 & 49 & -74 & 26 & 49 & A & 3.20 & 3.20 & - & \\
\hline 30 ne & BS243 & 2 & 19 & 10 & -74 & 25 & 40 & A & 3.00 & 1.80 & 140 & \\
\hline 30 ne & BD41 & 2 & 19 & 53 & -73 & 24 & 48 & A & 3.30 & 1.30 & 160 & \\
\hline 30 ne & IDK 1 & 2 & 20 & 25 & -73 & 20 & 0 & A & 4.70 & 2.90 & 90 & \\
\hline 30ne & BD42 & 2 & 24 & 8 & -73 & 16 & 0 & A & 2.80 & 2.20 & 0 & \\
\hline
\end{tabular}


TABLE 2-Continued

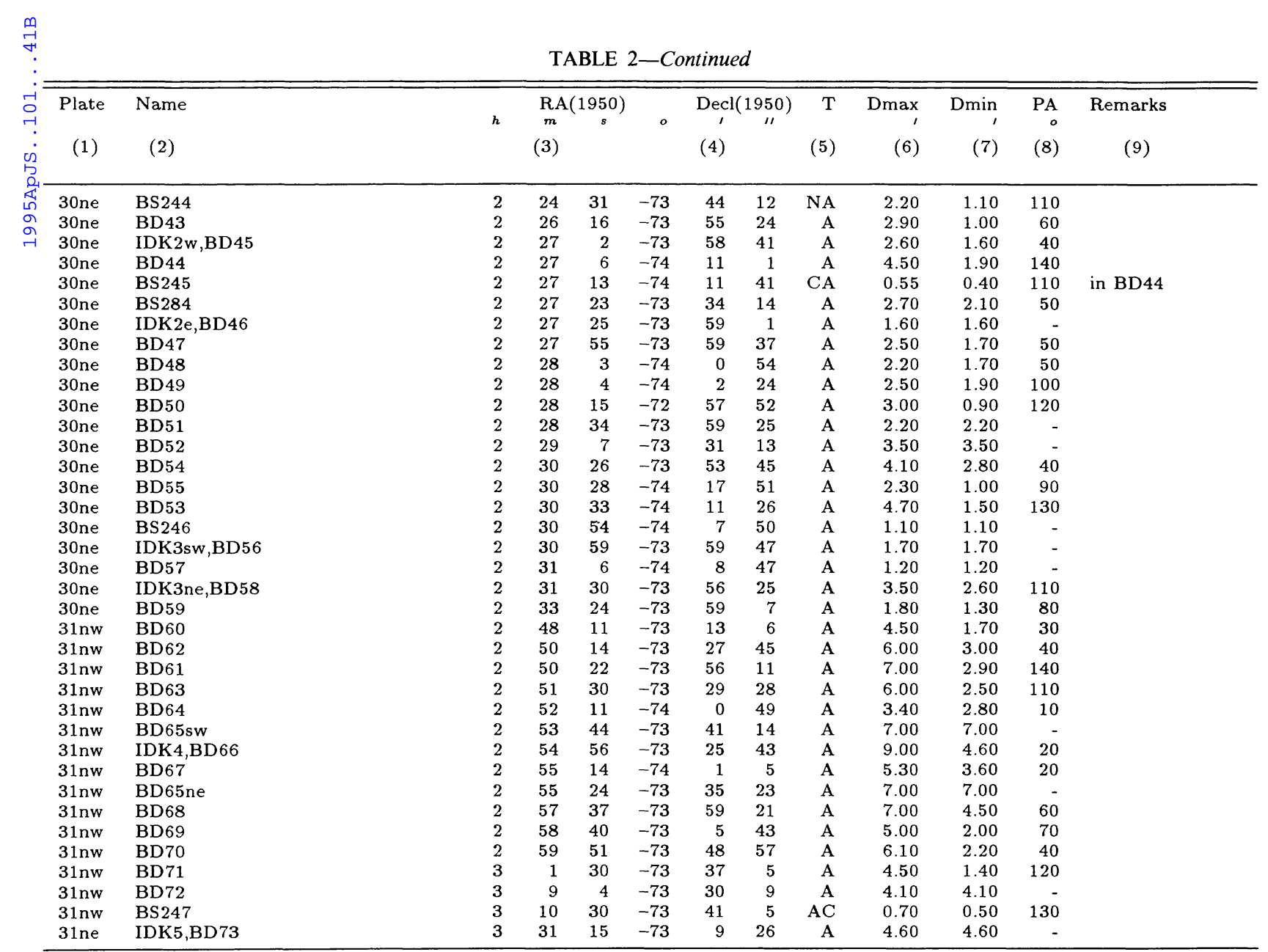


TABLE 3

EXCLUDED ENTRIES

\begin{tabular}{|c|c|c|c|}
\hline Name & Remarks & Name & Remarks \\
\hline SMC-N1,L61-2 & stellar,in B3 & MA163 & stellar \\
\hline H86-1 & not clear & H86-95 & not clear \\
\hline H86-4 & not clear & H86-96 & not clear \\
\hline B5 & 2 stars & MA193 & stellar, in H86-97 \\
\hline H86-5 & not clear & S10,L61-107a,MA219 & stellar \\
\hline H86-7 & not clear & MA218 & stellar,in NGC269 \\
\hline H86-8 & not clear & SMC-DEM41 & cluster NGC269 \\
\hline H86-9 & not clear & MA226 & stellar,in N27 \\
\hline H86-12 & not clear & SMC-N29,L61-115,MA241 & stellar,PN \\
\hline H86-14 & not clear & B51 & asterism \\
\hline SMC-N2,L61-14 & stellar,PN & B46 & asterism? \\
\hline $\mathrm{H} 86-17$ & gal+star? & SMC-N31,L61-249,MA249 & stel,PN,in?sup?H-A16 \\
\hline H86-18 & not clear & MA272 & stellar,in B50 \\
\hline H86-19 & not clear & MA277 & stellar,in B50 \\
\hline HW 7 & galaxy? & MA290 & stellar,PN \\
\hline H86-26 & not clear & MA291 & stellar,PN \\
\hline B7 & asterism? & SMC-N38,L61-144,MA325 & stellar,PN \\
\hline SMC-N4,L61-16 & stellar,PN & SMC-N39,L61-151,MA364 & stellar \\
\hline B8 & asterism & S11,L61-153,MA366 & stellar \\
\hline H86-34 & not clear & SMC-N40,L61-134,MA371 & stellar,PN \\
\hline H86-37 & not clear & S12,L61-164,MA392 & stellar \\
\hline H86-39 & not clear & L61-161, MA389 & stellar \\
\hline $\mathrm{B} 13$ & not clear & B58 & asterism? \\
\hline H86-44 & not clear & MA406 & stellar,PN \\
\hline $\mathrm{H} 86-45$ & not clear & SMC-N43,L61-174,MA433 & stellar,PN \\
\hline H86-46 & not clear & H $86-118$ & not clear \\
\hline $\mathrm{H}-\mathrm{A} 2$ & Galactic sts? & S13,MA450 & stellar \\
\hline H86-49 & not clear & SMC-N42,L61-179,MA467 & stellar,PN \\
\hline B11 & asterism? & MA482 & stellar,PN \\
\hline $\mathrm{H} 86-50$ & not clear & B63 & asterism \\
\hline H86-51 & not clear & MA491 & stellar,PN \\
\hline $\mathrm{B} 17$ & asterism? & MA492 & stellar,in N45 \\
\hline MA11 & stellar,in NGC220 & S14,MA502 & stellar \\
\hline SMC-DEM3 & cluster NGC220 & SMC-N44,L61-191,MA512 & stellar,PN \\
\hline MA14 & stellar,PN & SMC-DEM61 & cluster K29 \\
\hline MA19 & stellar,in B19 & MA509 & stellar,in $\mathrm{K} 29$ \\
\hline SMC-DEM4 & cluster $\mathrm{K} 17$ & L61-200,MA518 & stellar,PN \\
\hline MA22 & stellar,PN & SMC-N47,L61-196,MA519 & stellar,PN \\
\hline $\mathrm{H} 86-61$ & not clear & MA549 & stellar, in H86-129 \\
\hline SMC-N5,L61-32,MA23 & stellar,PN & MA543 & stellar,in B62 \\
\hline SMC-N6,L61-33,MA29 & stellar,PN & MA555 & stellar, in B56a \\
\hline H86-63 & not clear & $\mathrm{H} 86-131$ & $=\mathrm{H} 86-128$ \\
\hline H86-64 & not clear & L61-202,MA558 & stellar, in $\mathrm{H} 86-130$ \\
\hline H86-65 & galaxy? & S16,L61-206,MA574 & stellar,in K30 \\
\hline H86-66 & galaxy? & MA575 & stellar,in K30 \\
\hline H86-67 & not clear & MA586 & stellar,in K30 \\
\hline HW17 & galaxy & L61-209,MA588 & stellar,in K30 \\
\hline MA39 & stellar,PN & H86-137 & $=\mathrm{H} 86-133$ \\
\hline H86-69 & not clear & L61-220,MA616 & stellar,in K31 \\
\hline SMC-DEM8 & cluster L28 & MA614 & stellar,in DEM73 \\
\hline MA44 & stellar,PN & MA633 & stellar,in B69 \\
\hline B32 & asterism & MA647 & stellar,in B69 \\
\hline S2,MA46 & stellar? & L61-239,MA652 & stellar,PN \\
\hline SMC-N8,L61-41,MA47 & stellar, $\mathrm{PN}$ & MA662 & stellar,in B71 \\
\hline SMC-N7,L61-43,MA49 & stellar,PN & SMC-DEM75 & cluster L48 \\
\hline S3,L61-48,MA55 & stellar, in NGC242 & MA668 & stellar,in NGC299 \\
\hline H $86-71$ & not clear & MA671 & stellar? \\
\hline B30 & gal+sts? & MA689 & stellar,in H86-145 \\
\hline L61-49,MA61 & stellar,PN & MA690 & stellar,in BS67a \\
\hline MA73 & stellar,PN & MA691 & stellar,in BS67a \\
\hline $\mathrm{H} 86-73$ & galaxy? & S17,L61-249,MA687 & stellar \\
\hline L61-66,MA98 & stellar,PN & MA700 & stellar,PN \\
\hline L61-71,MA104 & stellar, $\mathrm{PN}$, in $\mathrm{B} 41$ & SMC-N53,L61-247,MA 740 & stellar \\
\hline MA111 & small part of $\mathrm{N} 12 \mathrm{~B}$ & H86-141 & star? \\
\hline MA112 & stellar?,in NGC249 & MA744 & stellar,in NGC306 \\
\hline $\mathrm{H} 86-75$ & not clear & MA745 & stellar,in NGC306 \\
\hline $\mathrm{L} 61-78$ & stellar,in NGC261 & MA747 & stellar,in NGC306 \\
\hline H86-81 & not clear & S19,L61-254,MA751 & stellar \\
\hline $\mathrm{H} 86-82$ & galaxy? & MA762 & stellar,in BA72a \\
\hline SMC-DEM26 & cluster HW22 & H86-153 & not clear \\
\hline SMC-N18,L61-83,MA152 & stellar,PN & MA790 & stellar,in B78 \\
\hline
\end{tabular}


TABLE 3-Continued

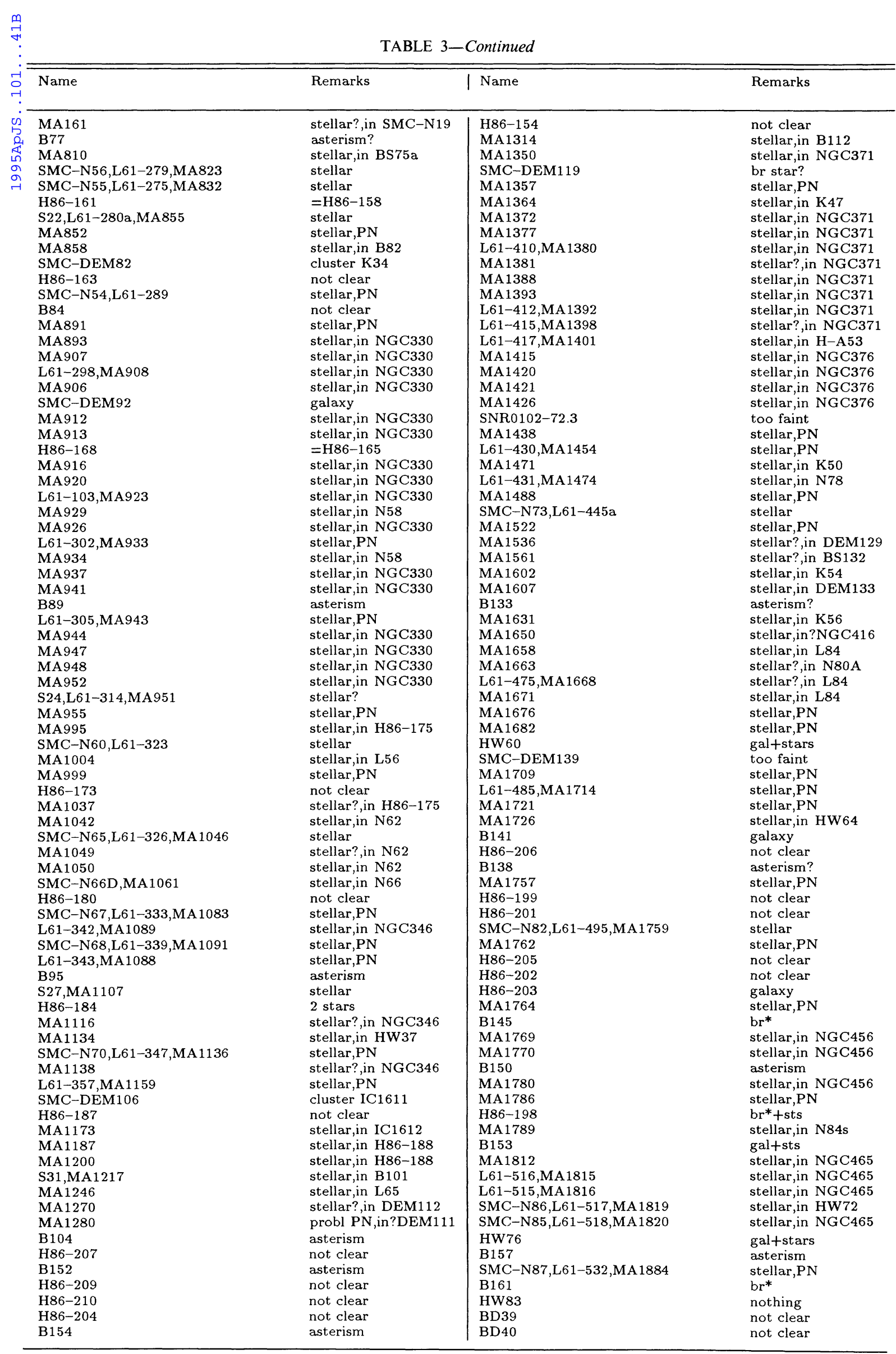




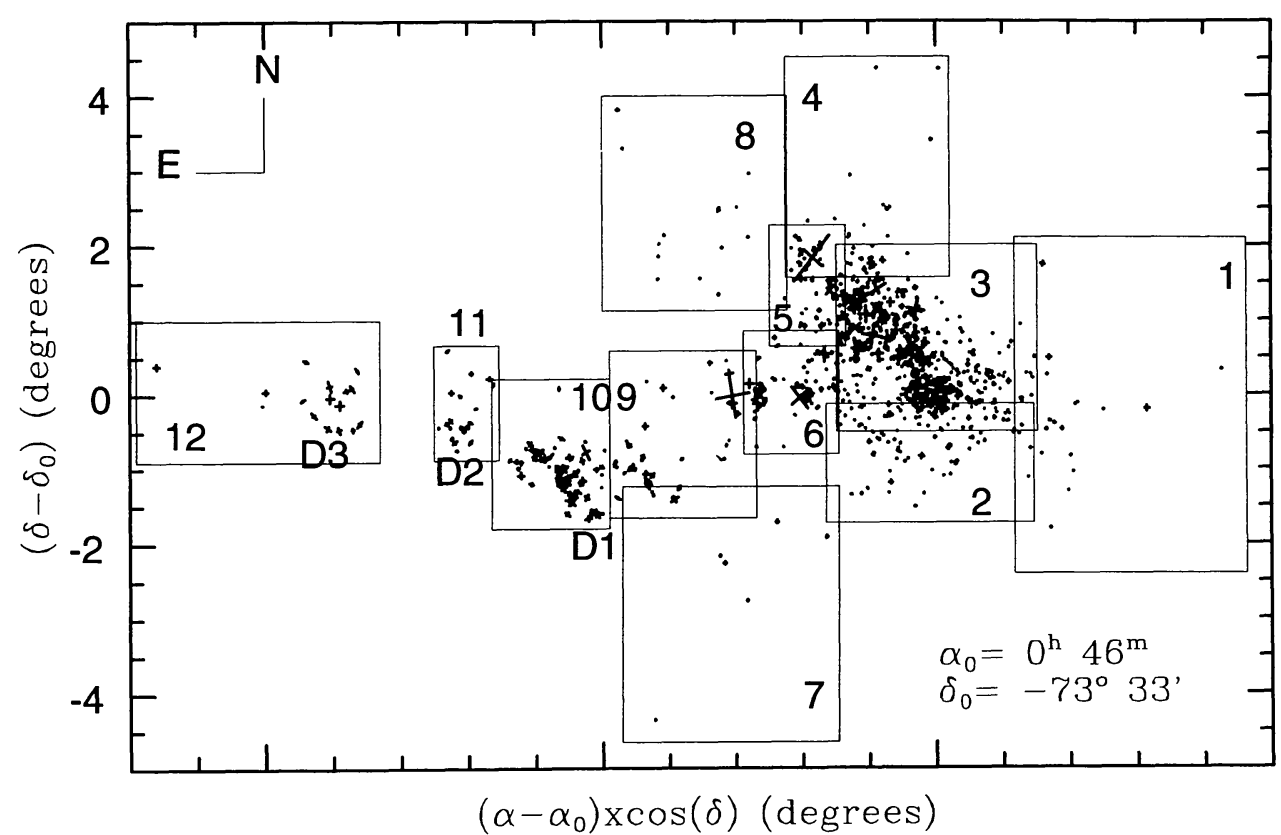

FIG. $1 a$

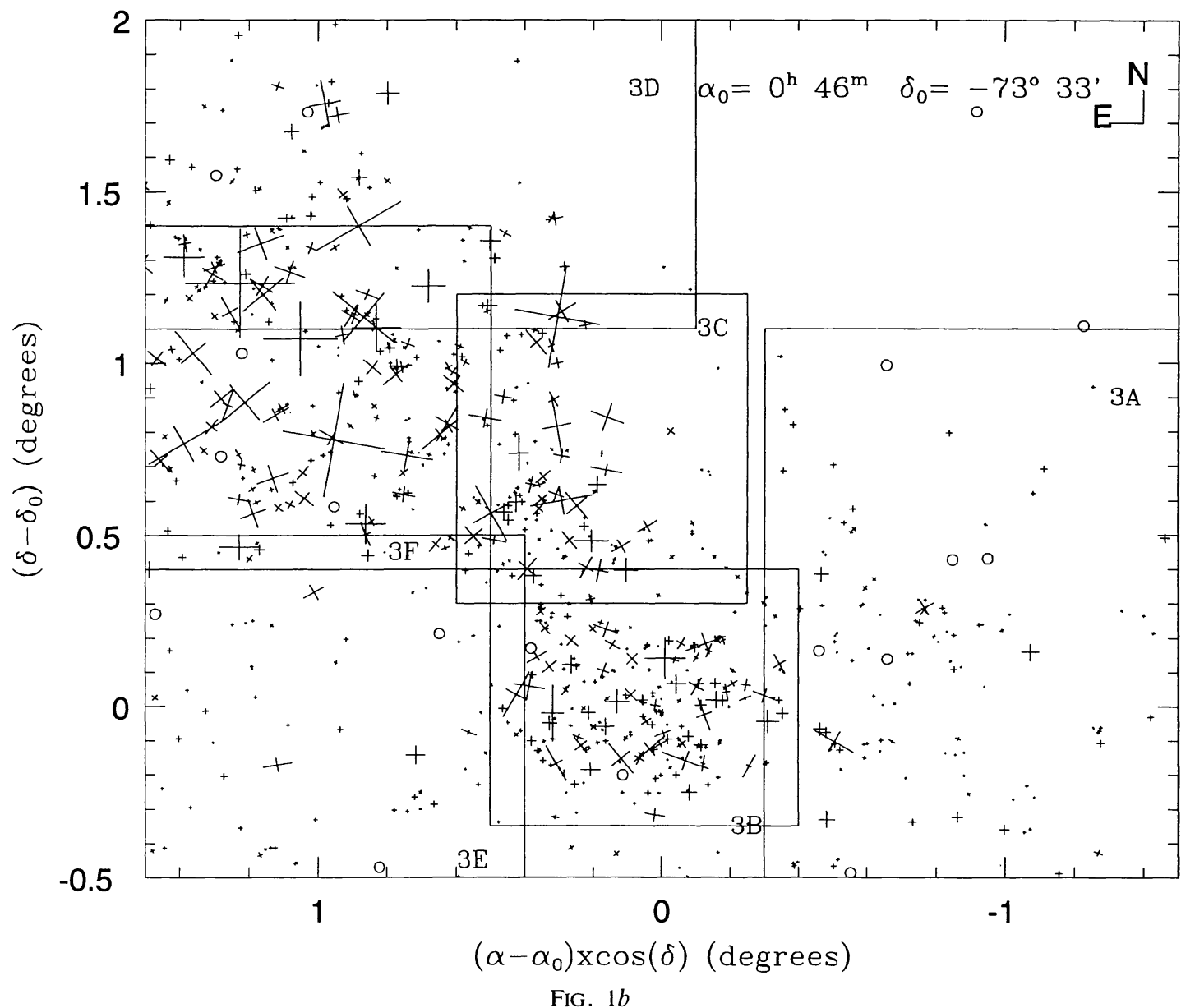

FIG. 1.-Arrangement of the charts. (a) SMC (right) and inter-Cloud region (left); $(b)$ enlargement of the SMC central region; numbers identify the charts. 


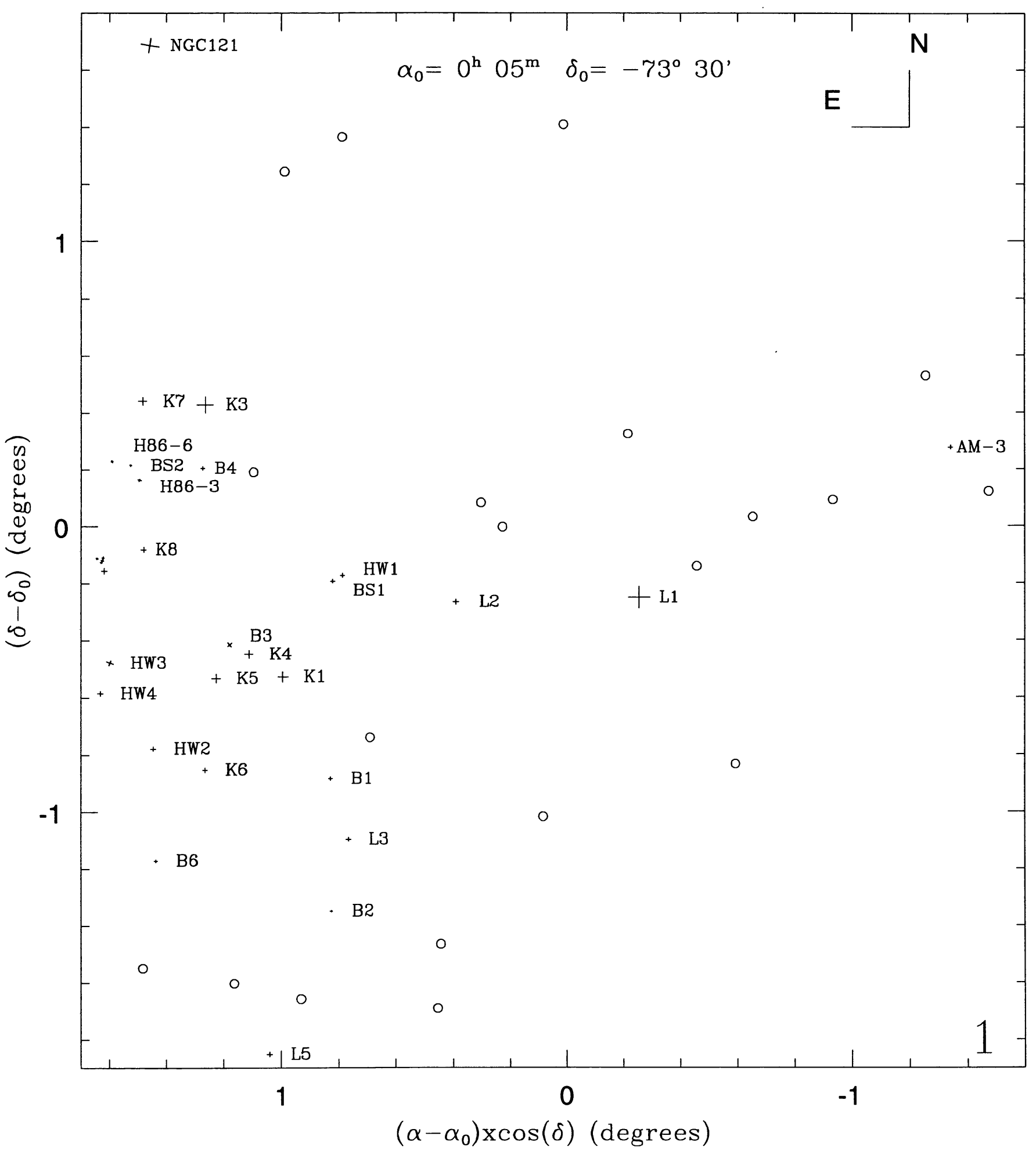

FIG. $2 a$

FIG. 2.- $(a-s)$ Finding charts; each figure shows the right ascension and declination $\left(\alpha_{0}, \delta_{0}\right)$ of the origin of the scale in degrees which is provided in the axes. North and east are also indicated. Objects are plotted to scale and according to position angle; labels are in general to the right (west) of the object. Circles are SAO stars for reference. 


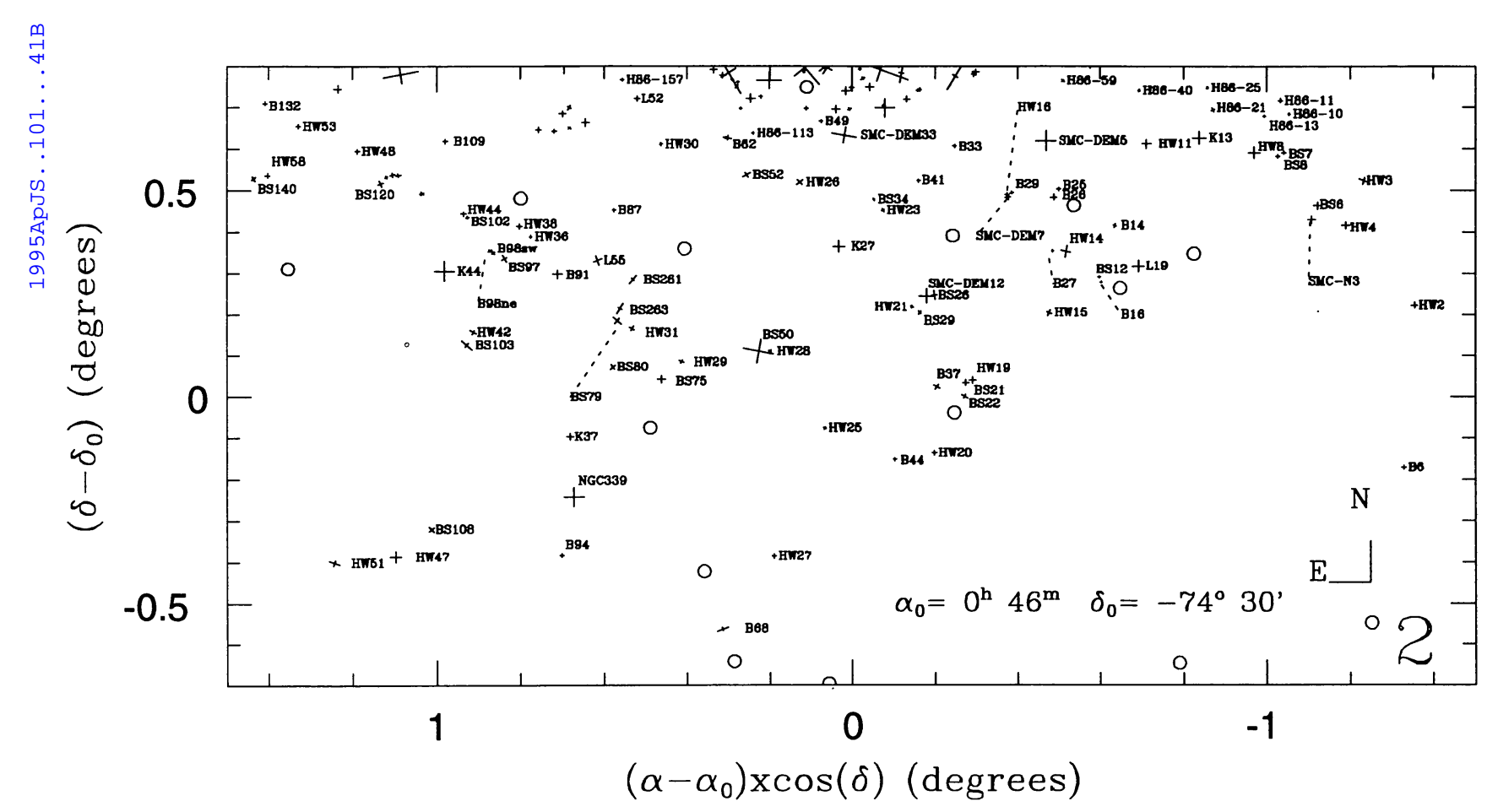

FIG. $2 b$ 


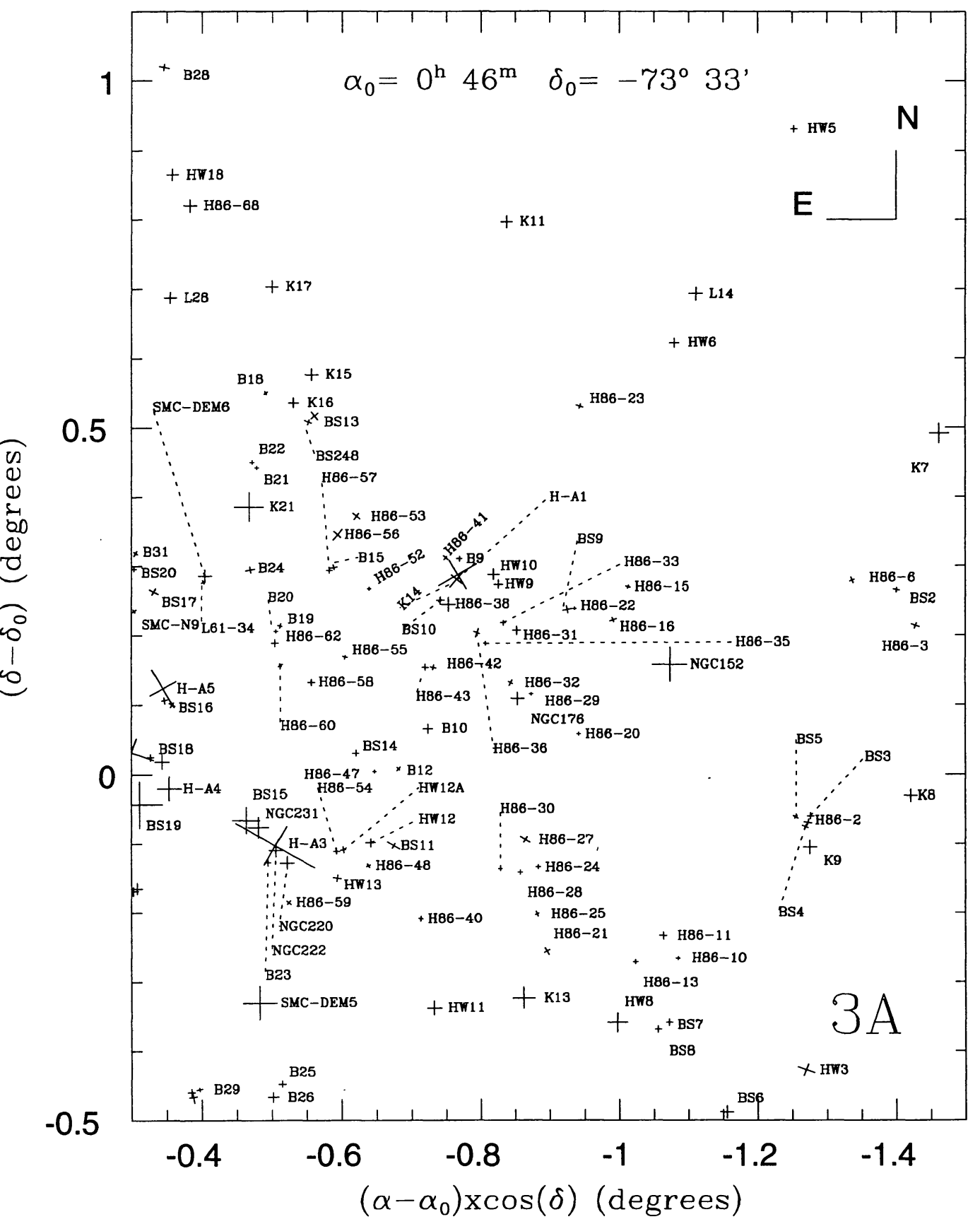

FIG. $2 c$ 


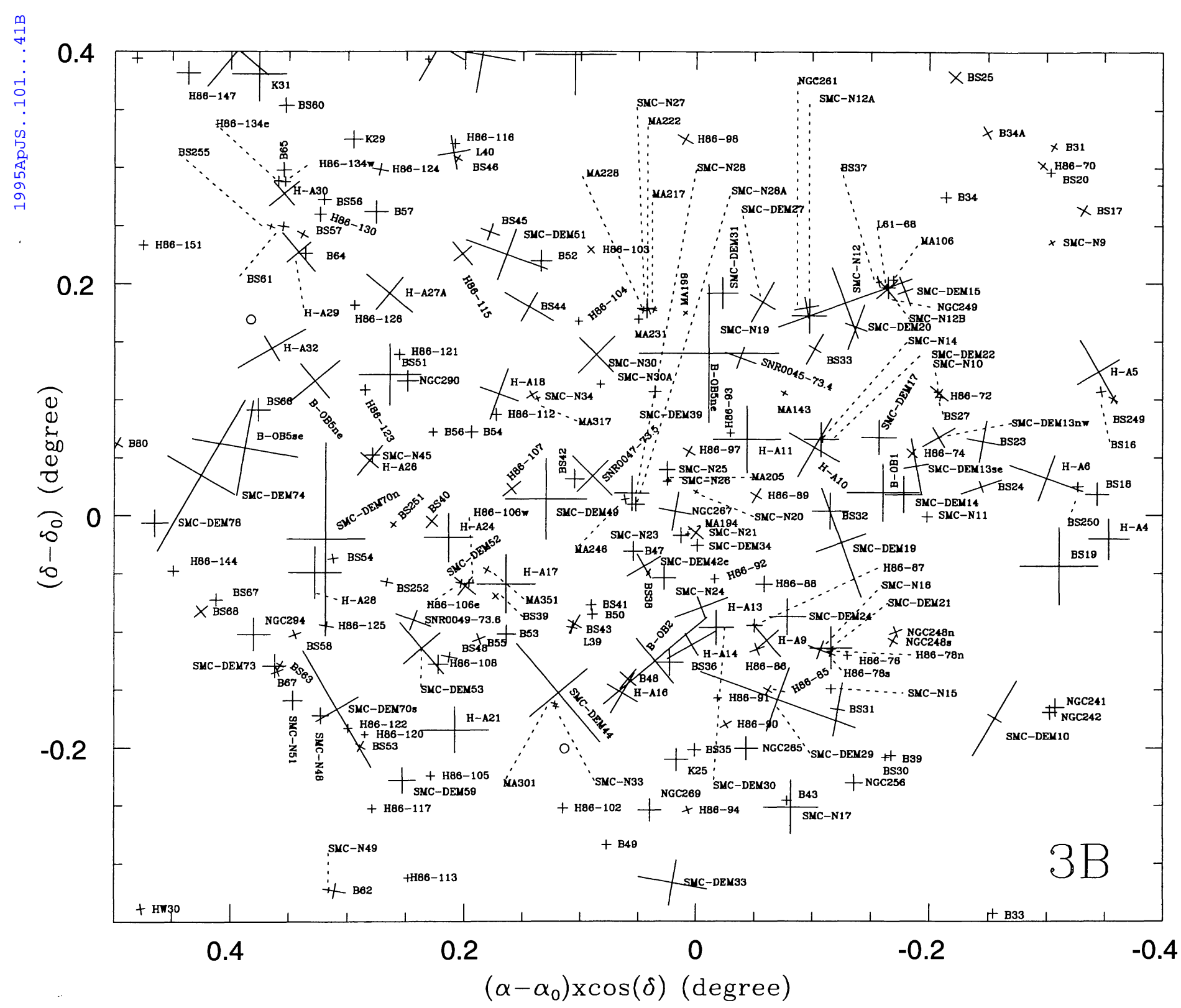

FIG. $2 d$ 
1.2
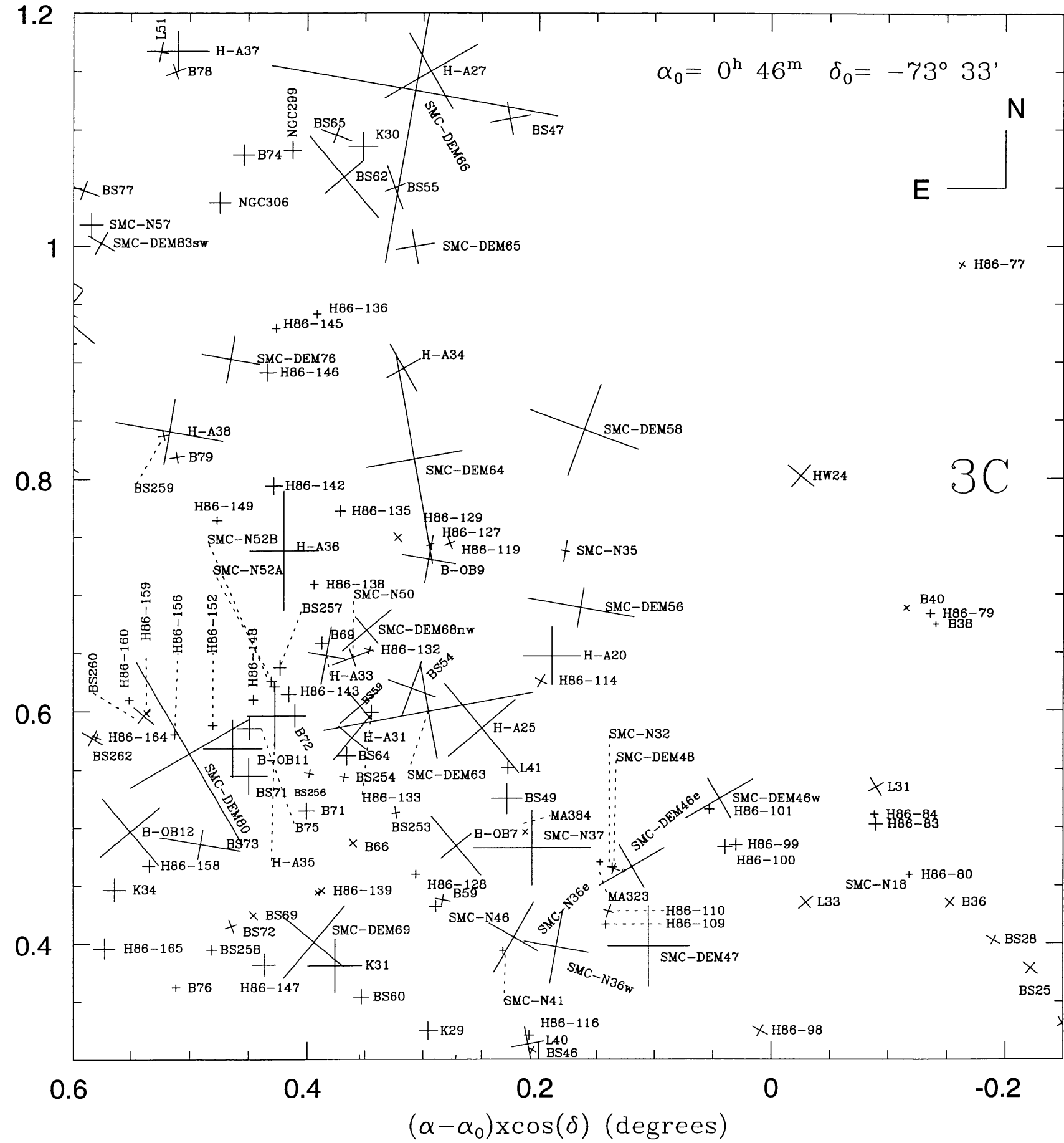

FIG. $2 e$ 


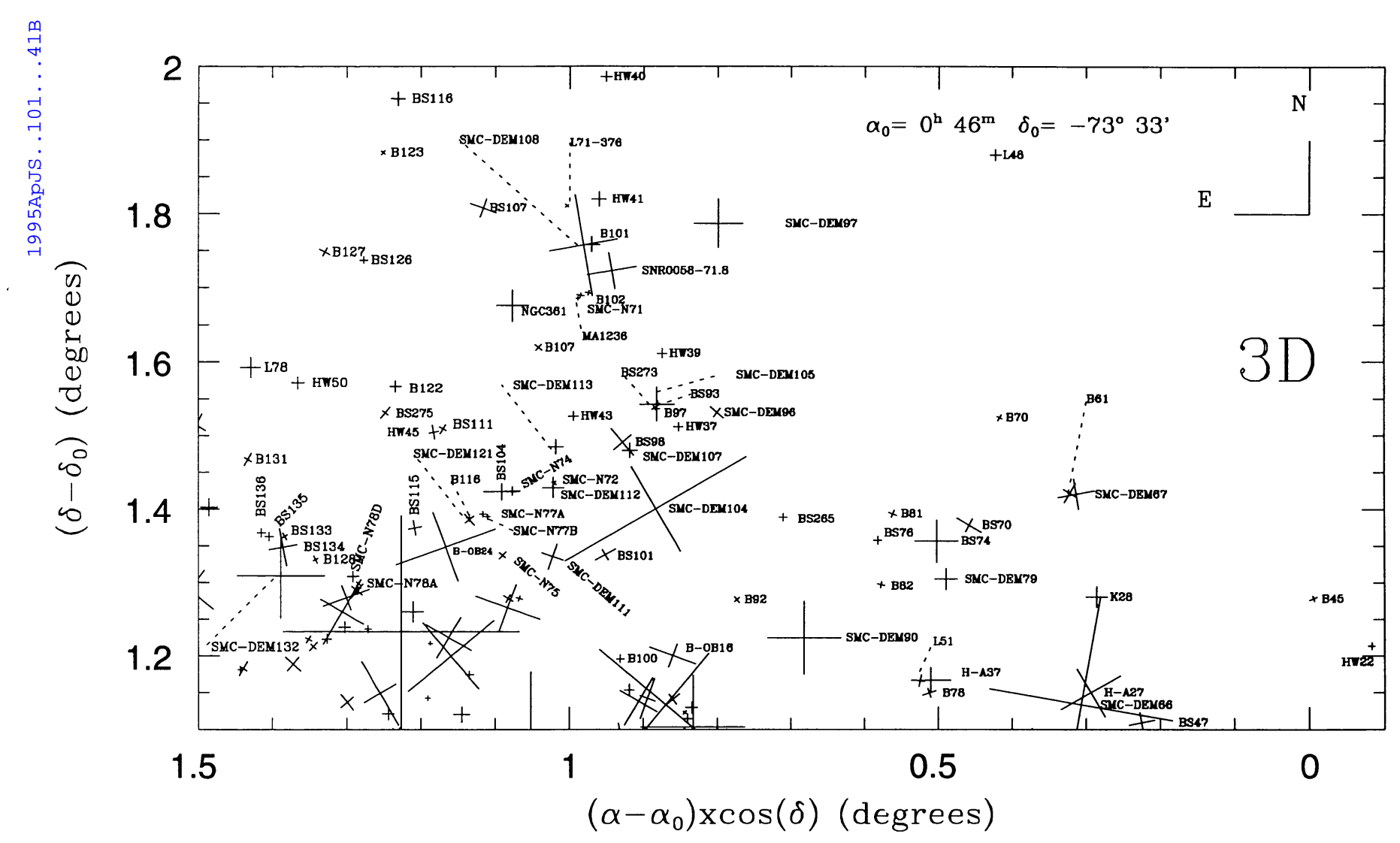

FIG. $2 f$ 


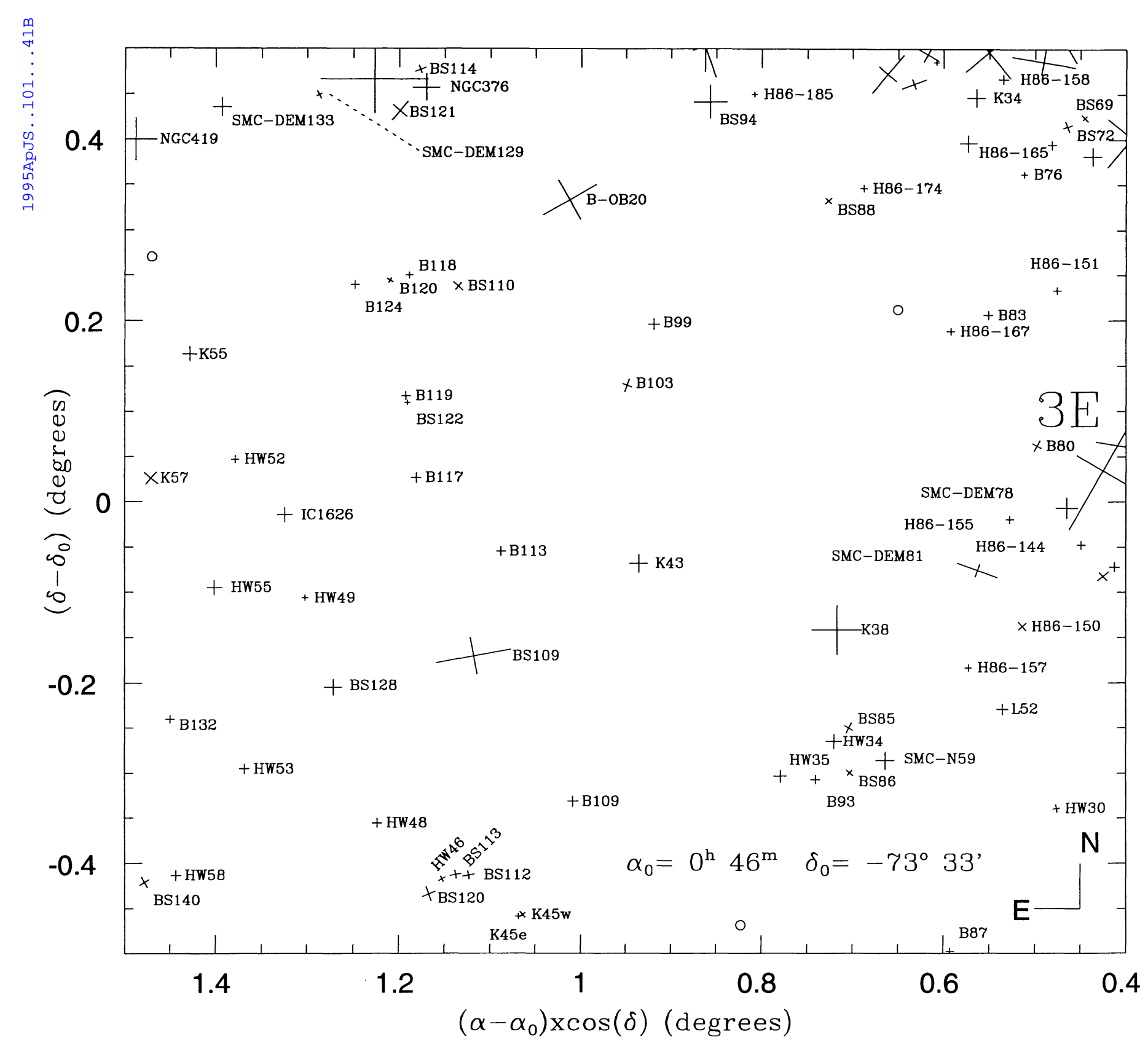

FIG. $2 g$ 


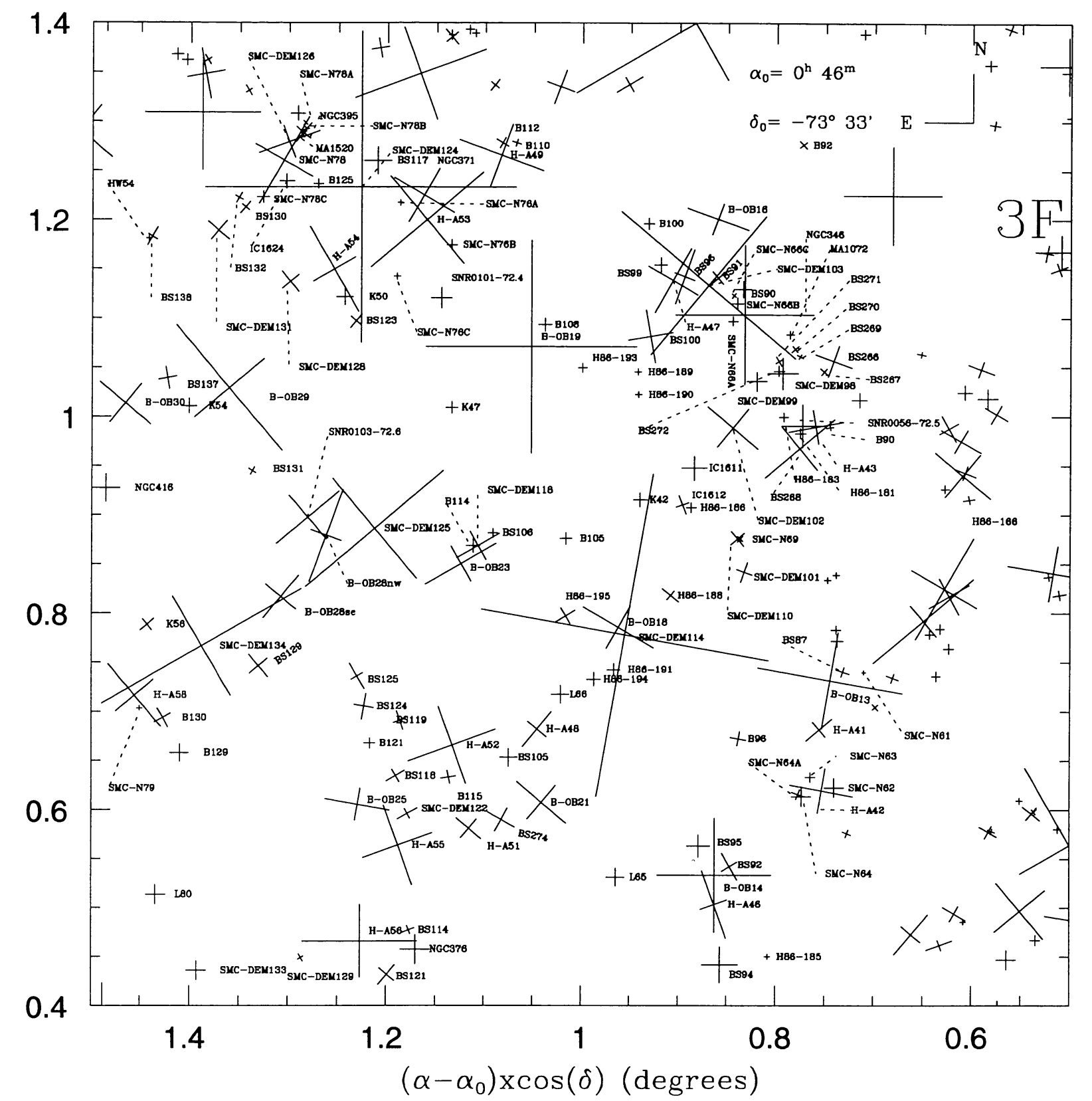

FIG. $2 h$ 


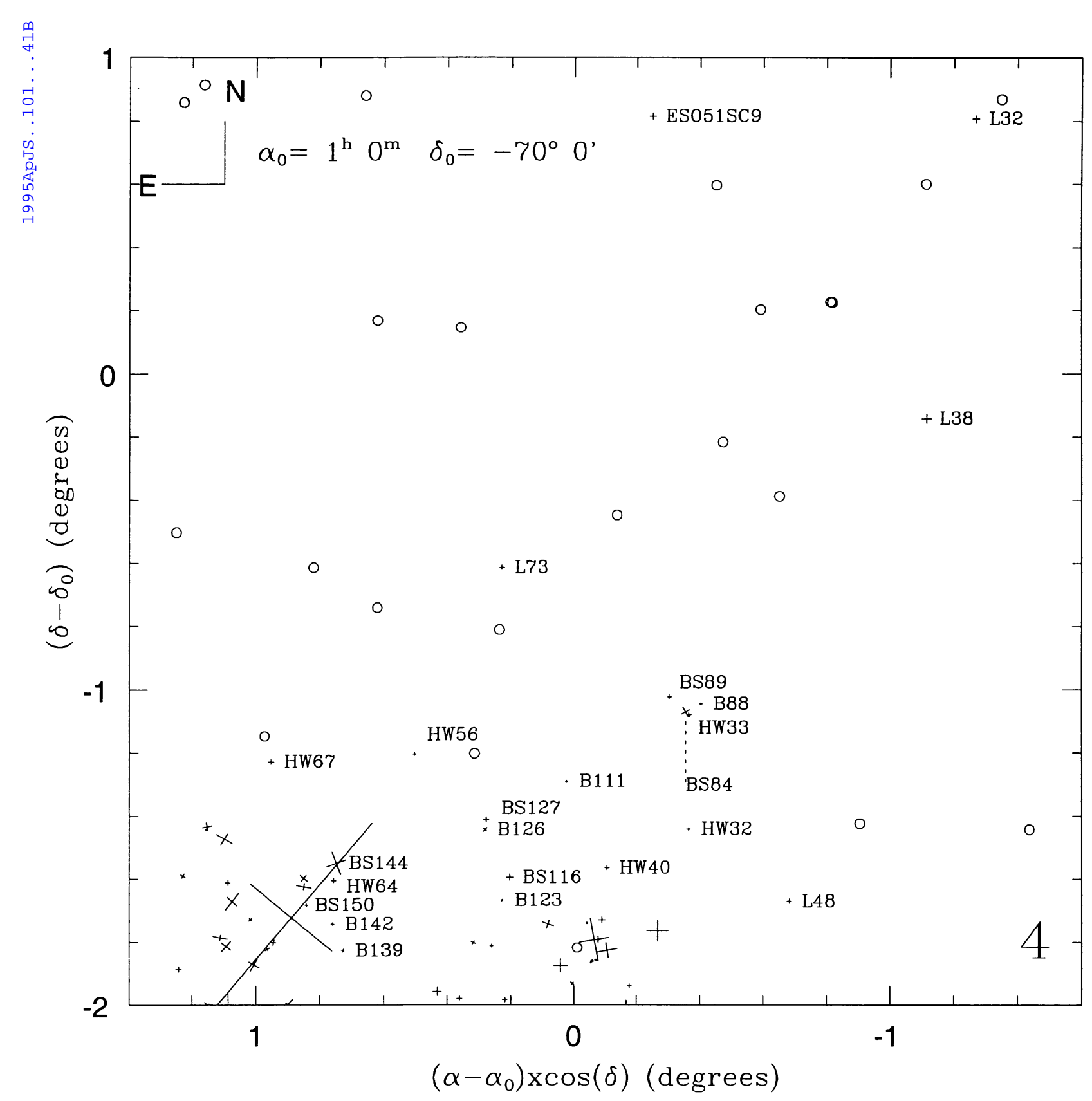

FIG. $2 i$ 


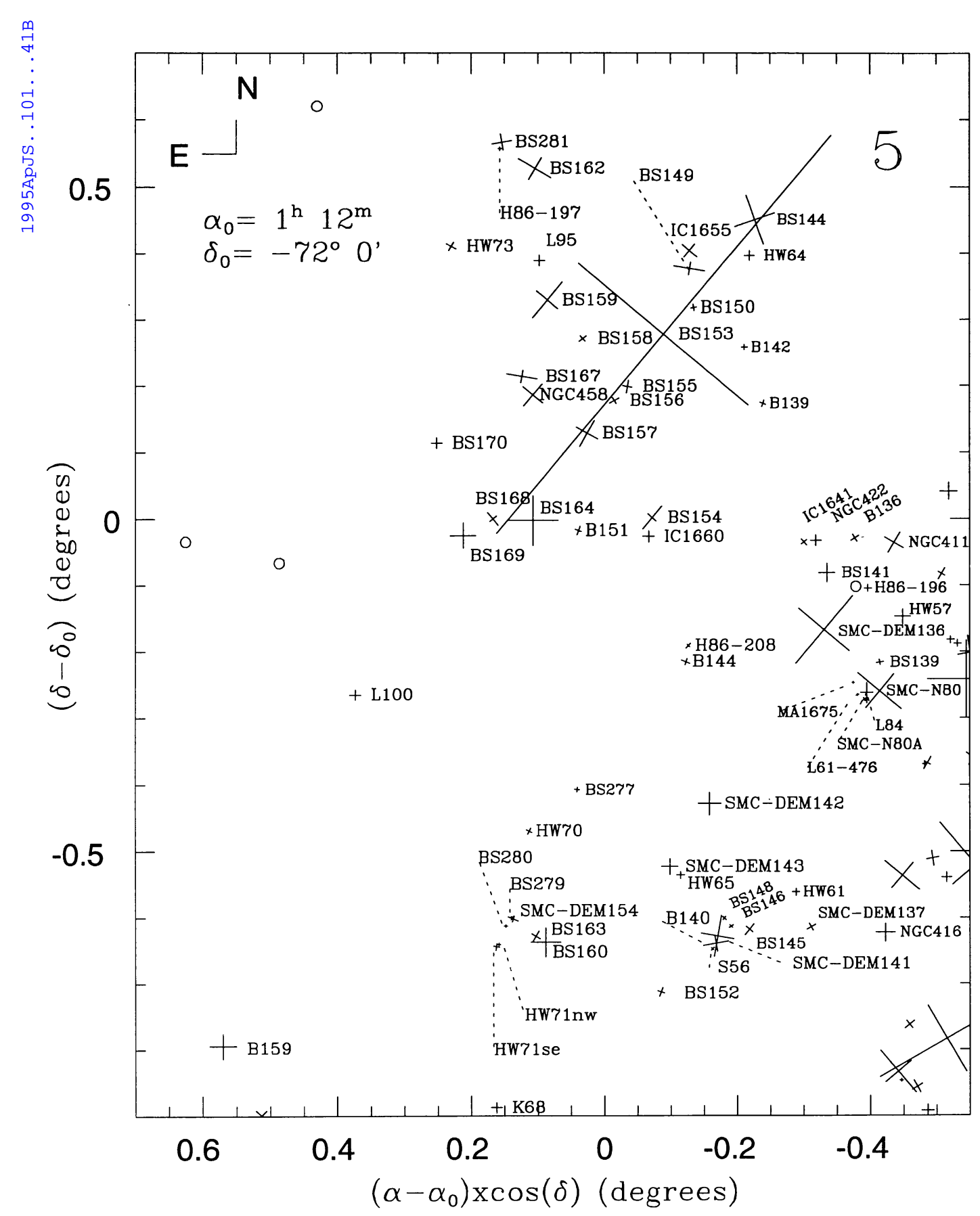

FIG. $2 j$ 


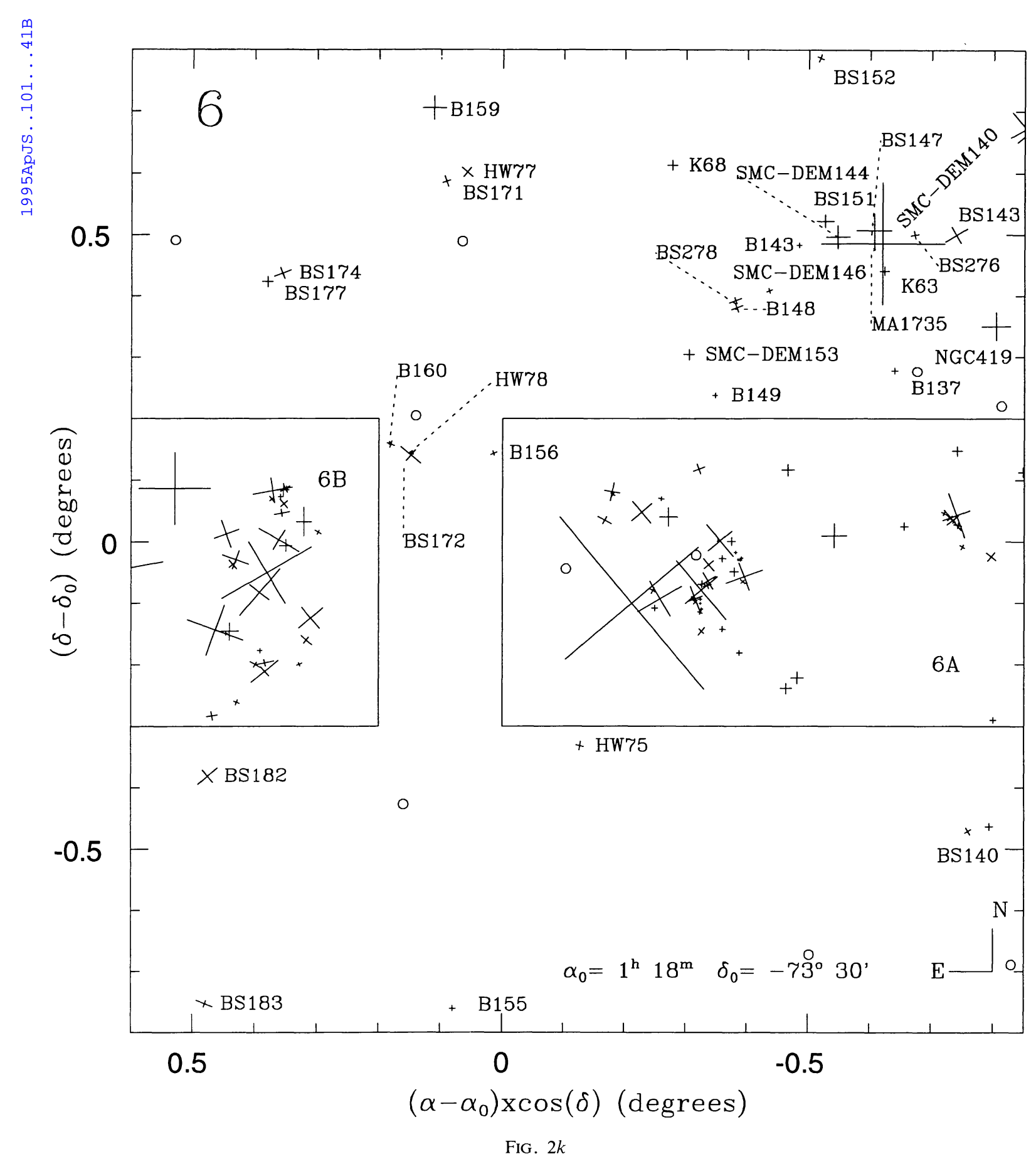

FIG. $2 k$ 


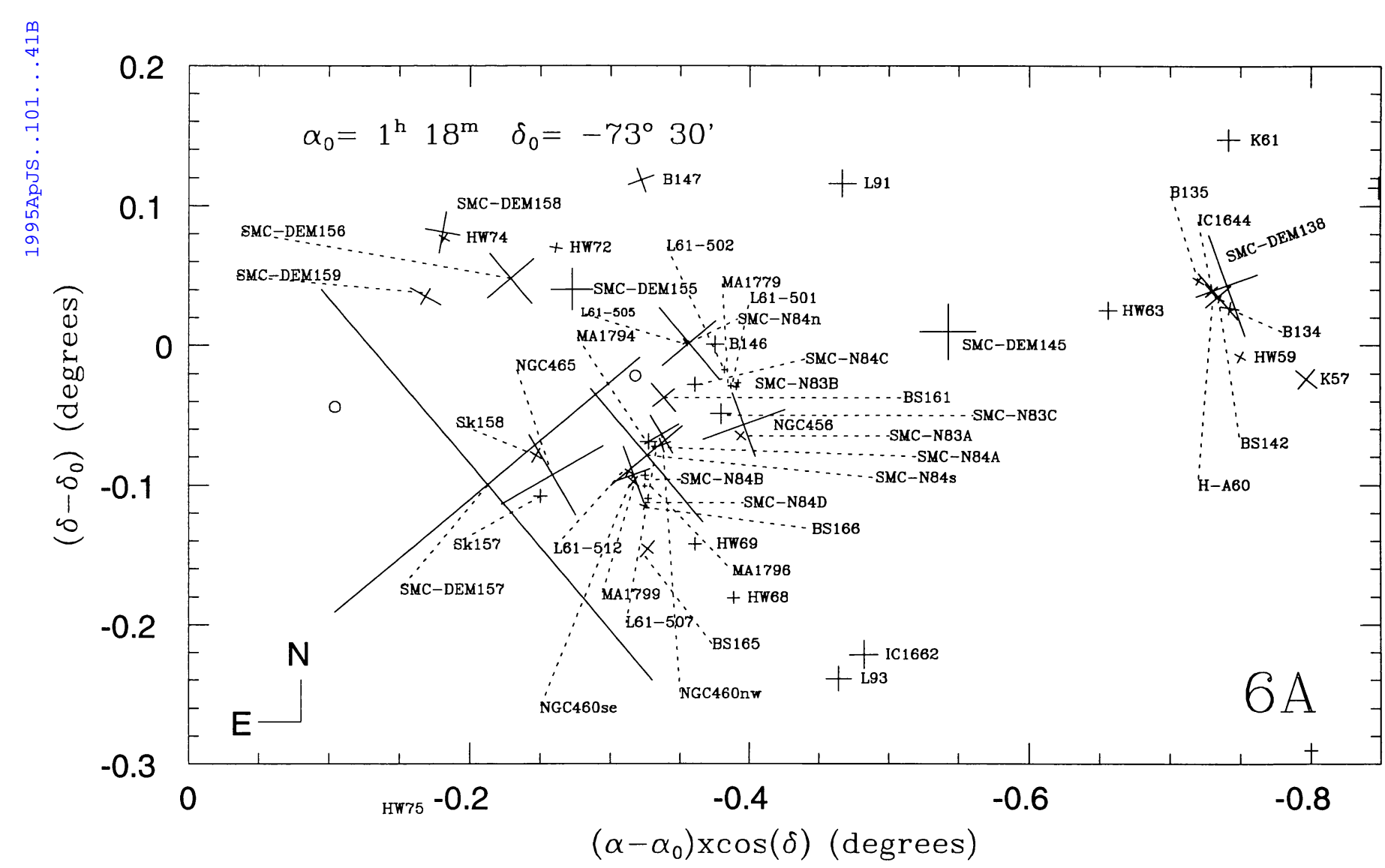

FIG. $2 l$ 


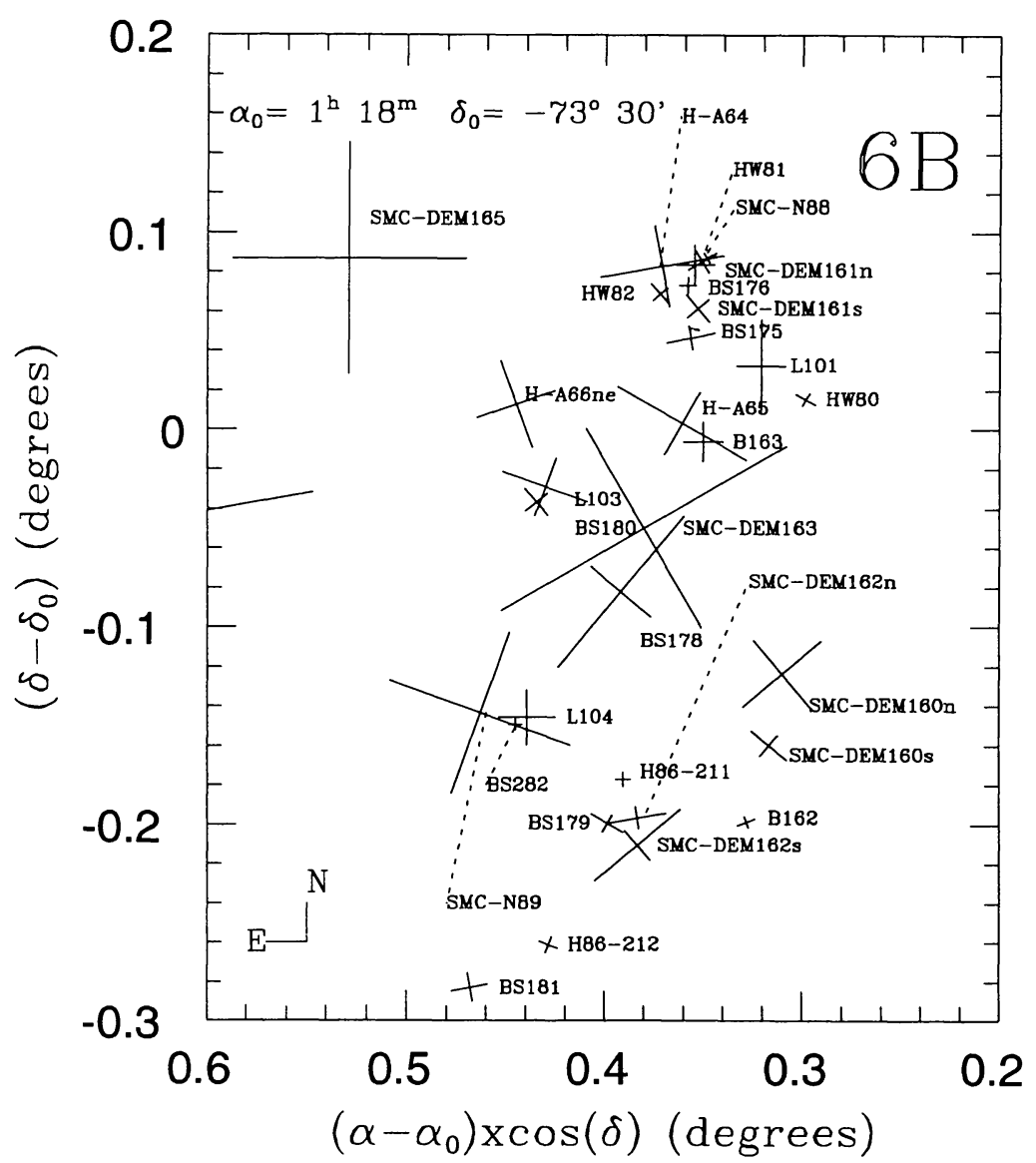

FIG. $2 m$ 


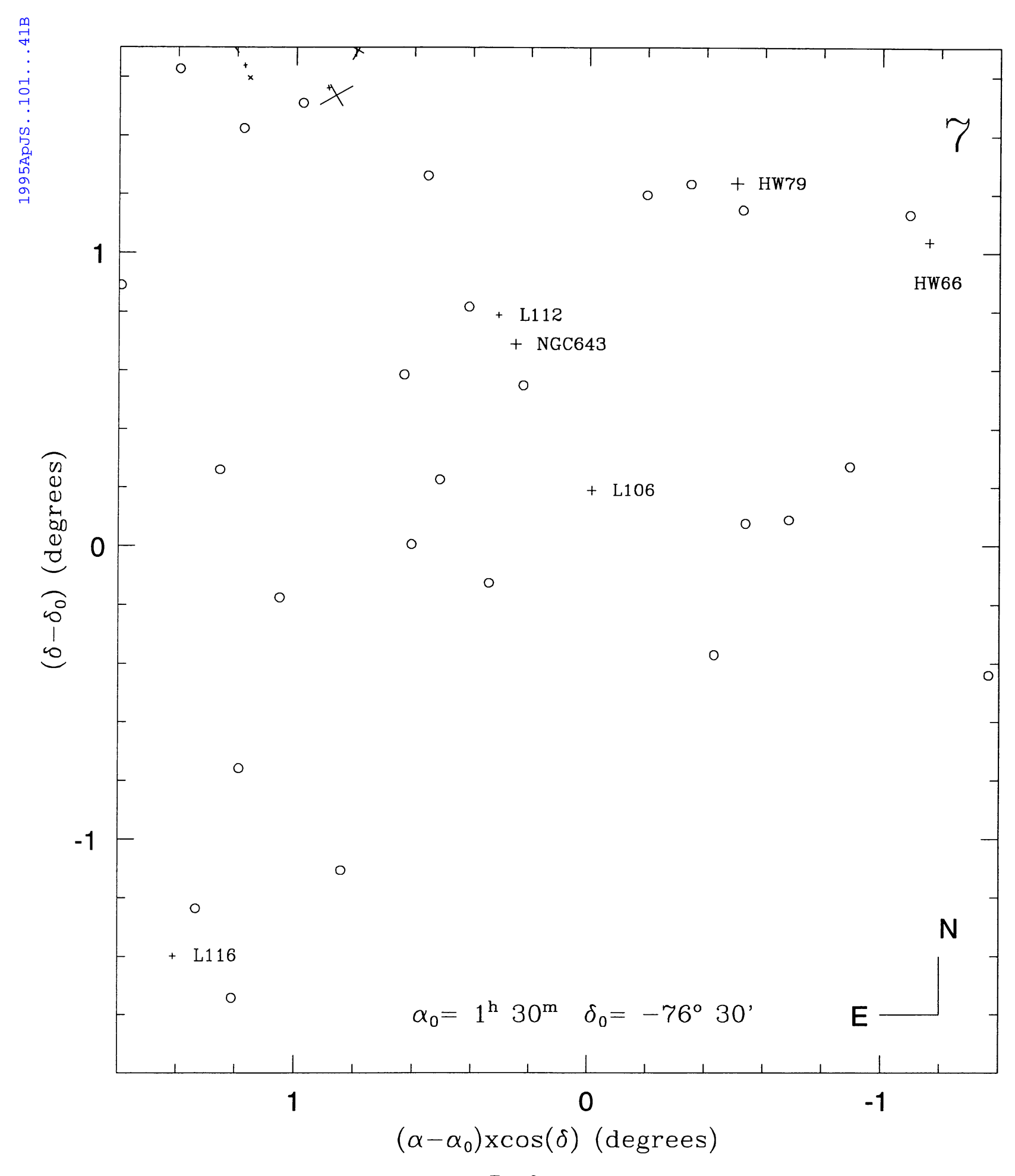

FIG. $2 n$ 


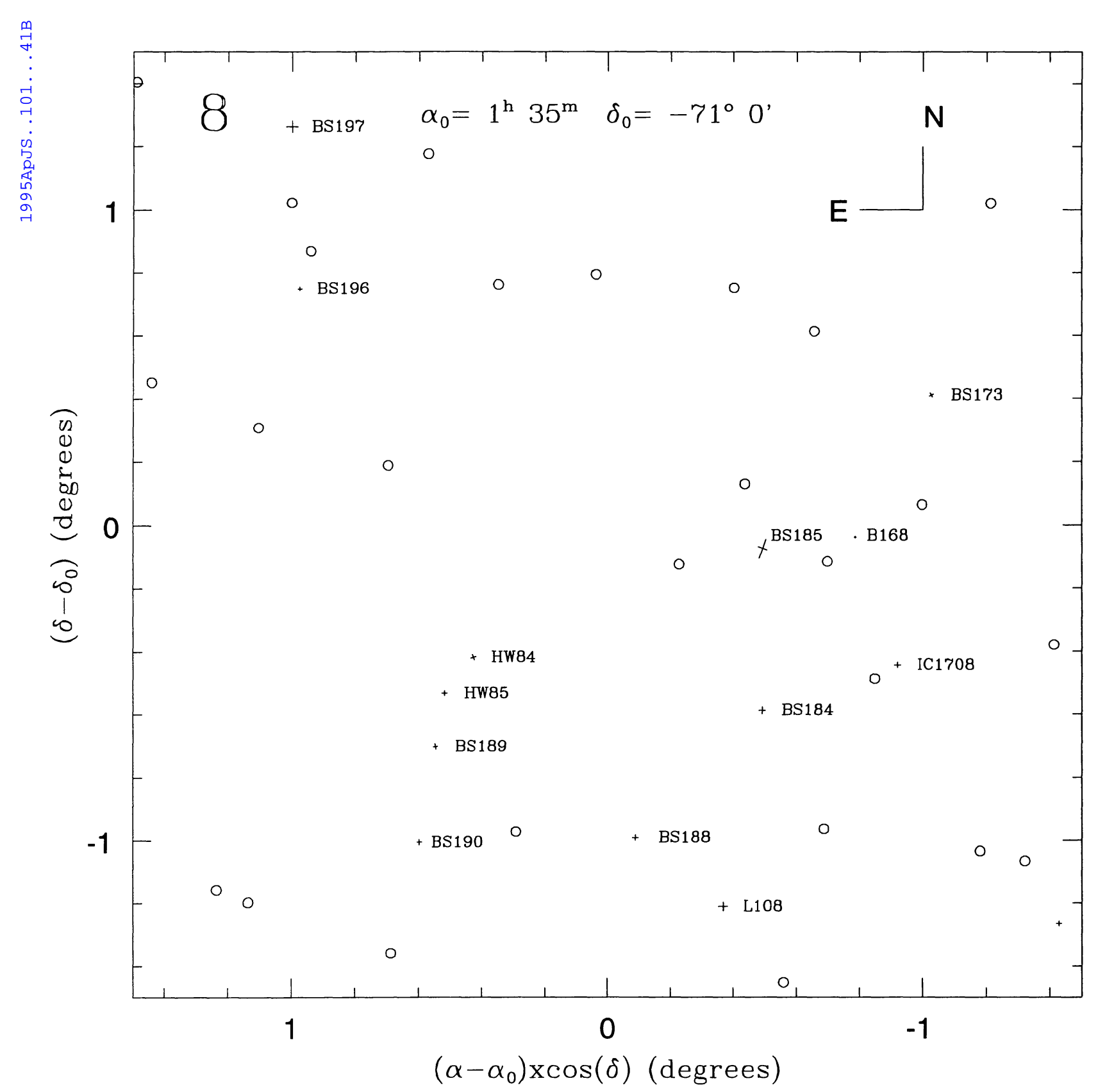

FIG. 20 


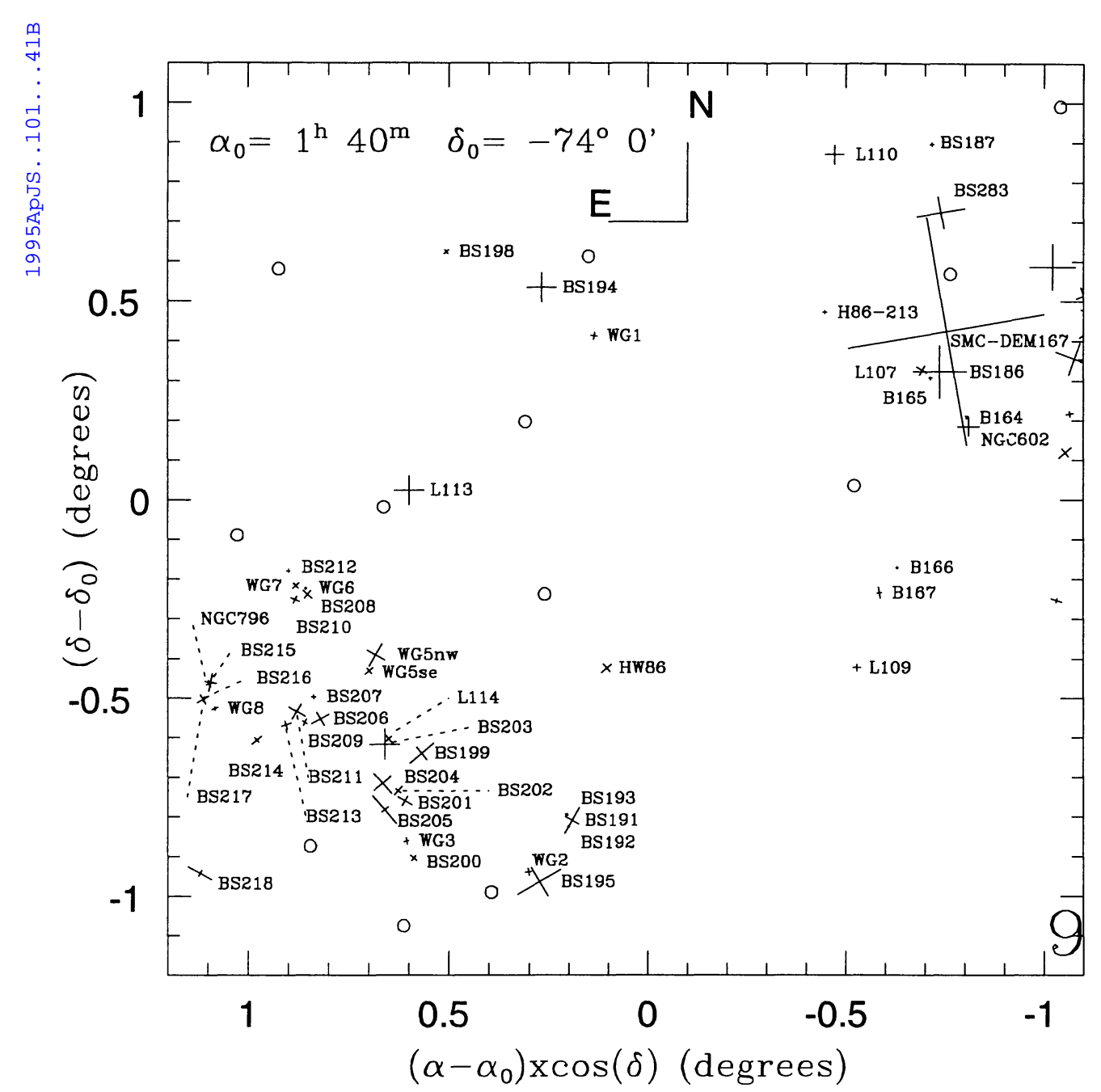

FIG. $2 p$ 


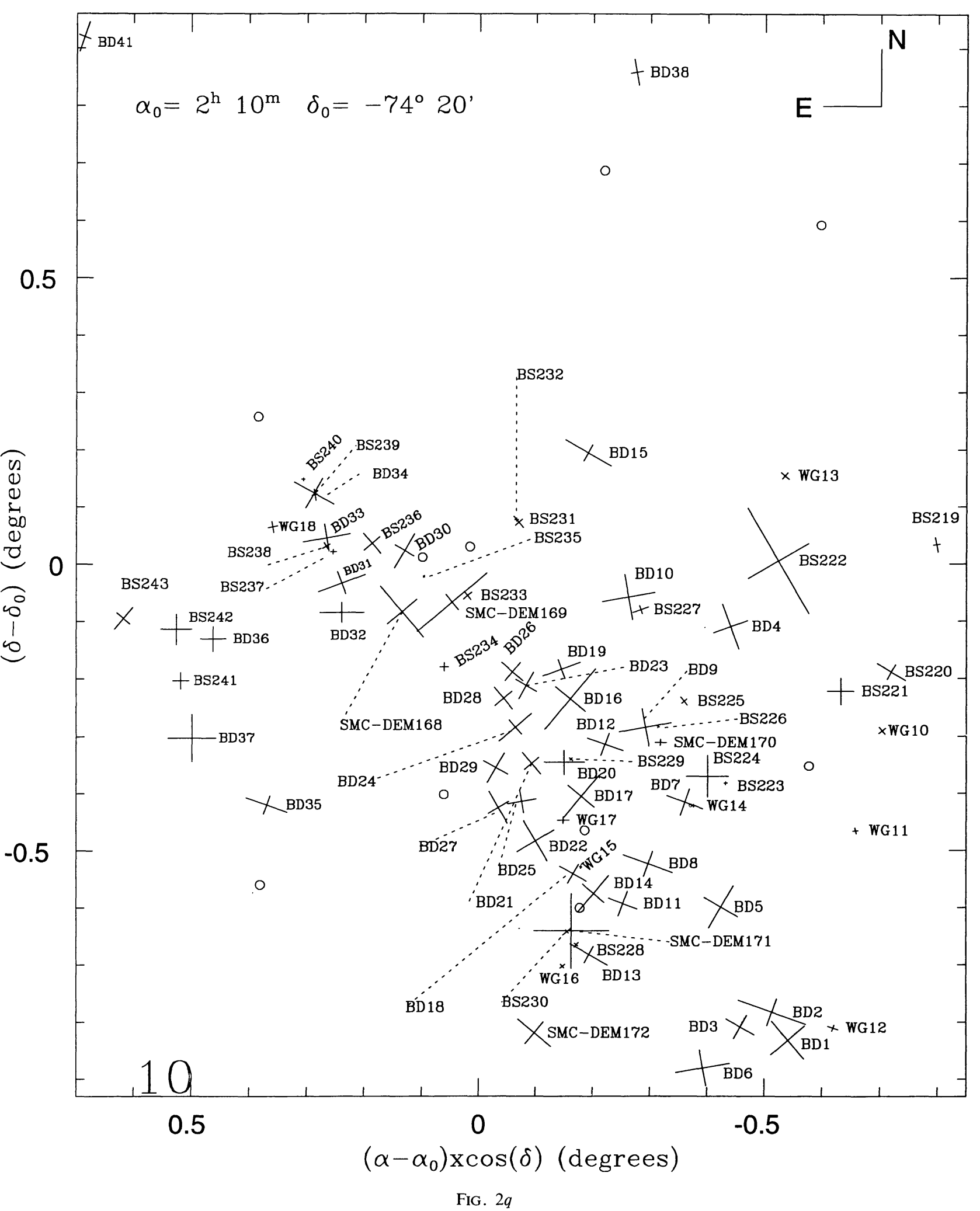




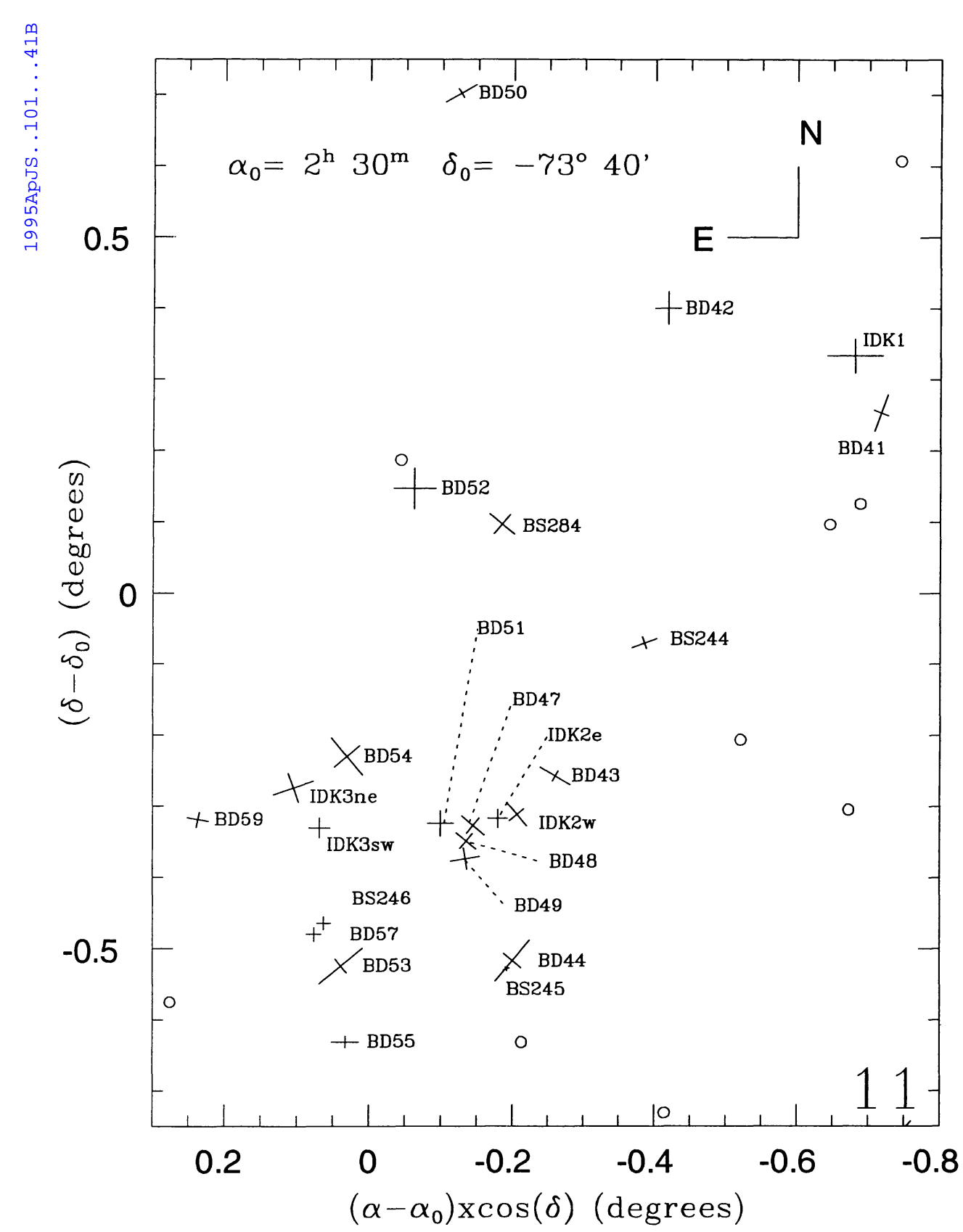

FIG. $2 r$ 


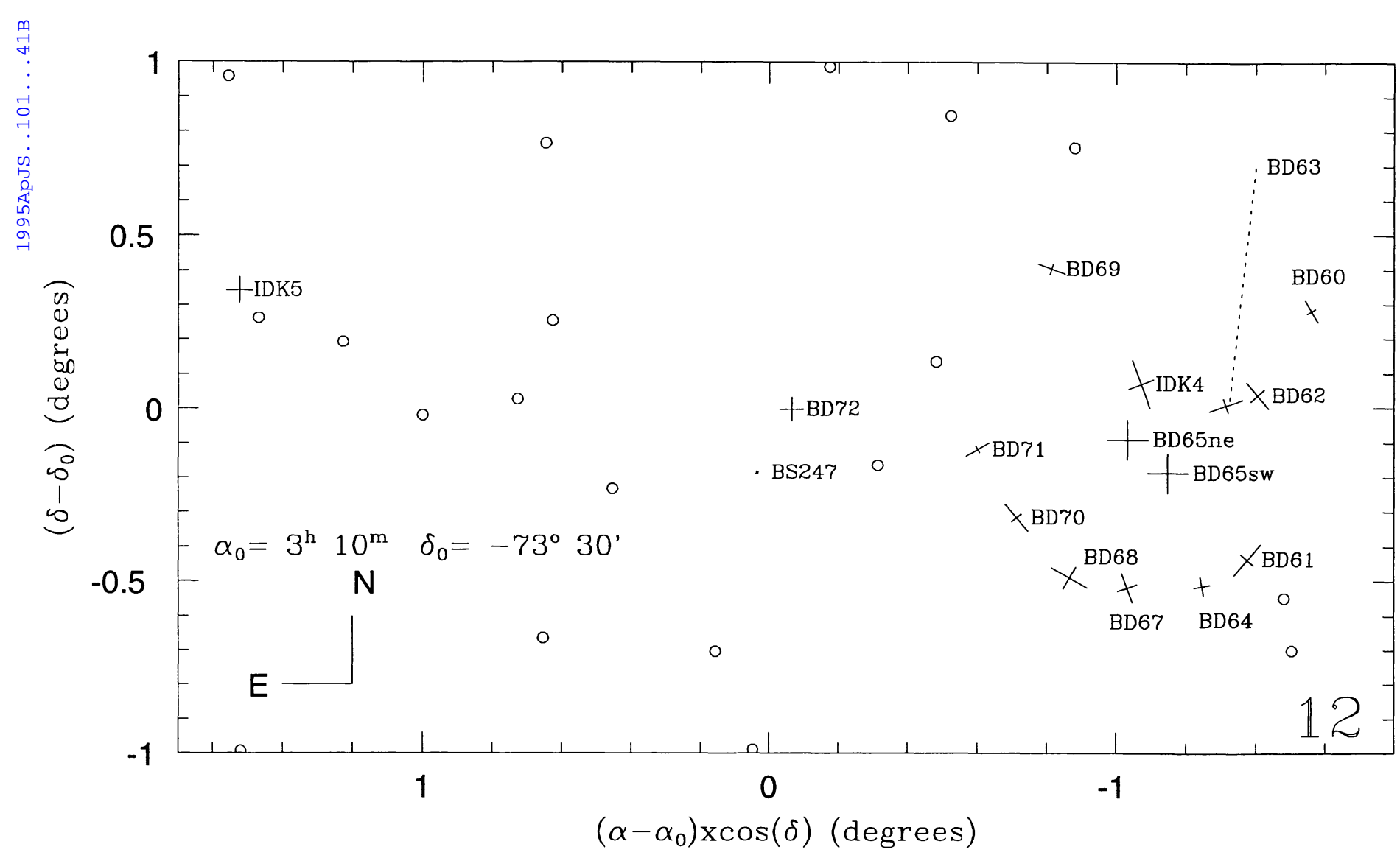

FIG. $2 s$
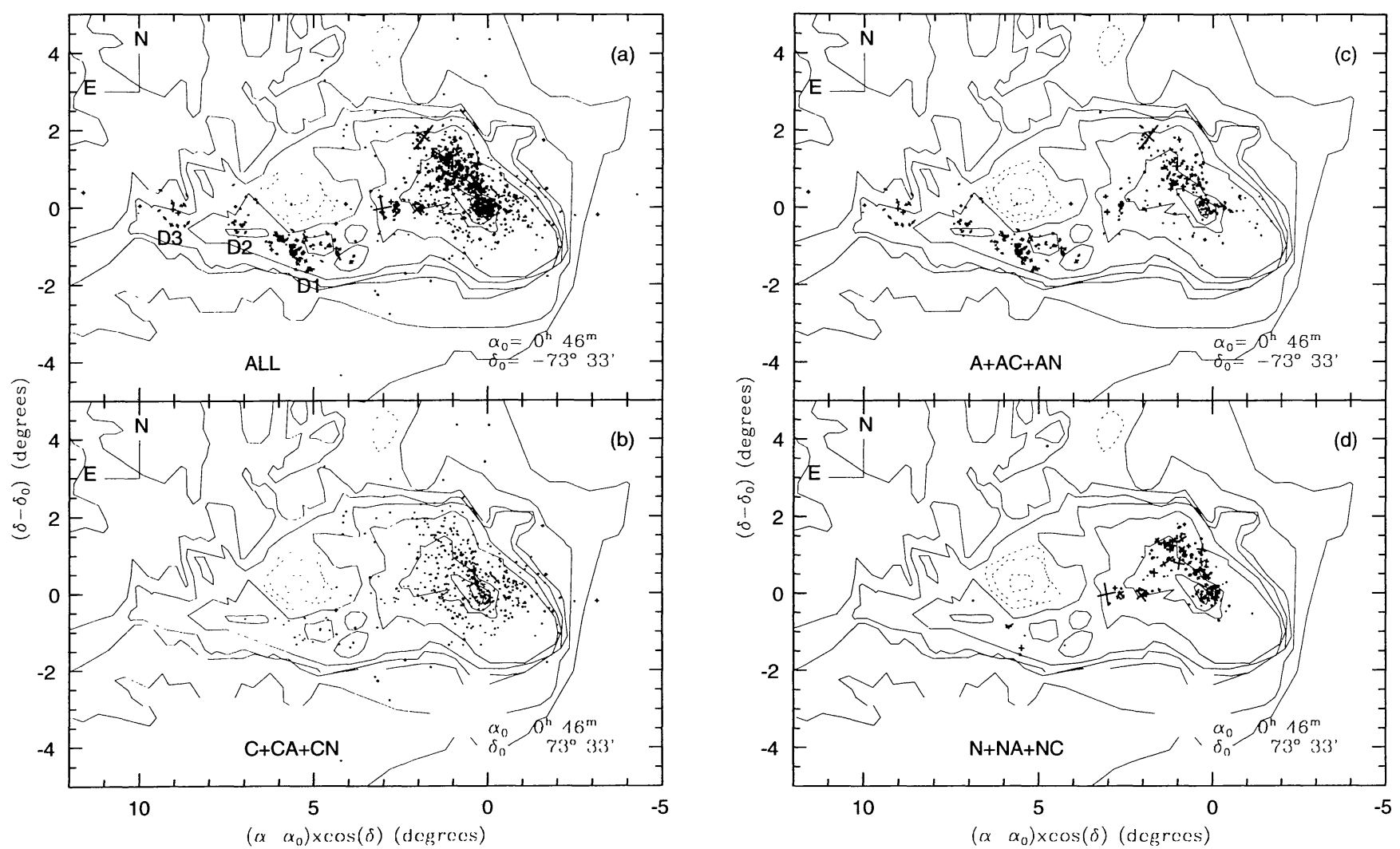

FIG. 3.-Spatial distributions of $(a)$ all objects; $(b)$ star clusters; $(c)$ emission-free associations; $(d)$ objects with emission. H I surface density contours from Mathewson \& Ford (1984) for 5, 20, 40, 50, 75, 125, 400,600, and $800 \times 10^{19}$ atoms $\mathrm{cm}^{-2}$ are shown; dashed lines indicate decreasing gradient. Clumps D1, D2, and D3 are candidates for newborn dwarf galaxies $(\S 4)$. 
two moderately populous blue clusters (NGC 796 and L114) which are still embedded in their stellar associations, as well as several small clusters (Fig. 3b), whereas D2 and D3 do not have any cluster. Fornax has four large globular clusters, another which is looser and fainter, and a concentration of stars which might be a disrupting cluster (Hodge 1961), whereas Sculptor, Draco, and Ursa Minor do not have any cluster. Integrated $U B V$ photometry (van den Bergh 1981, and references therein ) gives $V=11.18,(B-V)=0.42$ and $(\mathrm{U}-\mathrm{B})=$ -0.12 for L114 in a 62" diaphragm; for NGC $796 V=13.68$, but the diaphragm ( 32 ") is too small as compared to the cluster size (Table 2 ), and only the color $(B-V)=-0.06$ is available. These $U B V$ colors imply an equivalent SWB III type for L114 and consequently an age $\approx 100 \mathrm{Myr}$ (Bica, Clariá, \& Dottori $1992)$, whereas the $(B-V)$ color of NGC 796 is compatible with an SWB $0-$ I type (age $<30 \mathrm{Myr})$. An SWB III cluster fades $\Delta V=3-5 \mathrm{mag}$ as it evolves to a $10 \mathrm{Gyr}$ or older globular cluster, depending on the adopted initial mass function slope in the models (see, e.g., Girardi \& Bica 1993). Combining this to the SMC distance modulus and foreground reddening, we estimate for L114 $M_{V} \approx-4.0 \pm 1.5$. This is comparable to Fornax 1, the faintest (probably disrupting) cluster in Fornax, whereas the other five Fornax clusters are considerably brighter $\left(-8.2<M_{V}<-6.5\right.$; Webbink 1985). We conclude that the D1 clump may show up a faint cluster when it will have evolved to $\approx 10 \mathrm{Gyr}$, if a compact cluster like L114 survives to the dynamical evaporation of stars (Wielen 1988).

D1 is currently forming stars by the presence of several SMC-DEM objects from Meaburn (1986); D2 has BS244, which seems to be an emission nebula on the R and $\mathrm{J}$ films, whereas D3 has no emission object. We may be witnessing the birth of dwarf spheroidal galaxies from ejected debris of the LMC/SMC interaction. The D1 clump, which is relatively more massive than D2 and D3, might end up with multıple stellar generations. Detailed velocity studies would be important to check whether these clumps will be ejected from the Magellanic System or not. If they are indeed newborn dwarf spheroidals, they would be unique close-by laboratories to study star formation processes, and by comparison with present-day dwarf spheroidals to understand how the internal dynamical evolution occurs.

Collisions between disk galaxies may create and eject dwarf galaxies (e.g., Schweizer 1978), and recently a dwarf irregular galaxy was detected at the tip of a tidal tail in NGC 4038/39 (Mirabel, Dottori, \& Lutz 1992). Most of the satellite dwarf spheroidals of the Milky Way lie close to the Magellanic Plane and may be remnants of a Greater Magellanic Galaxy (Kunkel 1979, and references therein). Interacting disks can produce bridges or antennae, and numerical simulations have reproduced such features depending on the disk properties and on the encounter parameters (Barnes \& Hernquist 1992, and references therein). Whether the Magellanic System contains a bridge or a pair of tails is a fundamental question for the tidal model calculations. A counter tidal tail candidate might be the NE stellar population and H I extensions of the SMC. The NE part of the SMC is well populated in terms of star clusters and contains the association BS185; these objects are superimposed on a protuberance of the $\mathrm{H}$ I contours $\sim 4^{\circ}-5^{\circ} \mathrm{NE}$ of the SMC center (Figs. $3 a-3 c$ ). The emission object BS197 and the star cluster BS196 lie $\sim 1^{\circ}$ further away in regions of $\mathrm{H}$ I surface density of $40-50 \times 10^{19}$ atoms cm $\mathrm{cm}^{-2}$ (Figs. $3 a$ and $3 d$ ). These $\mathrm{H}$ I clouds extend $\sim 2^{\circ}$ further north out of our Figure 3 and can be clearly seen in Figure 2 of Mathewson \& Ford (1984).

We show in Figures $4 a-4 c$ the size distributions (mean of major and minor axes in Table 2) for the star clusters $(\mathrm{C}+\mathrm{CN}+\mathrm{CA})$, the emissionless associations $(\mathrm{A}+\mathrm{AN}+\mathrm{AC})$, and the emission nebulae $(\mathrm{NA}+\mathrm{NC}+\mathrm{N})$, respectively. The largest star clusters have $D \sim 5^{\prime}$, and their number increases strongly toward smaller sizes down to $D \sim 0.4$, where detection limit effects become important. The histogram for the emissionless associations shows an abrupt decrease toward the larger diameters at $D \sim 5^{\prime}$, which coincides with the upper limit for star clusters. A few associations exceed this limit, but they might be a superimposition of smaller ones or belong to a different object class, the star clouds. An example is BS153 $\left(47^{\prime} \times 20^{\prime}\right)$, which seems to be detached from the main body of the SMC and contains many star clusters ( see chart 5 in Figs. $2 j$ and $1 a$ ). The size histogram for the associations in the interCloud region $\left(2^{\mathrm{h}}<\alpha<3^{\mathrm{h}} 40^{\mathrm{m}}\right)$ is shown as dashed lines in Figure $4 b$, resulting similar to that of the SMC associations.

A large number of small associations $(D \leq 1.6)$ is present in Figure $4 b$. Some were previously included in star cluster catalogs: (i) Hodge (1986) indicates the association nature of H86-72 and H86-195; (ii) some of Brück's ( 1976) type 4 clusters which are described "as large loose groups mainly blue" are small associations (e.g., B62); (iii) some Westerlund \&

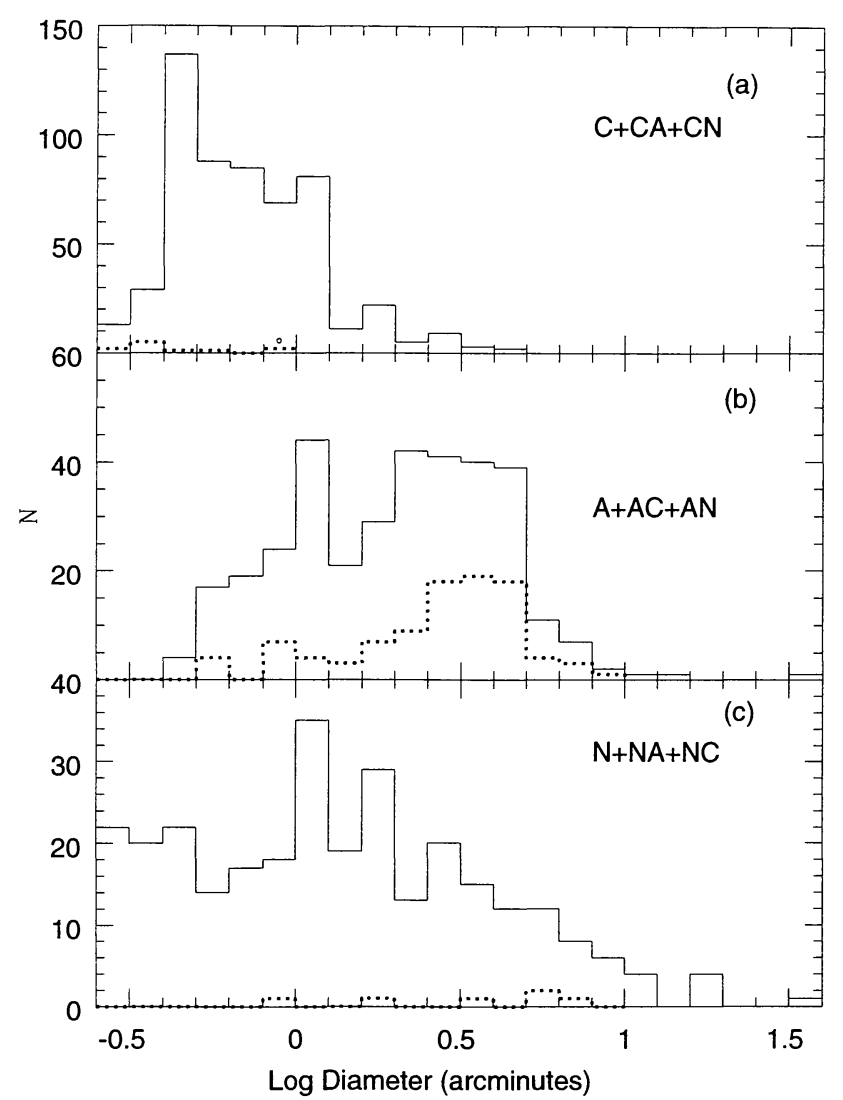

FIG. 4.-Size distributions of $(a)$ star clusters; $(b)$ emissionless associations; $(c)$ objects with emission. Dashed histogram corresponds to objects in the Bridge $\left(2^{\mathrm{h}}<\alpha<3^{\mathrm{h}} 40^{\mathrm{m}}\right)$. 
Glaspey (1971) objects are also small associations (e.g., WG 12 and WG 18). The size range of the small associations $\left(0.5 \leq D \leq 1{ }^{\prime} \cdot 6\right)$ has a comparable number of objects for the corresponding size range in the emission nebula histogram (most are $\mathrm{H}$ II regions with their embedded associations). On the ESO/SERC films, the stellar component of many small NA objects resembles that of small emissionless associations, and some also have designations in Brück's (1976) and Hodge's (1986) catalogs, such as the stellar components of SMC-N58 (B 85) and SMC-N57 (H86-162). The total size range of the objects related to emission nebulae (Fig. $4 c$ ) is comparable to that of the emissionless associations (Fig. 4b), but the histogram shows a smoother decay for larger sizes and it is much more populated for $D<0.5$. The latter effect is caused by the high completeness degree of compact nebulae in the catalogs based on objective prism spectra.

\section{CONCLUSIONS AND PROSPECTIVE WORK}

We have unified the catalogs of star clusters, associations, and emission nebulae in the SMC and in the inter-Cloud region. In the course of this revision we have detected 284 new objects, totaling 1188 entries in the general catalog. The H II regions and their embedded stellar associations of similar size are considered as equivalent entries. We provide accurate positions, cross-identifications, homogeneously measured sizes, and position angles for all the objects. The hierarchical relationship of neighboring objects and the occurrence of cluster pairs and triplets are indicated. The spatial distributions of the associations and emission nebulae are basically encompassed by the $50 \times 10^{19}$ atoms $\mathrm{cm}^{-2} \mathrm{H}$ I surface density contour. Two clumps of extended objects in the Bridge and one at the SMC Wing tip may be newborn dwarf spheroidal galaxies if they are not gravitationally bound to the Clouds. They might represent the early internal dynamical and stellar population evolutionary stages of present-day dwarf spheroidals. In a forthcoming paper the results for the LMC will be presented which, when combined with the present catalog, will make possible an analysis of the overall spatial distribution of nonstellar objects and $\mathrm{H} \mathrm{I}$ in the Magellanic System.

We thank Thaisa Storchi-Bergmann and Miriani Pastoriza for their efforts to acquire the ESO/SERC R and J Atlases to the Instituto de Física, Horacio Dottori for the magnifying lens that we used in the measurements, and Carlos Dutra for creating the computer files for the $\mathrm{H}$ I contours.
Barnes, J. E., \& Hernquist, L. 1992, ARA\&A, 30, 705

Battinelli, P. 1991, A\&A, 244, 69

Battinelli, P., \& Demers, S. 1992, AJ, 104, 1458

Bhatia, R. K., \& MacGillivray, H. T. 1989, A\&A, 211, 9

Bica, E., Clariá, J. J., \& Dottori, H. 1992, AJ, 103, 1859

Bica, E., Clariá, J. J., Dottori, H., Santos, J. F. C., Jr., \& Piatti, A. E. 1995, ApJS, in press

Brück, M. 1976, Occas. Rep. R. Obs. Edinburgh, No. 1

Chu, Y. H., \& Kennicut, R. C., Jr. 1988, AJ, 96, 1874

Copetti, M. V., Pastoriza, M. G., \& Dottori, H. 1985, A\&A, 152, 427

Davies, R. D., Elliot, K. H., \& Meaburn, J. 1976, MmRAS, 81, 89

Eskridge, P. B. 1988, AJ, 96, 1352

Girardi, L., \& Bica, E. 1993, A\&A, 274, 279

Grondin, L., Demers, S., \& Kunkel, W. E. 1992, AJ, 103, 1234

Hatzidimitriou, D., \& Bhatia, R. K. 1990, A\&A, 230, 11

Henize, K. G. 1956, ApJS, 2, 315

Heydari-Malayeri, M., Magain, P., \& Remy, M. 1989, A\&A, 222, 41

Hodge, P. W. 1961, AJ, 66, 83

. 1971, ARA\&A, 9, 35

1985, PASP, 97, 530

1986, PASP, 98, 1113

Hodge, P. W., \& Wright, F. W. 1967, The Large Magellanic Cloud (Washington, DC: Smithsonian Press) . 1974, AJ, 79, 858

Press)

1977, The Small Magellanic Cloud (Seattle: Univ. Washington

Irwin, M. J. 1991, in IAU Symp. 148, The Magellanic Clouds, ed. R. Haynes \& D. Milne (Dordrecht: Kluwer), 453

Irwin, M. J., Demers, S., \& Kunkel, W. E. 1990, AJ, 99, 191

Kontizas, M., Morgan, D. H., Hatzidimitriou, D., \& Kontizas, E. 1990, A\&AS, 84, 527

Kron, G. E. 1956, PASP, 68,125

Kunkel, W. E. 1979, ApJ, 228, 718

\section{REFERENCES}

Lake, G. 1990, MNRAS, 244, 701

Lauberts, A. 1982, The ESO/Uppsala Survey of the ESO(B) Atlas (Garching: European Southern Observatory)

Lindsay, E. M. 1958, MNRAS, 118, 172

. 1961, AJ, 66, 169

Madore, B. F., \& Arp, H. C. 1979, ApJ, 227, L103

Mateo, M., Olszewski, E., Welch, D. L., Fischer, P., \& Kunkel, W. 1991, AJ, 102, 914

Mathewson, D. S., \& Ford, V. L. 1984, IAU Symp. 108, Structure and Evolution of the Magellanic Clouds, ed. S. van den Bergh \& K. S. de Boer (Dordrecht: Reidel), 125

Mathewson, D. S., Ford, V. L., Dopita, M. A., \& Tuohy, I. R. 1984, ApJS, 55,189

Mathewson, D. S., Ford, V. L., Dopita, M. A., Tuohy, I. R., Long, K. S., \& Helfand, D. J. 1983, ApJS, 51, 345

Meaburn, J. 1986, MNRAS, 223, 317

Meyssonnier, N., \& Azzopardi, M. 1993, A\&AS, 102, 451

Mirabel, I. F., Dottori, H., \& Lutz, D. 1992, A\&A, 256, L19

Olszewski, E. W., Harris, H. C., Schommer, R. A., \& Canterna, R. W. 1988, AJ, 95,84

Paltoglou, G., \& Freeman, K. C. 1987, in Structure and Dynamics of Elliptical Galaxies, ed. T. de Zeeuw (Dordrecht: Reidel), 447

Pryor, C., \& Kormendy, J. 1990, AJ, 100, 127

Sanduleak, N. 1968, AJ, 73, 246

Schweizer, F. 1978, in Structure and Properties of Nearby Galaxies, ed. E. M. Berkhuijsen \& R. Wielebinski (Dordrecht: Reidel), 279

van den Bergh, S. 1981, A\&AS, 46, 79

Webbink, R. F. 1985, in Dynamics of Star Clusters, ed. J. Goodman \& P. Hut (Dordrecht: Reidel), 541

Welch, D. L. 1991, AJ, 101, 538

Westerlund, B. E., \& Glaspey, J. 1971, A\&A, 10, 1

Wielen, R. 1988, in Globular Cluster Systems in Galaxies, ed. J. E. Grindlay \& A. G. Davis Philip (Dordrecht: Reidel), 393 